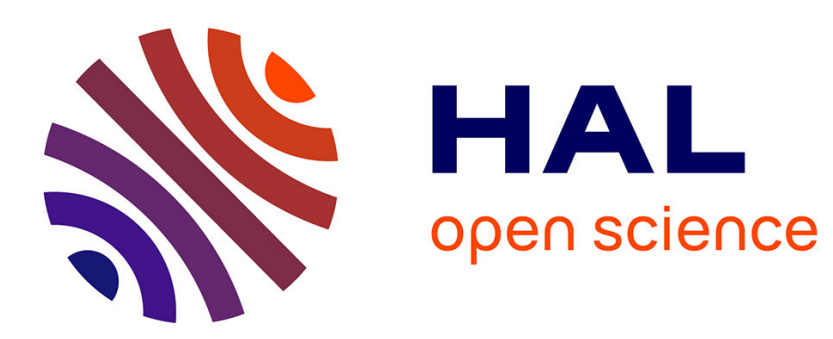

\title{
Error Estimates for the implicit MAC scheme for the compressible Navier-Stokes equations
}

\author{
Thierry Gallouët, David Maltese, Antonin Novotny
}

\section{To cite this version:}

Thierry Gallouët, David Maltese, Antonin Novotny. Error Estimates for the implicit MAC scheme for the compressible Navier-Stokes equations. Numerische Mathematik, 2019, 141 (2), pp.495-567. hal-02078390

\section{HAL Id: hal-02078390 \\ https://hal.science/hal-02078390}

Submitted on 25 Mar 2019

HAL is a multi-disciplinary open access archive for the deposit and dissemination of scientific research documents, whether they are published or not. The documents may come from teaching and research institutions in France or abroad, or from public or private research centers.
L'archive ouverte pluridisciplinaire HAL, est destinée au dépôt et à la diffusion de documents scientifiques de niveau recherche, publiés ou non, émanant des établissements d'enseignement et de recherche français ou étrangers, des laboratoires publics ou privés. 


\title{
Error Estimates for the implicit MAC scheme for the compressible Navier-Stokes equations
}

\author{
Thierry Gallouët David Maltese Antonin Novotny*
}

November 17, 2018

\begin{abstract}
Aix-Marseille Université, CNRS, Centrale Marseille, I2M, UMR 7373, 13453 Marseille, France
and
\end{abstract}

IMATH, EA 2134, Université de Toulon BP 20132, 83957 La Garde, France

\begin{abstract}
We prove existence of a solution to the implicit MAC scheme for the compressible Navier-Stokes equations. We derive error estimates for this scheme on two and three dimensional Cartesian grids. Error estimates are obtained by using the discrete version of the relative energy method introduced on the continuous level in [15]. A systematic use of the theoretical "continuous" analysis of the equations in combination with the numerical tools is crucial for the result. This error estimate does not uses stability hypotheses on the solution of the numerical scheme.
\end{abstract}

Keywords: Compressible fluids, Navier-Stokes equations, Cartesian grids, Marker-And-Cell scheme, Error estimates.

AMS classification 35Q30, 65N12, 76N10, 76N15, 76M12, 76M20

\section{Introduction}

The aim of this paper is to derive error estimates for approximate solutions of the compressible barotropic Navier-Stokes equations obtained by the Marker-And-Cell scheme (MAC scheme in short). These equations are posed on the time-space domain $Q_{T}=(0, T) \times \Omega$, where $\Omega$ is a bounded domain of $\mathbb{R}^{d}, d=2,3$, adapted to the MAC scheme (see Section 3 ), and $T>0$, and read:

$$
\begin{aligned}
& \partial_{t} \varrho+\operatorname{div}(\varrho \boldsymbol{u})=0, \\
& \partial_{t}(\varrho \boldsymbol{u})+\operatorname{div}(\varrho \boldsymbol{u} \otimes \boldsymbol{u})+\nabla p(\varrho)=\mu \Delta \boldsymbol{u}+(\mu+\lambda) \nabla \operatorname{div} \boldsymbol{u},
\end{aligned}
$$

supplemented with the initial conditions

$$
\varrho(0, \boldsymbol{x})=\varrho_{0}(\boldsymbol{x}), \varrho \boldsymbol{u}(0, \boldsymbol{x})=\varrho_{0} \boldsymbol{u}_{0},
$$

where $\varrho_{0}$ and $\boldsymbol{u}_{0}$ are given functions from $\Omega$ to $\mathbb{R}_{+}^{*}$ and $\mathbb{R}^{d}$ respectively, and boundary conditions

$$
\boldsymbol{u}_{\mid(0, T) \times \partial \Omega}=0 .
$$

In the above equations, the unknown functions are the scalar density field $\varrho(t, \boldsymbol{x}) \geq 0$ and vector velocity field $\boldsymbol{u}=\left(u_{1}, \ldots, u_{d}\right)(t, \boldsymbol{x})$, where $t \in(0, T)$ denotes the time and $\boldsymbol{x} \in \Omega$ is the space variable. The viscosity coefficients $\mu$ and $\lambda$, assumed to be constant, are such that

$$
\mu>0, \lambda+\mu \geq 0
$$

${ }^{*}$ This work was supported by the MODTERCOM project within the APEX programme of the Provence-Alpes-Côte d'Azur region 
Notice that hypothesis (1.4) covers the natural physical condtions

$$
\mu>0, \lambda+\frac{2}{3} \mu \geq 0
$$

In the compressible barotropic Navier-Stokes equations, the pressure is a given function of the density. Here we assume that the pressure satisfies

$$
\begin{gathered}
p \in C([0, \infty)), p \in C^{2}(0, \infty), p(0)=0, p^{\prime}(\varrho)>0 \text { for all } \varrho>0, \\
\lim _{\varrho \rightarrow \infty} \frac{p^{\prime}(\varrho)}{\varrho^{\gamma-1}}=p_{\infty}>0, \inf _{\varrho \in(0,1)} \frac{p^{\prime}(\varrho)}{\varrho}=p_{0}>0
\end{gathered}
$$

where $\gamma>1$. We notice that assumptions (1.5) are compatible with the isentropic pressure law $p(\varrho)=\varrho^{\gamma}$ provided $1<\gamma \leq 2$.

The main underlying idea of this paper is to derive the error estimates for approximate solutions of problem (1.1)-(1.5) obtained by time and space discretization by using the discrete version of the relative energy method introduced for equations (1.1a), (1.1b) on the continuous level in $[15,18,19]$.

The theoretical analysis of system (1.1-1.3) is quite recent (see e.g. [40] for the existence and uniqueness of strong solutions on a short time interval, [37], [17], [13] [38] for the existence of weak solutions on a large time interval, [15], [18], [19] for the stability theory and weak strong uniqueness). In contrast with the incompressible Navier-Stokes equations (where the above results can be achieved by classical tools and are quite robust with respect to various perturbations), especially the last two results mentioned above, cannot be obtained by standard arguments. Instead, they essentially rely on several recent tools of analysis (as e.g. compensated compactness or renormalization techniques for the transport equation) tailored to the particular structure of the system. In this analysis the minor deviation in the structure may discredit the result.

We are confronted with the similar difficulties on the level of the convergence analysis of the numerical schemes disretizing system (1.1-1.3). Indeed, if the structure of the discretization does not reproduce faithfully enough the structure of equations on the continuous level, the convergence proofs or error estimates are likely to fail. Strangely enough, there does not seem to be an apparent correlation between the empirical computational efficiency of a numerical scheme and availability of a proof of convergence or an error estimate. There are apparently computationally effcient discretizations without available convergence proofs and inefficient (slowly convergent schemes) with available convergence proofs.

The investigation of convergence properties of discrete solutions to numerical schemes for system (1.11.3) has a short history. The academic scheme (based on finite vomume/finite element discretization on primal tetrahedral meshes) suggested in [36] became a precursor of such studies: 1) Convergence of discrete solutions of this scheme to weak solutions has been proved in [36] for large values of $\gamma(\gamma>3)$. 2) Convergence to measure-valued solutions for small values of $\gamma(1<\gamma \leq 2)$ has been shown in [16]. 3) Finally, error estimates with respect to a strong solution has been obtained in [24]. In spite of its good theoretical properties the discretization method [36] (and its finite-difference counterpart [31]) is ill conditioned and inefficient in the practical numerical simulations.

The staggered schemes (based on finite volume/finite element or finite volume/finite-difference discretization with the velocity defined on a dual mesh - see e.g. [7], [12], [20], [21], [30] for the CrouzeixRaviart type velocity discretization on tetrahedral meshes (CR), [39], [30] for the Ranacher-Turek type velocity discretization on quadrilateral or hexagonal meshes (RT), and [25], [26], [30] with the velocity defined on a dual mesh in the context of final differences on "box" meshes (so called Marker And Cell (MAC) discretization) - provide clearly much better experimental computational efficiency and speed. Although the rigorous mathematical formulation of the staggered schemes (and notably of the MAC scheme) is at first glance lengthy and more involved than the formulation of similar disretizations using only primal mesh, their experimental speed of convergence is worth the effort and investment. Among them, the MAC scheme seems to be more efficient than CR and RT for several reasons: 1) The velocity discretization requires less variables, 2) The discretization of convective terms appears in numerical experiments to be more stable compared to RT and CR, 3) It is particularly efficient for the simulations of flows having dominated direction. Its only disadvantage in many industrial applications is its 'box' mesh which does not allow to approximate effitiently general domains. In many situations, however, its advantages prevail the drawback of the quadrilateral mesh, and it is therefore at the basis of several 
semi-industrial or industrial codes for computing compressible flows. We quote e.g. the code $\mathrm{CALIF}^{3} \mathrm{D}$ of the French Institute of Nuclear Security (IRSN), see [3], to name at least one example. The MAC scheme is also largely used by the scientific community for verifying physical experiments whenever they can be performed in the geometry on cubes (as e.g. direct simulations of high Reynolds number flows, testing of large scale models, simulations of complex flows as e.g. two-phase flows or combustion).

In spite of their computational efficiency, the staggered schemes for the fully nonlinear system (1.1)(1.3) so far resist to any attempts of any type of convergence proofs. This paper is intended to fill this gap at least for the issue of the unconditional error estimates for a large class of staggered schemes interesting from the point of view of mathematical simulations.

The discrete relative energy method was suggested in [24] in the context of the academic finitevolume/finite element scheme proposed in [36] on primal tetrahedral mesh. The method provides unconditional estimate of error between any numerical solution of the scheme [36] and a classical solution of equations (1.1)-(1.5), without any additional assumption on the numerical solution. This is highly wanted result, first of its type in the mathematical literature. The natural question arises whether a similar method can lead to similar unconditional error estimates for the less academic and more practical numerical schemes, notably the staggered schemes.

The main goal of this paper is to get unconditional error estimates for the MAC scheme implicit in time (although its 'box mesh' composed of parallelepipeds can be seen quite restrictive from the point of view of applications). It is to be noticed here that the same techniques - but less involved - can be applied to other staggered schemes that remove the disadvantage of the 'box mesh'. This is the case of e.g. the lowest degree Crouzeix-Raviart finite element discretization of velocity on simplical meshes or the Ranacher-Turek finite elements. We explain great lines of this issue and formulate the final result in Appendix B letting the details of proofs to the interested reader. It is worth to mention that the proofs for these schemes are more simple at many places. At this point, the consultation of paper [30] - where the authors develop an uniform formalism for a broad class including all staggered schemes mentioned above - would be of big help.

In spite of the fact that we stuck to the [24] methodology, the proofs remain still difficult. Not speaking on technicalities linked to the approximations, interpolations and projections to the relevant function spaces related to the MAC discretization, the most involved part is the treatment of transport terms in the continuity and momentum equations which requires derivation of quite sophisticated formulas involving primal and dual fluxes, see Lemma 3.6. In this part, our approach is reminiscent to the recent work [30] devoted to the staggered space approximations to the Euler equations.

Since the very beginning of the introduction of the Marker-and-Cell (MAC) scheme [29], it is claimed that this discretization is suitable for both incompressible and compressible flow problems (see [27, 28] for the seminal papers, $[2,5,6,32,33,35,41-43,45,46]$ for subsequent developments and [47] for a review). The use of the MAC scheme in the incompressible case is now standard, and the proof of convergence for the MAC scheme in primitive variables has been recently been completed [23].

The paper is organized as follows. After recalling the fundamental setting of the problem and the relative energy inequality in the continuous case in Section 2, we proceed in Section 3 to the discretization: we introduce the discrete meshes and functional spaces and the definition of the numerical scheme, and state a known existence result, along with the main result of the paper, that is the error estimate, which is stated in Theorem 3.2. The remaining sections are devoted to the proof of Theorem 3.2:

- In Section 4 we derive estimates provided by the scheme.

- In Section 5, we derive the discrete intrinsic version of the relative energy inequality for the solutions of the numerical scheme (see Theorem 5.1). We then transform this inequality to a more convenient form, see Lemma 5.1.

- Finally, in Section 6, we investigate the form of the discrete relative energy inequality with the test functions being strong solutions to the original problem. This investigation is formulated in Lemma 6.1 and finally leads to a Gronwall type estimate formulated in Lemma 7.1. The latter yields the error estimates and finishes the proof of the main result.

The Theorem 3.2 remains valid for other finite volume schemes with staggered space discretization as e.g. non conforming Rannacher-Turek finite elements or the lowest degree Crouzeix-Raviart finite 
elements on simplical meshes. These results are formulated without proofs, that are similar and simpler than those for the MAC scheme, in Appendix B, Theorem B.1.

\section{The continuous problem}

The aim of this section is to recall some fundamental notions and results for the continuous problem. We begin by the definition of weak solutions to problem (1.1)- (1.5). Let us introduce the Helmholtz's function defined by

$$
\mathscr{H}(\varrho)=\varrho \int_{1}^{\varrho} \frac{p(t)}{t^{2}} \mathrm{~d} t, \varrho \geq 0 .
$$

Note that $\mathscr{H} \in C\left(\mathbb{R}_{+}\right), \mathscr{H}(1)=0$ and that $\mathscr{H}$ is a solution on $\mathbb{R}_{+}^{\star}$ of the ordinary differential equation

$$
\varrho \mathscr{H}^{\prime}-\mathscr{H}=p
$$

with the constant of integration fixed such that $\mathscr{H}(1)=0$. Note also that

$$
\mathscr{H}^{\prime \prime}(\varrho)=\frac{p^{\prime}(\varrho)}{\varrho} .
$$

Definition 2.1 (Weak solutions). Let $\varrho_{0}: \Omega \rightarrow(0,+\infty)$ and $\boldsymbol{u}_{0}: \Omega \rightarrow \mathbb{R}^{d}$ with finite energy $\mathscr{E}_{0}=$ $\int_{\Omega}\left(\frac{1}{2} \varrho_{0}\left|\boldsymbol{u}_{0}\right|^{2}+\mathscr{H}\left(\varrho_{0}\right)\right) \mathrm{d} \boldsymbol{x}$ and finite mass $0<M_{0}=\int_{\Omega} \varrho_{0} \mathrm{~d} \boldsymbol{x}$. We shall say that the pair $(\varrho, \boldsymbol{u})$ is a weak solution to the problem (1.1)-(1.5) emanating from the initial data $\left(\varrho_{0}, \boldsymbol{u}_{0}\right)$ if:

1. $\varrho \in L^{\infty}\left(0, T ; L^{\gamma}(\Omega)\right)$, $\varrho \geq 0$ a.e. in $(0, T) \times \Omega, \boldsymbol{u} \in L^{2}\left(0, T ; H_{0}^{1}(\Omega)^{d}\right)$ and $\varrho|\boldsymbol{u}|^{2} \in L^{\infty}\left(0, T ; L^{1}(\Omega)\right)$.

2. The continuity equation (1.1a) is satisfied in the following weak sense

$$
\int_{0}^{T} \int_{\Omega}\left(\varrho \partial_{t} \varphi+\varrho \boldsymbol{u} \cdot \nabla \varphi\right) \mathrm{d} \boldsymbol{x} \mathrm{dt}=-\int_{\Omega} \varrho_{0} \varphi(0, \boldsymbol{x}) \mathrm{d} \boldsymbol{x}
$$

for any $\varphi \in C_{c}^{\infty}([0, T] \times \bar{\Omega})$ such that $\varphi(T, \cdot)=0$.

3. The momentum equation (1.1b) is satisfied in the weak sense,

$$
\begin{aligned}
\int_{0}^{T} \int_{\Omega}\left(\varrho \boldsymbol{u} \cdot \partial_{t} \boldsymbol{\psi}+\varrho \boldsymbol{u} \otimes \boldsymbol{u}: \nabla \boldsymbol{\psi}+p(\varrho) \operatorname{div} \boldsymbol{\psi}\right) \mathrm{d} \boldsymbol{x} \mathrm{dt} \\
\quad-\int_{0}^{T} \int_{\Omega}(\mu \nabla \boldsymbol{u}: \nabla \boldsymbol{\psi} \mathrm{d} \boldsymbol{x} \mathrm{dt}+(\mu+\lambda) \operatorname{div} \boldsymbol{u} \operatorname{div} \boldsymbol{\psi}) \mathrm{d} \boldsymbol{x} \mathrm{dt}=-\int_{\Omega} \varrho_{0} \boldsymbol{u}_{0} \cdot \boldsymbol{\psi}(0, \boldsymbol{x}) \mathrm{d} \boldsymbol{x}
\end{aligned}
$$

for any $\boldsymbol{\psi} \in C_{c}^{\infty}([0, T] \times \Omega)^{d}$ such that $\boldsymbol{\psi}(T, \cdot)=\mathbf{0}$.

4. The following energy inequality is satisfied a.e in $(0, T)$

$$
\int_{\Omega}\left(\frac{1}{2} \varrho|\boldsymbol{u}|^{2}+\mathscr{H}(\varrho)\right)(\tau) \mathrm{d} \boldsymbol{x}+\int_{0}^{\tau} \int_{\Omega}\left(\mu|\nabla \boldsymbol{u}|^{2}+(\mu+\lambda)|\operatorname{div} \boldsymbol{u}|^{2}\right) \mathrm{d} \boldsymbol{x} \mathrm{dt} \leq \mathscr{E}_{0} .
$$

Note that the existence of weak solutions emanating from the finite energy initial data is well-known on bounded Lipschitz domains under assumptions (1.4) and (1.5) provided $\gamma>d /(d-1)$, see Lions [37] for "large" values of $\gamma$, Feireisl and coauthors [17] for $\gamma>d /(d-1)$. More details about this problem are avaible in monographs [37], [13], [38].

Remark 1. The density $\varrho$ satisfies the conservation of mass that is

$$
\int_{\Omega} \varrho(t) \mathrm{d} \boldsymbol{x}=M_{0} \text { a.e in }(0, T) .
$$


Let us now introduce the notion of relative energy. We first introduce the function

$$
\begin{aligned}
E: \quad & {[0, \infty) \times(0, \infty) \rightarrow \mathbb{R}, } \\
& (\varrho, r) \mapsto E(\varrho \mid r)=\mathscr{H}(\varrho)-\mathscr{H}^{\prime}(r)(\varrho-r)-\mathscr{H}(r),
\end{aligned}
$$

where $\mathscr{H}$ is defined by (2.1). Since $p$ satisfies the thermodynamic stability condition that is

$$
p^{\prime}>0 \text { on } \mathbb{R}_{+}^{*},
$$

the function $\mathscr{H}$ is strictly convex on $\mathbb{R}_{+}^{\star}$ and we have

$$
E(\varrho \mid r) \geq 0 \text { and } E(\varrho \mid r)=0 \Leftrightarrow \varrho=r .
$$

More precisely, $E$ satisfies the following algebraic inequality whose straightforward proof is left to the reader:

Lemma 2.1. Let $0<a<b<\infty$. Then there exists a number $c=c\left(\gamma, p_{\infty}, a, b, \min _{[a, b]} p, \min _{[a / 2,2 b]} p^{\prime}\right)>$ 0 such that for all $\varrho \in[0, \infty)$ and $r \in[a, b]$

$$
\begin{aligned}
E(\varrho \mid r) \geq c(a, b)\left(\varrho^{\gamma} 1_{\left.\mathbb{R}_{+} \backslash a / 2,2 b\right]}(\varrho)+1_{\mathbb{R}_{+} \backslash[a / 2,2 b]}(\varrho)+(\varrho-r)^{2} 1_{[a / 2,2 b]}(\varrho)\right) \\
\geq c(a, b, \gamma)\left(|\varrho-r|^{\gamma} 1_{\mathbb{R}_{+} \backslash[a / 2,2 b]}(\varrho)+(\varrho-r)^{2} 1_{[a / 2,2 b]}(\varrho)\right) .
\end{aligned}
$$

In order to measure a "distance" between a weak solution $(\varrho, \boldsymbol{u})$ of the compressible Navier-Stokes system and any other state $(r, \boldsymbol{U})$ of the fluid, we introduce the relative energy functional, defined by

$$
\mathfrak{E}(\varrho, \boldsymbol{u} \mid r, \boldsymbol{U})=\int_{\Omega}\left(\frac{1}{2} \varrho|\boldsymbol{u}-\boldsymbol{U}|^{2}+E(\varrho \mid r)\right) \mathrm{d} \boldsymbol{x} .
$$

It was proved recently in [15] that, provided assumption (1.5) holds, any weak solution satisfies the following so-called relative energy inequality

$$
\begin{aligned}
\mathfrak{E}(\varrho, \boldsymbol{u} \mid r, \boldsymbol{U})(\tau)-\mathfrak{E}(\varrho, & \boldsymbol{u} \mid r, \boldsymbol{U})(0) \\
& +\int_{0}^{\tau} \int_{\Omega}\left(\mu|\nabla(\boldsymbol{u}-\boldsymbol{U})|^{2}+(\mu+\lambda)|\operatorname{div}(\boldsymbol{u}-\boldsymbol{U})|^{2}\right) \mathrm{d} \boldsymbol{x} \mathrm{dt} \\
& \leq \int_{0}^{\tau} \int_{\Omega}(\mu \nabla \boldsymbol{U}: \nabla(\boldsymbol{U}-\boldsymbol{u})+(\mu+\lambda) \operatorname{div} \boldsymbol{U} \operatorname{div}(\boldsymbol{U}-\boldsymbol{u})) \mathrm{d} \boldsymbol{x} \mathrm{dt} \\
& +\int_{0}^{\tau} \int_{\Omega} \varrho \partial_{t} \boldsymbol{U} \cdot(\boldsymbol{U}-\boldsymbol{u}) \mathrm{d} \boldsymbol{x} \mathrm{dt}+\int_{0}^{\tau} \int_{\Omega} \varrho \boldsymbol{u} \cdot \nabla \boldsymbol{U} \cdot(\boldsymbol{U}-\boldsymbol{u}) \mathrm{d} \boldsymbol{x} \mathrm{dt} \\
& +\int_{0}^{\tau} \int_{\Omega} \frac{r-\varrho}{r} p^{\prime}(r) \partial_{t} r \mathrm{~d} \boldsymbol{x} \mathrm{dt}-\int_{0}^{\tau} \int_{\Omega} \frac{\varrho}{r} p^{\prime}(r) \nabla r \cdot \boldsymbol{u} \mathrm{d} \boldsymbol{x} \mathrm{dt} \\
& -\int_{0}^{\tau} \int_{\Omega} p(\varrho) \operatorname{div} \boldsymbol{U} \mathrm{d} \boldsymbol{x} \mathrm{dt} .
\end{aligned}
$$

for a.a. $\tau \in(0, T)$, and for any pair of test functions

$$
r \in C^{1}([0, T] \times \bar{\Omega}), r>0, \boldsymbol{U} \in C^{1}([0, T] \times \bar{\Omega})^{3},\left.\boldsymbol{U}\right|_{\partial \Omega}=\mathbf{0} .
$$

Moreover if $(r, \boldsymbol{U})$ is a sufficiently strong solution to problem (1.1)-(1.5) emanating from initial data $\left(r_{0}, \boldsymbol{U}_{0}\right)$, the right member becomes quadratic in difference $(\varrho-r, \boldsymbol{u}-\boldsymbol{U})$ and inequality $(2.11)$ reduces

$$
\begin{aligned}
\mathfrak{E}(\varrho, \boldsymbol{u} \mid r, \boldsymbol{U})(\tau)+\int_{0}^{\tau} \int_{\Omega}\left(\mu|\nabla(\boldsymbol{u}-\boldsymbol{U})|^{2}+(\mu+\lambda) \mid\right. & \left.\left.\operatorname{div}(\boldsymbol{u}-\boldsymbol{U})\right|^{2}\right) \mathrm{d} \boldsymbol{x} \mathrm{dt} \\
& \leq \mathfrak{E}\left(\varrho_{0}, \boldsymbol{u}_{0} \mid r(0), \boldsymbol{U}(0)\right)+\int_{0}^{\tau} \mathscr{R}(\varrho, \boldsymbol{u} \mid r, \boldsymbol{U}) \mathrm{dt}
\end{aligned}
$$


where

$$
\begin{aligned}
\mathscr{R}(\varrho, \boldsymbol{u} \mid r, \boldsymbol{U})=\int_{\Omega} & (\varrho-r)\left(\partial_{t} \boldsymbol{U}+\boldsymbol{U} \cdot \nabla \boldsymbol{U}\right) \cdot(\boldsymbol{U}-\boldsymbol{u}) \mathrm{d} \boldsymbol{x}+\int_{\Omega} \varrho(\boldsymbol{u}-\boldsymbol{U}) \cdot \nabla \boldsymbol{U} \cdot(\boldsymbol{U}-\boldsymbol{u}) \mathrm{d} \boldsymbol{x} \\
& +\int_{\Omega} \frac{\nabla p(r)}{r}(r-\varrho) \cdot(\boldsymbol{u}-\boldsymbol{U}) \mathrm{d} \boldsymbol{x}-\int_{\Omega}\left(p(\varrho)-p^{\prime}(r)(\varrho-r)-p(r)\right) \operatorname{div} \boldsymbol{U} \mathrm{d} \boldsymbol{x} .
\end{aligned}
$$

In order to obtain a stability result of strong solutions in the class of weak solutions, the goal is to find an estimate of the left hand side of from below by (2.12) by

$$
c \int_{0}^{\tau}\|\boldsymbol{u}-\boldsymbol{U}\|_{W^{1,2}(\Omega)^{3}}^{2} \mathrm{~d} \boldsymbol{x}-\bar{c}^{\prime} \int_{0}^{\tau} \mathfrak{E}(\varrho, \boldsymbol{u} \mid r, \boldsymbol{U})(t) \mathrm{dt}+\mathfrak{E}(\varrho, \boldsymbol{u} \mid r, \boldsymbol{U})(\tau)
$$

and thanks to lemma 2.1 the right hand side from above by

$$
\mathfrak{E}\left(\varrho_{0}, \boldsymbol{u}_{0} \mid r(0), \boldsymbol{U}(0)\right)+\delta \int_{0}^{\tau}\|\boldsymbol{u}-\boldsymbol{U}\|_{W^{1,2}(\Omega)^{3}}^{2} \mathrm{~d} \boldsymbol{x}+c^{\prime}(\delta) \int_{0}^{\tau} a(t) \mathfrak{E}(\varrho, \boldsymbol{u} \mid r, \boldsymbol{U})(t) \mathrm{dt}
$$

with any $\delta>0$, where $c>0$ is independent of $\delta, \bar{c}^{\prime} \geq 0, c^{\prime}=c^{\prime}(\delta)>0$ and $a \in L^{1}(0, T)$. This process leads to the estimate

$$
\mathfrak{E}(\varrho, \boldsymbol{u} \mid r, \boldsymbol{U})(\tau) \leq \mathfrak{E}\left(\varrho_{0}, \boldsymbol{u}_{0} \mid r(0), \boldsymbol{U}(0)\right)+c \int_{0}^{\tau} a(t) \mathfrak{E}(\varrho, \boldsymbol{u} \mid r, \boldsymbol{U})(t) \mathrm{dt} .
$$

It remains to conclude by using Gronwall Lemma. This implies the stability of strong solutions in the class of weak solutions. The exact statement is formulated in the following proposition, see [15].

Proposition 2.1 (Estimate on the relative energy). Let $\Omega$ be a bounded Lipschitz domain. Assume that the viscosity coefficients satisfy assumptions (1.4) and that the pressure $p$ satisfy

$$
p \in C([0, \infty)), p \in C^{2}(0, \infty), p(0)=0, p^{\prime}(\varrho)>0 \text { for all } \varrho>0, \lim _{\varrho \rightarrow \infty} \frac{p^{\prime}(\varrho)}{\varrho^{\gamma-1}}=p_{\infty}>0,
$$

where $\gamma>\frac{6}{5}$. Let $(\varrho, \boldsymbol{u})$ be a weak solution to problem (1.1)-(1.5) emanating from initial data $\left(\varrho_{0}>0, \boldsymbol{u}_{0}\right)$, with finite energy $\mathscr{E}_{0}$ and finite mass $M_{0}=\int_{\Omega} \varrho_{0} \mathrm{~d} x>0$. Let $(r, \boldsymbol{U})$ that belongs to the class

$$
\begin{gathered}
0<\underline{r} \leq r \leq \bar{r}, r \in L^{\infty}((0, T) \times \Omega), \\
\boldsymbol{U} \in L^{2}\left(0, T ; H_{0}^{1}(\Omega)^{3}\right), \\
\nabla r, \nabla^{2} \boldsymbol{U} \in L^{2}\left(0, T ; L^{q}(\Omega)\right), q>\max \left(3, \frac{6 \gamma}{5 \gamma-6}\right),
\end{gathered}
$$

be a strong solution of the same equations with initial data $(r(0), \boldsymbol{U}(0))=\left(r_{0}, \boldsymbol{U}_{0}\right)$. Then there exists

$$
c=c\left(T, \Omega, M_{0}, \mathscr{E}_{0}, \underline{r}, \bar{r}, \gamma,\|\operatorname{div} \boldsymbol{U}\|_{L^{1}\left(0, T ; L^{\infty}(\Omega)\right)},\left\|\left(\nabla r, \nabla^{2} \boldsymbol{U}\right)\right\|_{L^{2}\left(0, T ; L^{q}(\Omega)^{12}\right)}\right)>0
$$

such that for almost all $t \in(0, T)$,

$$
\mathfrak{E}(\varrho, \boldsymbol{u} \mid r, \boldsymbol{U})(t) \leq c \mathfrak{E}\left(\varrho_{0}, \boldsymbol{u}_{0} \mid r_{0}, \boldsymbol{U}_{0}\right) .
$$

The goal of the present paper is to obtain estimate of type (2.18) for $(\varrho, \boldsymbol{u})$ being a numerical solution of Problem (1.1)-(1.5) obtained by the MAC discretization.

\section{The numerical scheme}

\subsection{Space discretization}

We assume that the closure of the domain $\Omega$ is a union of closed rectangles $(d=2)$ or closed orthogonal parallelepipeds $(d=3)$ with mutually disjoint interiors, and, without loss of generality, we assume that the edges (or faces) of these rectangles (or parallelepipeds) are orthogonal to the canonical basis vectors, denoted by $\left(\boldsymbol{e}^{(1)}, \ldots, \boldsymbol{e}^{(d)}\right)$, 
Definition 3.1 (MAC grid). A discretization of $\Omega$ with $M A C$ grid, denoted by $\mathscr{D}$, is given by $\mathscr{D}=(\mathscr{M}, \mathscr{E})$, where:

- The density and pressure (or primal) grid denoted by $\mathscr{M}$, consists of a union of possibly non uniform (closed) rectangles ( $d=2)$ or (closed) parallelpipeds $(d=3)$, the edges (or faces) of these rectangles (or parallelepipeds) are orthogonal to the canonical basis vectors; a generic cell of this grid is denoted by $K$ ( a closed set), and its mass center $\boldsymbol{x}_{K}$. It is a conforming grid, meaning that

$$
\bar{\Omega}=\cup_{K \in M} K \text {, where } \operatorname{int}(K) \cap \operatorname{int}(L)=\emptyset \text { whenever }(K, L) \in \mathscr{M}^{2}, K \neq L,
$$

and if $K \cap L \neq \emptyset$ then $K \cap L$ is a common face or edge or vertex of $K$ and $L$. A generic face (or edge in the two-dimensional case) of such a cell is denoted by $\sigma \in \mathscr{E}(K)$ (a closed set), and its mass center $\boldsymbol{x}_{\sigma}$, where $\mathscr{E}(K)$ denotes the set of all faces of $K$. We denote by $\boldsymbol{n}_{\sigma, K}$ the unit normal vector to $\sigma$ outward $K$. The set of all faces of the mesh is denoted by $\mathscr{E}$; we have $\mathscr{E}=\mathscr{E}_{\text {int }} \cup \mathscr{E}_{\text {ext }}$, where $\mathscr{E}_{\text {int }}$ (resp. $\mathscr{E}_{\text {ext }}$ ) are the edges of $\mathscr{E}$ that lie in the interior (resp. on the boundary) of the domain. The set of faces that are orthogonal to the $i^{\text {th }}$ unit vector $\boldsymbol{e}^{(i)}$ of the canonical basis of $\mathbb{R}^{d}$ is denoted by $\mathscr{E}^{(i)}$, for $i=1, \ldots, d$. We then have $\mathscr{E}^{(i)}=\mathscr{E}_{\text {int }}^{(i)} \cup \mathscr{E}_{\text {ext }}^{(i)}$, where $\mathscr{E}_{\text {int }}^{(i)}\left(\right.$ resp. $\mathscr{E}_{\text {ext }}^{(i)}$ ) are the edges of $\mathscr{E}^{(i)}$ that lie in the interior (resp. on the boundary) of the domain. Finally, for $i=1, \ldots, d$ and $K \in \mathscr{M}$, we denote $\mathscr{E}^{(i)}(K)=\mathscr{E}(K) \cap \mathscr{E}^{(i)}$ and $\mathscr{E}_{\text {int }}^{(i)}(K)=\mathscr{E}(K) \cap \mathscr{E}_{\text {int }}^{(i)}$.

- For each $\sigma \in \mathscr{E}$, we write that $\sigma=K \mid L$ if $\sigma=\partial K \cap \partial L$ and we write that $\sigma=\overrightarrow{K \mid L}$ if, furthermore, $\sigma \in \mathscr{E}^{(i)}$ and $\left(\boldsymbol{x}_{L}-\boldsymbol{x}_{K}\right) \cdot \boldsymbol{e}^{(i)}>0$ for some $i \in[1, d] \equiv\{1, \ldots, d\}$. A primal cell $K$ will be denoted $K=\left[\overrightarrow{\sigma \sigma^{\prime}}\right]$ if $\sigma, \sigma^{\prime} \in \mathscr{E}^{(i)} \cap \mathscr{E}(K)$ for some $i=1, \ldots, d$ are such that $\left(\boldsymbol{x}_{\sigma^{\prime}}-\boldsymbol{x}_{\sigma}\right) \cdot \boldsymbol{e}^{(i)}>0$. For a face $\sigma \in \mathscr{E}$, the distance $d_{\sigma}$ is defined by:

$$
d_{\sigma}= \begin{cases}d\left(\boldsymbol{x}_{K}, \boldsymbol{x}_{L}\right) & \text { if } \sigma=K \mid L \in \mathscr{E}_{\mathrm{int}}, \\ d\left(\boldsymbol{x}_{K}, x_{\sigma}\right) & \text { if } \sigma \in \mathscr{E}_{\mathrm{ext}} \cap \mathscr{E}(K)\end{cases}
$$

where $d(\cdot, \cdot)$ denotes the Euclidean distance in $\mathbb{R}^{d}$.

- A dual cell $D_{\sigma}$ associated to a face $\sigma \in \mathscr{E}$ is defined as follows:

* if $\sigma=K \mid L \in \mathscr{C}_{\text {int }}$ then $D_{\sigma}=D_{\sigma, K} \cup D_{\sigma, L}$, where $D_{\sigma, K}$ - a closed set (resp. $D_{\sigma, L}$ - a closed set) is the half-part of $K$ (resp. L) adjacent to $\sigma$ (see Fig. 1 for the two-dimensional case) ;

* if $\sigma \in \mathscr{E}_{\text {ext }}$ is adjacent to the cell $K$, then $D_{\sigma}=D_{\sigma, K}$.

The dual grid $\left\{D_{\sigma}\right\}_{\sigma \in \mathscr{E}(i)}$ of $\Omega$ (sometimes called the $i$-th velocity component grid) verifies for each fixed $i \in\{1, \ldots, d\}$

$$
\bar{\Omega}=\cup_{\sigma \in \mathscr{E}}{ }^{(i)} D_{\sigma}, \quad \operatorname{int}\left(D_{\sigma}\right) \cap \operatorname{int}\left(D_{\sigma^{\prime}}\right)=\emptyset, \sigma, \sigma^{\prime} \in \mathscr{E}^{(i)}, \sigma \neq \sigma^{\prime} .
$$

- A dual face separating two neighboring dual cells $D_{\sigma}$ and $D_{\sigma^{\prime}}$ is denoted by $\epsilon=\sigma \mid \sigma^{\prime}$ or $\epsilon=D_{\sigma} \mid D_{\sigma^{\prime}}$ (a closed set) or $\epsilon=\overrightarrow{\sigma \mid \sigma^{\prime}}$ when specifying its orientation: more precisely we write that $\epsilon=\overrightarrow{\sigma \sigma^{\prime}}$ if $\left(\boldsymbol{x}_{\sigma^{\prime}}-\boldsymbol{x}_{\sigma}\right) \cdot \boldsymbol{e}^{(j)}>0$ for some $j \in[1, d]$. The set of all faces of $D_{\sigma}$ is denoted $\tilde{\mathscr{E}}\left(D_{\sigma}\right)$; it is decomposed to the set of external faces $\tilde{\mathscr{E}}_{\text {ext }}\left(D_{\sigma}\right)=\left\{\epsilon \in \tilde{\mathscr{E}}\left(D_{\sigma}\right) \mid \epsilon \subset \partial \Omega\right\}$ and the set of internal faces $\tilde{\mathscr{E}}_{\text {int }}\left(D_{\sigma}\right)=\left\{\epsilon \in \tilde{\mathscr{E}}\left(D_{\sigma}\right) \mid\right.$ int $\left._{d-1} \epsilon \subset \Omega\right\}$, where int $_{d-1}$ denote the interior in the topology of $\mathbb{R}^{d-1}$. The set of all dual faces of the dual grid $\left\{D_{\sigma}\right\}_{\sigma \in \mathscr{E}(i)}$ is decomposed into the internal and boundary edges: $\widetilde{\mathscr{E}}^{(i)}=\widetilde{\mathscr{E}}_{\text {int }}^{(i)} \cup \widetilde{\mathscr{E}}_{\text {ext }}^{(i)}$, where $\widetilde{\mathscr{E}}_{\text {int }}^{(i)}=\left\{\epsilon=\sigma\left|\sigma^{\prime}\right| \sigma, \sigma^{\prime} \in \mathscr{E}^{(i)}\right\}$ and $\widetilde{\mathscr{E}}_{\text {ext }}^{(i)}=\left\{\epsilon=\partial D_{\sigma} \cap \partial \Omega \mid \sigma \in \mathscr{E}^{(i)}, \partial D_{\sigma} \cap \partial \Omega \neq \emptyset\right\}$. We denote by $\boldsymbol{n}_{\epsilon, D_{\sigma}}$ the unit normal vector to $\epsilon \in D_{\sigma}$ outward $D_{\sigma}$.

We denote for further convenience $\mathbf{n}_{\epsilon}$ and $\mathbf{n}_{\sigma}$ a normal unit vector to face $\epsilon$ and $\sigma$, respectively. We write $\epsilon \perp \sigma$ resp. $\sigma_{\perp} \sigma^{\prime}$ iff $\mathbf{n}_{\epsilon} \cdot \mathbf{n}_{\sigma}=0$ resp. $\mathbf{n}_{\sigma} \cdot \mathbf{n}_{\sigma^{\prime}}=0$. Similarly we write $\epsilon \perp \mathbf{e}^{(j)}$ resp. $\sigma \perp \mathbf{e}^{(j)}$ iff $\mathbf{n}_{\epsilon}$ and $\mathbf{e}^{(j)}$ resp. $\mathbf{n}_{\sigma}$ and $\mathbf{e}^{(j)}$ are parallel. We also denote by $\mathbf{a b}$ the segment $\{\mathbf{a}+t(\mathbf{b}-\mathbf{a}) \mid t \in[0,1]\}$, where $(\mathbf{a}, \mathbf{b}) \in \mathbb{R}^{2 d}$, and by $\boldsymbol{x}_{\epsilon}$ resp. $\boldsymbol{x}_{\sigma \cap \epsilon}$ the mass centers of the face $\epsilon$ resp. of the set $\sigma \cap \epsilon$ (provided it is not empty).

- In order to define bi-dual grid, we introduce the set $\tilde{\mathscr{E}}^{(i, j)}=\left\{\epsilon \in \tilde{\mathscr{E}}^{(i)} \mid \epsilon \perp \boldsymbol{e}^{(j)}\right\}$ of dual faces of the $i$-th component velocity grid that are orthogonal to $\boldsymbol{e}^{(j)}$. A bi-dual cell $D_{\epsilon}$ associated to a face $\epsilon \in \tilde{\mathscr{E}}$ is defined as follows: 
* If $\epsilon=\overrightarrow{\sigma \mid \sigma^{\prime}} \in \tilde{\mathscr{E}}^{(i, j)} \cap \tilde{\mathscr{E}}_{\text {int }}^{(i)}$ then $D_{\epsilon}=\epsilon \times \boldsymbol{x}_{\sigma} \boldsymbol{x}_{\sigma^{\prime}}$ (see Figure 2). (We notice that, if $\sigma, \sigma^{\prime} \in \mathscr{E}^{(i)}$ with $K=\overrightarrow{\left[\sigma \sigma^{\prime}\right]} \in \mathscr{M}$ and $\epsilon=\sigma \mid \sigma^{\prime}$ then $D_{\epsilon}=K$.)

* If $\epsilon \in \tilde{\mathscr{E}}^{(i, j)} \cap \tilde{\mathscr{E}}_{\text {ext }}^{(i)}$ with $\epsilon \in \tilde{\mathscr{E}}\left(D_{\sigma}\right)$ and $i \neq j$ then $D_{\epsilon}=\epsilon \times \boldsymbol{x}_{\sigma} \boldsymbol{x}_{\sigma \cap \epsilon}$.

In the list above we did not consider the sitution $\epsilon \in \tilde{\mathscr{E}}^{(i, i)} \cap \tilde{\mathscr{E}}_{\mathrm{ext}}^{(i)}$ with $\epsilon \in \tilde{\mathscr{E}}\left(D_{\sigma}\right)$. In this case $\epsilon=\sigma \subset \partial \Omega$, and we set for completeness $D_{\epsilon}=\emptyset$.

It is to be noticed that, for each fixed couple $(i, j) \in\{1, \ldots, d\}^{2}$

$$
\cup_{\epsilon \in \tilde{\mathscr{E}}(i, j)} D_{\epsilon}=\bar{\Omega}, \quad \operatorname{int}\left(D_{\epsilon}\right) \cap \operatorname{int}\left(D_{\epsilon^{\prime}}\right)=\emptyset, \epsilon \neq \epsilon^{\prime}, \epsilon, \epsilon^{\prime} \in \tilde{\mathscr{E}}^{(i, j)}
$$

To any dual face $\epsilon \in \tilde{\mathscr{E}}^{(i)}$, we associate a distance $d_{\epsilon}$

$$
d_{\epsilon}=\left\{\begin{array}{c}
d\left(\boldsymbol{x}_{\sigma}, \boldsymbol{x}_{\sigma^{\prime}}\right) \text { if } \epsilon \in \tilde{\mathscr{E}}^{(i, j)} \cap \tilde{\mathscr{E}}_{\text {int }}^{(i)}, \\
d\left(\boldsymbol{x}_{\sigma}, \boldsymbol{x}_{\sigma \cap \epsilon}\right) \text { if } \epsilon \in \tilde{\mathscr{E}}^{(i, j)} \cap \tilde{\mathscr{E}}_{\text {ext }}^{(i)} \text { with } \epsilon \in \tilde{\mathscr{E}}\left(D_{\sigma}\right) \text { and } i \neq j, \\
d_{\sigma} \text { if } \epsilon \in \tilde{\mathscr{E}}^{(i, i)} \cap \tilde{\mathscr{E}}_{\text {ext }}^{(i)} \text { with } \epsilon \in \tilde{\mathscr{E}}\left(D_{\sigma}\right) .
\end{array}\right.
$$

(We notice that the last line in the above definition is irrelevant and pure convention, since in that case $D_{\epsilon}=\emptyset$.)

- We also define the size of the mesh by

$$
h_{\mathscr{M}}=\max \left\{h_{K}, K \in \mathscr{M}\right\}
$$

where $h_{K}$ stands for the diameter of $K$. Moreover if $K=\left[\overrightarrow{\sigma \sigma^{\prime}}\right]$ where $\sigma, \sigma^{\prime} \in \mathscr{E}^{(i)} \cap \mathscr{E}(K)$ for some $i=1, \ldots, d$ we will denote

$$
h_{K}^{(i)}=\frac{|K|}{|\sigma|}=\frac{|K|}{\left|\sigma^{\prime}\right|} .
$$

In the above $|A|$ denotes the $\mathbb{R}^{d}$ - resp. $\mathbb{R}^{d-1}$ - dimensional Lebsgue measure of $A \subset \mathbb{R}^{d}$ resp. $A \subset \mathbb{R}^{d-1}$.

We measure the regularity of the mesh through the positive real number $\eta_{\mathscr{M}}$ defined by

$$
\eta_{\mathscr{M}}=\max \left\{\frac{|\sigma|}{\left|\sigma^{\prime}\right|}, \sigma \in \mathscr{E}^{(i)}, \sigma^{\prime} \in \mathscr{E}^{(j)},(i, j) \in\{1, \ldots, d\}^{2}, i \neq j\right\} .
$$

Finally, we denote by $h_{\sigma}$ the diameter of the face $\sigma \in \mathscr{E}$.

- Some geometric notions introduced in this definition are exposed in the figures 1 and 2:

Definition 3.2 (Discrete spaces). Let $\mathscr{D}=(\mathscr{M}, \mathscr{E})$ be a MAC grid in the sense of Definition 3.1. The discrete density and pressure space $L_{\mathscr{M}}$ is defined as the set of piecewise constant functions over each of the grid cells $K$ of $\mathscr{M}$, and the discrete $i-t h$ velocity component space $H_{\mathscr{C}}^{(i)}$ as the set of piecewise constant functions over each of the grid cells $D_{\sigma}, \sigma \in \mathscr{E}^{(i)}$. As in the continuous case, the Dirichlet boundary conditions (1.3) are (partly) incorporated in the definition of the velocity spaces, and, to this purpose, we introduce $H_{\mathscr{E}, 0}^{(i)} \subset H_{\mathscr{E}}^{(i)}, i=1, \ldots, d$, defined as follows:

$$
H_{\mathscr{E}, 0}^{(i)}=\left\{v \in H_{\mathscr{\mathscr { C }}}^{(i)}, v(\boldsymbol{x})=0 \forall \boldsymbol{x} \in D_{\sigma}, \sigma \in \widetilde{\mathscr{E}}_{\mathrm{ext}}^{(i)}, i=1, \ldots, d\right\} .
$$

We then set $\mathbf{H}_{\mathscr{E}, 0}=\prod_{i=1}^{d} H_{\mathscr{C}, 0}^{(i)}$. Since we are dealing with piecewise constant functions, it is useful to introduce the characteristic functions $\mathscr{X}_{K}, K \in \mathscr{M}$ and $\mathscr{X}_{D_{\sigma}}, \sigma \in \mathscr{E}$ of the density (or pressure) and velocity cells, defined by

$$
\mathscr{X}_{K}(\boldsymbol{x})=\left\{\begin{array}{l}
1 \text { if } \boldsymbol{x} \in K, \\
0 \text { if } \boldsymbol{x} \notin K,
\end{array} \quad \mathscr{X}_{D_{\sigma}}(\boldsymbol{x})=\left\{\begin{array}{l}
1 \text { if } \boldsymbol{x} \in D_{\sigma}, \\
0 \text { if } \boldsymbol{x} \notin D_{\sigma} .
\end{array}\right.\right.
$$

We can then write a function $\boldsymbol{v} \in \mathbf{H}_{\mathscr{C}, 0}$ as $\boldsymbol{v}=\left(v_{1}, \ldots, v_{d}\right)$ with $v_{i}=\sum_{\sigma \in \mathscr{E}_{\text {int }}^{(i)}} v_{\sigma} \mathscr{X}_{D_{\sigma}}, i \in[1, d]$ and a function $q \in L_{\mathscr{M}}$ as $q=\sum_{K \in \mathscr{M}} q_{K} \mathscr{X}_{K}$. 


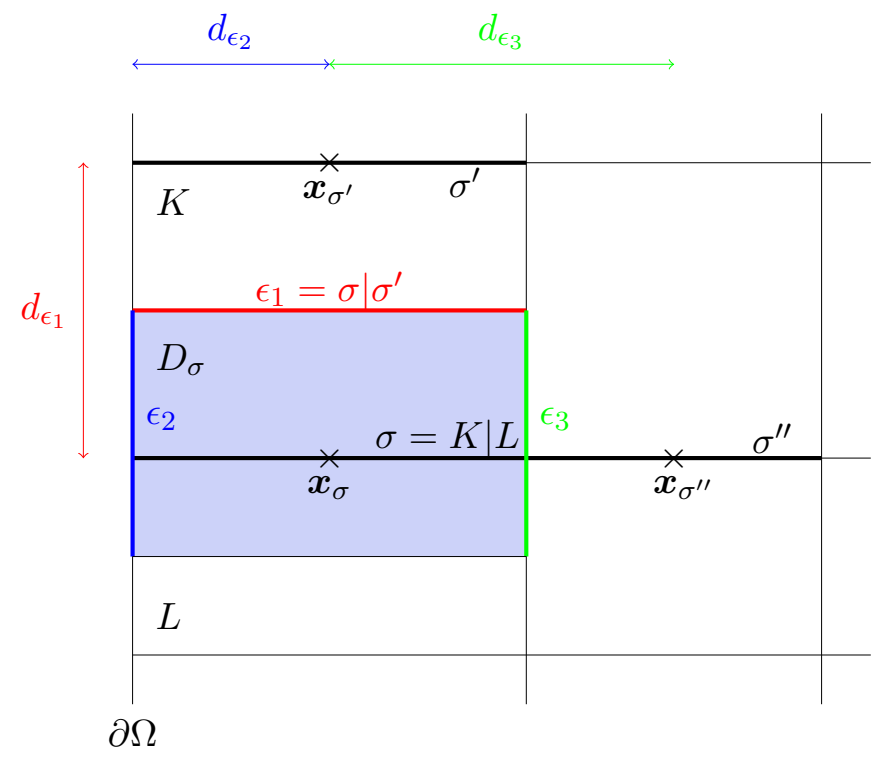

Figure 1: Notations for control volumes and dual cells

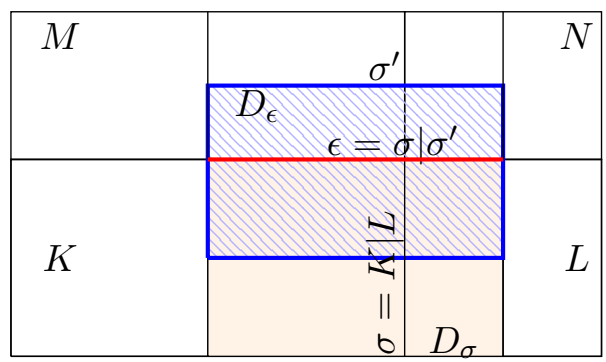

Figure 2: Notations for bi-dual cells

\subsection{Time discretization}

Let us now turn to the time discretization of Problem 1.1. We consider a partition $0=t^{0}<t^{1}<\cdots<$ $t^{N}=T$ of the time interval $(0, T)$, and, for the sake of simplicity, a constant time step $\delta t=t^{n}-t^{n-1}$; hence $t^{n}=n \delta t$ for $n \in\{0, \cdots, N\}$. We denote respectively by $\left\{u_{\sigma}^{n}, \sigma \in \mathscr{E}_{\text {int }}^{(i)}, i \in\{1, \cdots, d\}, n \in\right.$ $\{0, \cdots, N\}\}$, and $\left\{\varrho_{K}^{n}, K \in \mathscr{M}, n \in\{1, \cdots, N\}\right)$ the sets of discrete i-th component of velocity and density unknowns. For $\sigma \in \mathscr{E}_{\text {int }}^{(i)}, i \in\{1, \cdots, d\}$ the value $u_{\sigma}^{n}$ is an expected approximation of the mean value over $\left(t^{n-1}, t^{n}\right) \times D_{\sigma}$ of the i-th component of the velocity of a weak solution, while for $K \in \mathscr{M}$ the value $\varrho_{K}^{n}$ is an expected approximation of the mean value over $\left(t^{n-1}, t^{n}\right) \times K$ of the density of a weak solution. To the discrete unknowns, we associate piecewise constant functions on time intervals and on primal or dual meshes, which are expected approximation of weak solutions, For the velocities, these constant functions are of the form:

$$
u_{i}(t, \boldsymbol{x})=\sum_{n=1}^{N} \sum_{\sigma \in \mathscr{E}_{\mathrm{int}}^{(i)}} u_{\sigma}^{n} \mathscr{X}_{D_{\sigma}}(\boldsymbol{x}) \mathscr{X}_{\left(t^{n-1}, t^{n}\right)}(t),
$$

where $\mathscr{X}_{\left(t^{n-1}, t^{n}\right)}$ is the characteristic function of the interval $\left(t^{n-1}, t^{n}\right)$. We denote by $X_{i, \mathscr{E}, \delta t}$ the set of such piecewise constant functions on time intervals and dual cells, and we set $\boldsymbol{X}_{\mathscr{C}, \delta t}=\prod_{i=1}^{d} X_{i, \mathscr{E}, \delta t}$. For the density, the constant function is of the form:

$$
\varrho(t, \boldsymbol{x})=\varrho_{K}^{n} \text { for } \boldsymbol{x} \in K \text { and } t \in\left(t^{n-1}, t^{n}\right),
$$

and we denote by $Y_{\mathscr{M}, \delta t}$ the space of such piecewise constant functions.

For a given $\boldsymbol{u} \in \boldsymbol{X}_{\mathscr{C}, \delta t}$ associated to the set of discrete velocity unknowns $\left\{u_{\sigma}^{n}, \sigma \in \mathscr{E}_{\text {int }}^{(i)}, i \in\{1, \cdots, d\}, n \in\right.$ $\{1, \cdots, N\}\}$, and for $n \in\{1, \cdots, N\}$, we denote by $u_{i}^{n} \in H_{\mathscr{E}, 0}^{(i)}$ the piecewise constant function defined 
by $u_{i}^{n}(\boldsymbol{x})=u_{\sigma}^{n}$ for $\boldsymbol{x} \in D_{\sigma}, \sigma \in \mathscr{E}_{\text {int }}^{(i)}$, and set $\boldsymbol{u}^{n}=\left(u_{1}^{n}, \ldots, u_{d}^{n}\right)^{t} \in \mathbf{H}_{\mathscr{E}, 0}$. We sometimes write $u_{i, \sigma}^{n}$ instead of $u_{\sigma}^{n}$ in order to avoid all confusion. Notice that $u_{i, \sigma}^{n}$ may be non zero for $\sigma \in \mathscr{E}_{\text {int }}^{(i)}$ while it is zero whenever $\sigma \in \mathscr{E}_{\text {ext }}^{(i)}$. In the same way, given $\varrho \in Y_{\mathscr{M}, \delta t}$ associated to the discrete density unknowns $\left\{\varrho_{K}^{n}, K \in \mathscr{M}, n \in\{1, \cdots, N\}\right\}$ we denote by $\varrho^{n} \in L_{\mathscr{M}}$ the piecewise constant function defined by $\varrho^{n}(\boldsymbol{x})=\varrho_{K}^{n}$ for $\boldsymbol{x} \in K, K \in \mathscr{M}$.

Finally, the discrete initial condition $\left(\varrho^{0}, \boldsymbol{u}^{0}\right) \in L_{\mathscr{M}} \times \mathbf{H}_{\mathscr{C}, 0}$ is such that $\varrho^{0}>0$ and the discrete initial total mass and energy are respectively defined by

$$
M_{0, \mathscr{M}}=\int_{\Omega} \varrho^{0} \mathrm{~d} \boldsymbol{x}, \quad \mathscr{E}_{0, \mathscr{M}}=\int_{\Omega} \frac{1}{2} \varrho^{0}\left|\boldsymbol{u}^{0}\right|^{2} \mathrm{~d} \boldsymbol{x}+\int_{\Omega} \mathscr{H}\left(\varrho^{0}\right) \mathrm{d} \boldsymbol{x}
$$

\subsection{The numerical scheme}

We consider an implicit-in-time scheme, which reads in its fully discrete form, for $1 \leq n \leq N$ and $1 \leq i \leq d$ :

$$
\begin{aligned}
& \frac{1}{\delta t}\left(\varrho^{n}-\varrho^{n-1}\right)+\operatorname{div}_{\mathscr{M}}^{\text {up }}\left(\varrho^{n} \boldsymbol{u}^{n}\right)=0, \\
& \frac{1}{\delta t}\left(\widehat{\varrho}^{(i)} u_{i}^{n}-\widehat{\varrho}^{n-1}(i) u_{i}^{n-1}\right)+\operatorname{div}_{\mathscr{E}}^{(i)}\left(\varrho^{n} \boldsymbol{u}^{n} u_{i}^{n}\right)-\mu \Delta_{\mathscr{E}}^{(i)} u_{i}^{n} \\
& -(\mu+\lambda) \check{\partial}_{i} \operatorname{div}_{\mathscr{M}} \boldsymbol{u}^{n}+\check{\partial}_{i} p\left(\varrho^{n}\right)=0,
\end{aligned}
$$

where the terms introduced for each discrete equation are defined hereafter.

\subsubsection{Mass balance equation}

Equation (3.10a) is a finite volume discretization of the mass balance $(1.1 a)$ over the primal mesh. The discrete "upwind" divergence is defined by

$$
\begin{array}{l|l}
\operatorname{div}_{\mathscr{M}}^{\text {up }}: & \quad L_{\mathscr{M}} \times \mathbf{H}_{\mathscr{E}, 0} \longrightarrow L_{\mathscr{M}} \\
& (\varrho, \boldsymbol{u}) \longmapsto \operatorname{div}_{\mathscr{M}}^{\mathrm{up}_{1}}(\varrho \boldsymbol{u})=\sum_{K \in \mathscr{M}} \frac{1}{|K|} \sum_{\sigma \in \mathscr{E}(K)} F_{\sigma, K}(\varrho, \boldsymbol{u}) \mathscr{X}_{K},
\end{array}
$$

where $F_{\sigma, K}(\varrho, \boldsymbol{u})$ stands for the mass flux across $\sigma$ outward $K$, which, because of the Dirichlet boundary conditions, vanishes on external faces and is given on the internal faces by:

$$
\forall \sigma=K\left|L \in \mathscr{E}_{\text {int }}, \quad F_{\sigma, K}(\varrho, \boldsymbol{u})=\right| \sigma \mid \varrho_{\sigma}^{\text {up }} u_{\sigma, K}
$$

where $u_{\sigma, K}$ is an approximation of the normal velocity to the face $\sigma$ outward $K$, defined by:

$$
u_{\sigma, K}=u_{\sigma} \boldsymbol{e}^{(i)} \cdot \boldsymbol{n}_{\sigma, K} \text { for } \sigma \in \mathscr{E}^{(i)} \cap \mathscr{E}(K) .
$$

Thanks to the boundary conditions, $u_{\sigma, K}$ vanishes for any external face $\sigma$. The density at the internal face $\sigma=K \mid L$ is obtained by an upwind technique:

$$
\varrho_{\sigma}^{\text {up }}=\mid \begin{array}{ll}
\varrho_{K} & \text { if } u_{\sigma, K} \geq 0 \\
\varrho_{L} & \text { otherwise. }
\end{array}
$$

Note that any solution $\left(\varrho^{n}, \boldsymbol{u}^{n}\right) \in L_{\mathscr{M}} \times \mathbf{H}_{\mathscr{C}, 0}$ to $(3.10 a)$ satisfy $\varrho_{K}^{n}>0, \forall K \in \mathscr{M}$ provided $\varrho_{K}^{n-1}>$ $0, \forall K \in \mathscr{M}$ and in particular $p\left(\varrho^{n}\right)$ makes sense. The positivity of the density $\varrho^{n}$ in $(3.10 a)$ is not enforced in the scheme but results from the above upwind choice. Indeed, for a given velocity field, the discrete mass balance $(3.10 a)$ is a linear system for $\varrho^{n}$ the matrix of which is an invertible matrix with a non negative inverse [20, Lemma C.3].

Note also that, with this definition, we have the usual finite volume property of local conservativity of the flux through a primal face $\sigma=K \mid L$ i.e.

$$
F_{\sigma, K}(\varrho, \boldsymbol{u})=-F_{\sigma, L}(\varrho, \boldsymbol{u})
$$


Consequently, summing (3.10a) over $K \in \mathscr{M}$ immediately yields the total conservation of mass, which reads:

$$
\forall n=1, \ldots N, \quad \int_{\Omega} \varrho^{n} \mathrm{~d} \boldsymbol{x}=\int_{\Omega} \varrho^{0} \mathrm{~d} \boldsymbol{x} .
$$

This is a discrete version of (2.7).

\subsubsection{The momentum equation}

We now turn to the discrete momentum balances (3.10b), which are obtained by discretizing the momentum balance equation (1.1b) on the dual cells associated to the faces of the mesh.

The discrete convective operator - The discrete divergence of the convective term $\varrho u \otimes u$ is defined by

$$
\begin{array}{l|l}
\operatorname{div}_{\mathscr{E}}^{\text {up }}: & \begin{array}{l}
L_{\mathscr{M}} \times \mathbf{H}_{\mathscr{C}, 0} \longrightarrow \mathbf{H}_{\mathscr{C}, 0} \\
\end{array} \quad(\varrho, \boldsymbol{u}) \longmapsto \operatorname{div}_{\mathscr{E}}^{\text {up }}(\varrho \boldsymbol{u} \otimes \boldsymbol{u})=\left(\operatorname{div}_{\mathscr{E}}^{(1)}\left(\varrho \boldsymbol{u} u_{1}\right), \ldots, \operatorname{div}_{\mathscr{E}}^{(d)}\left(\varrho \boldsymbol{u} u_{d}\right)\right),
\end{array}
$$

where for any $1 \leq i \leq d$, the $i^{\text {th }}$ component of the above operator reads:

$$
\begin{array}{l|l}
\operatorname{div}_{\mathscr{E}}^{(i)}: & L_{\mathscr{M}} \times \mathbf{H}_{\mathscr{E}, 0} \longrightarrow H_{\mathscr{E}, 0}^{(i)} \\
& (\varrho, \boldsymbol{u}) \longmapsto \operatorname{div}_{\mathscr{E}}^{(i)}\left(\varrho \boldsymbol{u} u_{i}\right)=\sum_{\sigma \in \mathscr{E}_{\text {int }}^{(i)}} \frac{1}{\left|D_{\sigma}\right|} \sum_{\epsilon \in \tilde{\mathscr{E}}\left(D_{\sigma}\right)} F_{\epsilon, \sigma}(\varrho, \boldsymbol{u}) u_{\epsilon} \mathscr{X}_{D_{\sigma}},
\end{array}
$$

where the quantity $F_{\epsilon, \sigma}=F_{\epsilon, \sigma}(\varrho, \boldsymbol{u})$ stands for a mass flux through the dual faces of the mesh and $u_{\epsilon}$ stands for an approximation of $i^{t h}$ component of the velocity over $\epsilon$. These quantities are defined in (3.19), (3.20), (3.25).

Let $\sigma \in \mathscr{E}_{\text {int }}^{(i)}$ (without loss of generality). The dual flux $F_{\epsilon, \sigma}(\varrho, \boldsymbol{u})$ is defined as follows

- First case - The vector $\boldsymbol{e}^{(i)}$ is normal to $\epsilon$, so $\epsilon$ is included in a primal cell $K$, and we denote by $\sigma^{\prime}$ the second face of $K$ which, in addition to $\sigma$, is normal to $\boldsymbol{e}^{(i)}$. We thus have $\epsilon=D_{\sigma} \mid D_{\sigma^{\prime}}$ (see Figure 3.3.2). Then the mass flux through $\epsilon$ is given by:

$$
F_{\epsilon, \sigma}(\varrho, \boldsymbol{u})=\frac{1}{2}\left[F_{\sigma, K}(\varrho, \boldsymbol{u}) \boldsymbol{n}_{\epsilon, D_{\sigma}} \cdot \boldsymbol{n}_{\sigma, K}+F_{\sigma^{\prime}, K}(\varrho, \boldsymbol{u}) \boldsymbol{n}_{\epsilon, D_{\sigma}} \cdot \boldsymbol{n}_{\sigma^{\prime}, K}\right] .
$$

where $\boldsymbol{n}_{\epsilon, D_{\sigma}}$ stands for the unit normal vector to $\epsilon$ outward $D_{\sigma}$.

- Second case - The vector $\boldsymbol{e}^{(i)}$ is tangent to $\epsilon$, and $\epsilon$ is the union of the halves of two primal faces $\tau$ and $\tau^{\prime}$ such that $\tau \in \mathscr{E}(K)$ and $\tau^{\prime} \in \mathscr{E}(L)$ (see Figure 3.3.2). The mass flux through $\epsilon$ is then given by:

$$
F_{\epsilon, \sigma}(\varrho, \boldsymbol{u})=\frac{1}{2}\left[F_{\tau, K}(\varrho, \boldsymbol{u})+F_{\tau^{\prime}, L}(\varrho, \boldsymbol{u})\right]
$$

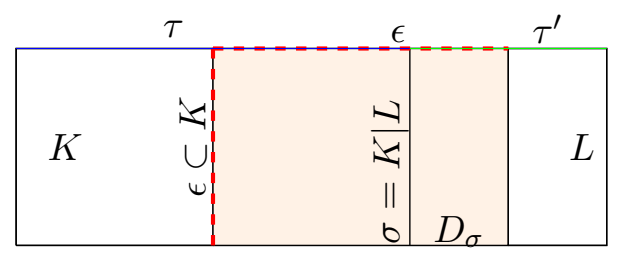

Figure 3: Notations for the dual fluxes of the first component of the velocity.

We notice that the sum over $\epsilon \in \tilde{\mathscr{E}}\left(D_{\sigma}\right)$ in formula (3.18) can be replaced by the sum over $\epsilon \in \tilde{\mathscr{E}}_{\text {int }}\left(D_{\sigma}\right)$.

Note that, with this definition, we have the usual finite volume property of local conservativity of the flux through a dual face $\epsilon=D_{\sigma} \mid D_{\sigma^{\prime}}$ i.e.

$$
F_{\epsilon, \sigma}(\varrho, \boldsymbol{u})=-F_{\epsilon, \sigma^{\prime}}(\varrho, \boldsymbol{u}),
$$

and vanish through a dual face included in the boundary of $\Omega$. 
In what follows we shall often use the abbreviated notation

$$
F_{\sigma, K}\left(\varrho^{n}, \mathbf{u}^{n}\right)=F_{\sigma, K}^{n}, \quad F_{\epsilon, \sigma}\left(\varrho^{n}, \mathbf{u}^{n}\right)=F_{\epsilon, \sigma}^{n} .
$$

The density on a dual cell is given by:

$$
\begin{array}{ll}
\text { for } \sigma \in \mathscr{E}_{\text {int }}, \sigma=K \mid L & \left|D_{\sigma}\right| \varrho_{D_{\sigma}}=\left|D_{\sigma, K}\right| \varrho_{K}+\left|D_{\sigma, L}\right| \varrho_{L}, \\
\text { for } \sigma \in \mathscr{E}_{\text {ext }}, \sigma \in \mathscr{E}(K), \quad & \varrho_{D_{\sigma}}=\varrho_{K} .
\end{array}
$$

and we denote

$$
\text { for } 1 \leq i \leq d, \quad \widehat{\varrho}^{(i)}=\sum_{\sigma \in \mathscr{\mathscr { C }}(i)} \varrho_{D_{\sigma}} \mathscr{X}_{D_{\sigma}}
$$

These definitions of the dual mass fluxes and the dual densities ensures that a finite volume discretization of the mass balance equation over the diamond cells holds:

$$
\forall 1 \leq i \leq d, \forall \sigma \in \mathscr{E}_{\text {int }}^{(i)}, \quad \frac{1}{\delta t}\left(\varrho_{D_{\sigma}}^{n}-\varrho_{D_{\sigma}}^{n-1}\right)+\frac{1}{\left|D_{\sigma}\right|} \sum_{\epsilon \in \widetilde{\mathscr{E}}\left(D_{\sigma}\right)} F_{\epsilon, \sigma}^{n}=0 .
$$

This is a necessary condition to be able to derive a discrete kinetic energy balance (see Theorem 4.1).

Since the flux across a dual face lying on the boundary is zero, the values $u_{\epsilon}$ are only needed at the internal dual faces, and we make the centered choice for their discretization, i.e., for $\epsilon=D_{\sigma} \mid D_{\sigma^{\prime}} \in \tilde{\mathscr{E}}_{\text {int }}^{(i)}$,

$$
u_{\epsilon} \equiv u_{i, \epsilon}=\frac{u_{\sigma}+u_{\sigma^{\prime}}}{2} \equiv \frac{u_{i, \sigma}+u_{i, \sigma^{\prime}}}{2} .
$$

Discrete divergence and gradient - The discrete divergence operator $\operatorname{div} \mathscr{M}$ is defined by:

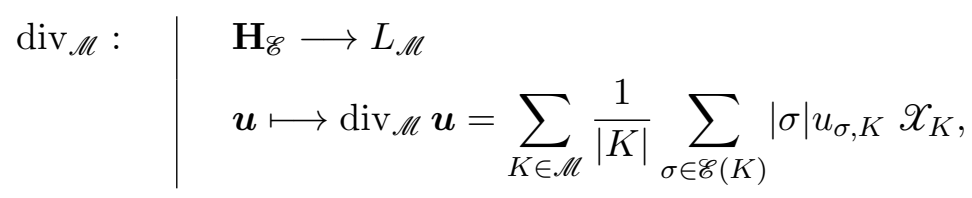

where $u_{\sigma, K}$ is defined in (3.13).

The discrete divergence of $\boldsymbol{u}=\left(u_{1}, \ldots, u_{d}\right) \in \mathbf{H}_{\mathscr{E}, 0}$ may also be written as

$$
\operatorname{div}_{\mathscr{M}} \boldsymbol{u}=\sum_{i=1}^{d} \sum_{K \in \mathscr{M}}\left(\Im_{i} u_{i}\right)_{K} \mathscr{X}_{K},
$$

where the discrete derivative $\left(\varpi_{i} u_{i}\right)_{K}$ of $u_{i}$ on $K$ is defined by

$$
\left(\varlimsup_{i} u_{i}\right)_{K}=\frac{|\sigma|}{|K|}\left(u_{\sigma^{\prime}}-u_{\sigma}\right) \text { with } K=\left[\overrightarrow{\sigma \sigma^{\prime}}\right], \sigma, \sigma^{\prime} \in \mathscr{E}^{(i)} \text {. }
$$

The gradient in the discrete momentum balance equation is defined as follows:

$$
\begin{array}{l|l}
\nabla_{\mathscr{E}}: & L_{\mathscr{M}} \longrightarrow \mathbf{H}_{\mathscr{E}, 0} \\
& p \longmapsto \nabla_{\mathscr{E}} p \\
& \nabla_{\mathscr{E}} p(\boldsymbol{x})=\left(\check{\partial}_{1} p(\boldsymbol{x}), \ldots, \partial_{d} p(\boldsymbol{x})\right)^{t},
\end{array}
$$

where $ð_{i} p \in H_{\mathscr{C}, 0}^{(i)}$ is the discrete derivative of $p$ in the $i$-th direction, defined by:

$$
\varlimsup_{i} p(\boldsymbol{x})=\frac{|\sigma|}{\left|D_{\sigma}\right|}\left(p_{L}-p_{K}\right) \quad \forall \boldsymbol{x} \in D_{\sigma}, \text { for } \sigma=\overrightarrow{K \mid L} \in \mathscr{E}_{\text {int }}^{(i)}, i=1, \ldots, d .
$$

Note that in fact, the discrete gradient of a function of $L_{\mathscr{M}}$ must be defined on the internal faces, and does not need to be defined on the external faces; we set it here in $\mathbf{H}_{\mathscr{E}, 0}$ (that is zero on the external faces) for the sake of simplicity.

The gradient in the discrete momentum balance equation is built as the dual operator of the discrete divergence which means (see [23]): 
Lemma 3.1 (Discrete div $-\nabla$ duality). Let $q \in L_{\mathscr{M}}$ and $\boldsymbol{v} \in \mathbf{H}_{\mathscr{C}, 0}$ then we have:

$$
\int_{\Omega} q \operatorname{div} \mathscr{M} \boldsymbol{v} \mathrm{d} \boldsymbol{x}+\int_{\Omega} \nabla_{\mathscr{E}} q \cdot \boldsymbol{v} \mathrm{d} \boldsymbol{x}=0 .
$$

Discrete Laplace operator - For $i=1 \ldots, d$, we classically define the discrete Laplace operator of the discrete $i$-th velocity component by:

$$
\begin{aligned}
& \begin{array}{l|l}
-\Delta_{\mathscr{E}}^{(i)}: & H_{\mathscr{E}, 0}^{(i)} \longrightarrow H_{\mathscr{E}, 0}^{(i)} \\
& u_{i} \longmapsto-\Delta_{\mathscr{E}}^{(i)} u_{i}
\end{array} \\
& -\Delta_{\mathscr{E}}^{(i)} u_{i}(\boldsymbol{x})=\frac{1}{\left|D_{\sigma}\right|} \sum_{\epsilon \in \tilde{\mathscr{E}}\left(D_{\sigma}\right)} \phi_{\epsilon, \sigma}\left(u_{i}\right), \quad \forall \boldsymbol{x} \in D_{\sigma}, \quad \text { for } \sigma \in \mathscr{E}_{\mathrm{int}}^{(i)}
\end{aligned}
$$

where $\tilde{\mathscr{E}}\left(D_{\sigma}\right)$ denotes the set of faces of $D_{\sigma}$, and

$$
\phi_{\epsilon, \sigma}\left(u_{i}\right)= \begin{cases}\frac{|\epsilon|}{d_{\epsilon}}\left(u_{\sigma}-u_{\sigma^{\prime}}\right) & \text { if } \epsilon=\sigma \mid \sigma^{\prime} \in \widetilde{\mathscr{E}}_{\text {int }}^{(i)}, \\ \frac{|\epsilon|}{d_{\epsilon}} u_{\sigma} & \text { if } \epsilon \in \widetilde{\mathscr{E}}_{\mathrm{ext}}^{(i)} \cap \tilde{\mathscr{E}}\left(D_{\sigma}\right)\end{cases}
$$

where $d_{\epsilon}$ is defined by (3.5). Note that we have the usual finite volume property of local conservativity of the flux through an interface $\epsilon=\sigma \mid \sigma^{\prime}$ :

$$
\phi_{\epsilon, \sigma}\left(u_{i}\right)=-\phi_{\epsilon, \sigma^{\prime}}\left(u_{i}\right), \quad \forall \epsilon=\sigma \mid \sigma^{\prime} \in \tilde{\mathscr{E}}_{\text {int }}^{(i)} .
$$

Then the discrete Laplace operator of the full velocity vector is defined by

$$
\begin{aligned}
-\Delta_{\mathscr{C}}: & \mathbf{H}_{\mathscr{C}, 0} \longrightarrow \mathbf{H}_{\mathscr{E}, 0} \\
& \boldsymbol{u} \mapsto-\Delta_{\mathscr{C}} \boldsymbol{u}=\left(-\Delta_{\mathscr{E}}^{(1)} u_{1}, \ldots,-\Delta_{\mathscr{E}}^{(d)} u_{d}\right)^{t} .
\end{aligned}
$$

Let us now recall the definition of the discrete $H_{0}^{1}$ inner product [11]; it is obtained by multiplying the discrete Laplace operator scalarly by a test function $\boldsymbol{v} \in \mathbf{H}_{\mathscr{C}, 0}$ and integrating over the computational domain. A simple reordering of the sums (which may be seen as a discrete integration by parts) yields, thanks to the conservativity of the diffusion flux (3.34):

$$
\begin{aligned}
& \forall(\boldsymbol{u}, \boldsymbol{v}) \in \mathbf{H}_{\mathscr{E}, 0} 0^{2}, \quad \int_{\Omega}-\Delta_{\mathscr{E}} \boldsymbol{u} \cdot \boldsymbol{v} \mathrm{d} \boldsymbol{x}=[\boldsymbol{u}, \boldsymbol{v}]_{1, \mathscr{E}, 0}=\sum_{i=1}^{d}\left[u_{i}, v_{i}\right]_{1, \mathscr{E}(i), 0}, \\
& \text { with }\left[u_{i}, v_{i}\right]_{1, \mathscr{E}(i), 0}=\sum_{\substack{\epsilon \in \widetilde{\mathscr{C}}_{\text {int }}^{(i)} \\
\epsilon=\sigma \sigma^{\prime}}} \frac{|\epsilon|}{d_{\epsilon}}\left(u_{\sigma}-u_{\sigma^{\prime}}\right)\left(v_{\sigma}-v_{\sigma^{\prime}}\right)+\sum_{\substack{\epsilon \in \widetilde{\mathscr{C}}_{\text {ext }}(i) \\
\epsilon \in \tilde{\mathscr{E}}\left(D_{\sigma}\right)}} \frac{|\epsilon|}{d_{\epsilon}} u_{\sigma} v_{\sigma}
\end{aligned}
$$

The bilinear forms $\mid \begin{aligned} & H_{\mathscr{E}, 0}^{(i)} \times H_{\mathscr{E}, 0}^{(i)} \rightarrow \mathbb{R} \\ & (u, v) \mapsto\left[u_{i}, v_{i}\right]_{1, \mathscr{E}(i), 0}\end{aligned}$ and $\mid \begin{aligned} & \mathbf{H}_{\mathscr{E}, 0} \times \mathbf{H}_{\mathscr{E}, 0} \rightarrow \mathbb{R} \\ & (\boldsymbol{u}, \boldsymbol{v}) \mapsto[\boldsymbol{u}, \boldsymbol{v}]_{1, \mathscr{E}, 0}\end{aligned} \quad$ are inner products on $H_{\mathscr{\mathscr { C }}, 0}^{(i)}$ and $\mathbf{H}_{\mathscr{C}, 0}$ respectively, which induce the following discrete $H_{0}^{1}$ norms:

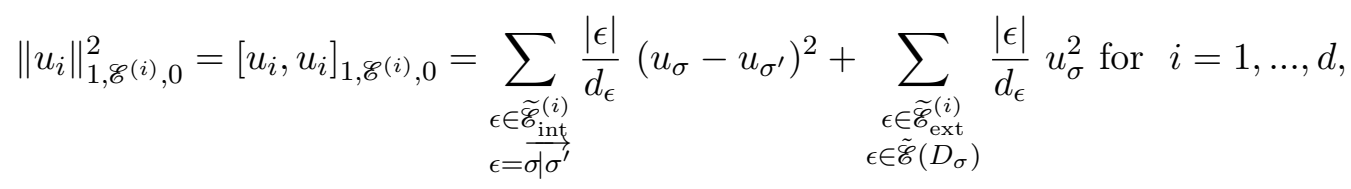

$$
\begin{aligned}
& \|\boldsymbol{u}\|_{1, \mathscr{E}, 0}^{2}=[\boldsymbol{u}, \boldsymbol{u}]_{1, \mathscr{C}, 0}=\sum_{i=1}^{d}\left\|u_{i}\right\|_{1, \mathscr{E}(i), 0}^{2} .
\end{aligned}
$$

Since we are working on Cartesian grids, this inner product may be formulated as the $L^{2}$ inner product 

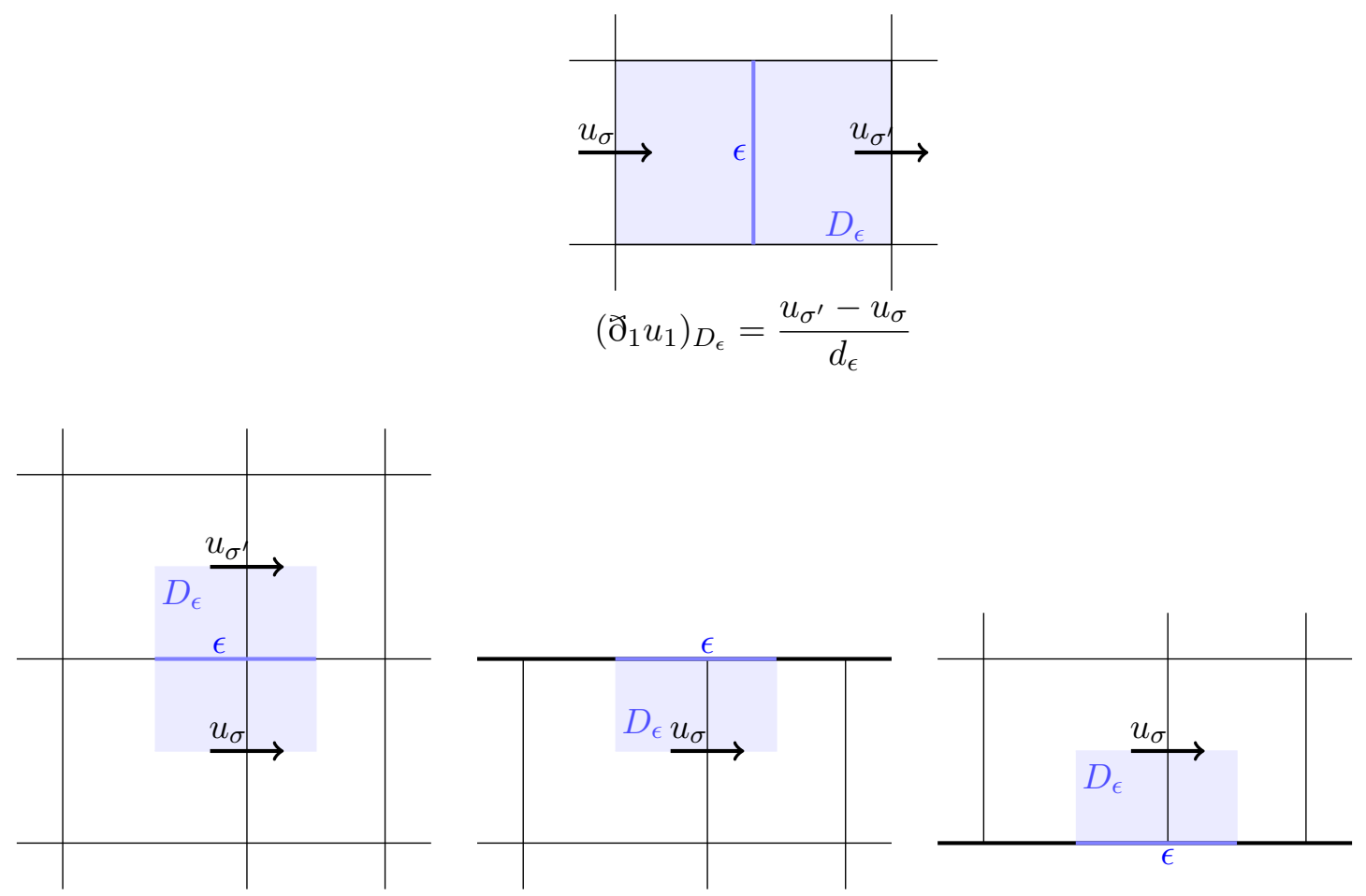

$\left(\check{\partial}_{2} u_{1}\right)_{D_{\epsilon}}=\frac{u_{\sigma^{\prime}}-u_{\sigma}}{d_{\epsilon}}$

$\left(\check{\partial}_{2} u_{1}\right)_{D_{\epsilon}}=\frac{-u_{\sigma}}{d_{\epsilon}}$

$\left(ð_{2} u_{1}\right)_{D_{\epsilon}}=\frac{u_{\sigma}}{d_{\epsilon}}$

Figure 4: Notations for the definition of the partial space derivatives of the first component of the velocity, in two space dimensions.

of discrete gradients. Indeed, consider the following discrete gradient of each velocity component $u_{i}$.

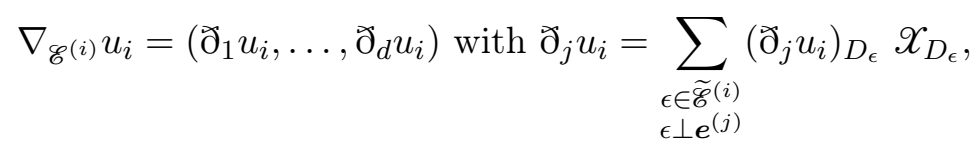

where the elements $D_{\epsilon}$ of the bi-dual grid are defined in (3.4) (see also Figure 2) and

$$
\left(\varpi_{j} u_{i}\right)_{D_{\epsilon}}=\left\{\begin{array}{l}
\frac{u_{\sigma^{\prime}}-u_{\sigma}}{d_{\epsilon}} \text { with } \epsilon=\overrightarrow{\sigma \mid \sigma^{\prime}}, \\
-\frac{u_{\sigma}}{d_{\epsilon}} \boldsymbol{e}^{(j)} \cdot \boldsymbol{n}_{\epsilon, D_{\sigma}} \text { with } \epsilon \in \widetilde{\mathscr{E}}_{\mathrm{ext}}^{(i)} \cap \tilde{\mathscr{E}}\left(D_{\sigma}\right),
\end{array}\right.
$$

where $\boldsymbol{n}_{\epsilon, D_{\sigma}}$ stands for the unit normal vector to $\epsilon$ outward $D_{\sigma}$, see Figure 4 . This definition is compatible with the definition of the discrete derivative $\left(\Im_{i} u_{i}\right)_{K}$ given by (3.28), since, if $\epsilon \subset K$ then $D_{\epsilon}=K$. Note that the second line in (3.39) is zero provide $i=j$, or provided $\sigma \in \mathscr{E}_{\text {ext }}^{(i)}, \epsilon \perp \mathbf{e}^{(j)}$ with $i \neq j$. With this definition, it is easily seen that

$$
\int_{\Omega} \nabla_{\mathscr{E}(i)} u \cdot \nabla_{\mathscr{E}(i)} v \mathrm{~d} \boldsymbol{x}=[u, v]_{1, \mathscr{C}(i), 0}, \forall u, v \in H_{\mathscr{C}, 0}^{(i)}, \forall i=1, \ldots, d .
$$

where $[u, v]_{1, \mathscr{E}(i), 0}$ is the discrete $H_{0}^{1}$ inner product defined by (3.36). We may then define

$$
\nabla_{\mathscr{E}} \boldsymbol{u}=\left(\nabla_{\mathscr{E}(1)} u_{1}, \ldots, \nabla_{\mathscr{E}(d)} u_{d}\right),
$$

so that

$$
\int_{\Omega} \nabla_{\mathscr{C}} \boldsymbol{u}: \nabla_{\mathscr{C}} \boldsymbol{v} \mathrm{d} \boldsymbol{x}=[\boldsymbol{u}, \boldsymbol{v}]_{1, \mathscr{E}, 0} .
$$

We will need discrete Sobolev inequalites for the discrete approximations. The following lemma is proved in [11]. 
Lemma 3.2 (Discrete Sobolev inequalities). Let $\Omega$ be a bounded domain of $\mathbb{R}^{d}, d=2$ or $d=3$, adapted to the $M A C$-scheme (that is any finite union of rectangles in $2 D$ or rectangular in $3 D$ ), and let $\mathscr{D}=(\mathscr{M}, \mathscr{E})$ be a MAC grid of $\Omega$. Let $q<+\infty$ if $d=2$ and $q=6$ if $d=3$. Then there exists $c=c\left(q,|\Omega|, \eta_{\mathscr{M}}\right)$ depending on $\eta_{\mathscr{M}}$ in a nondecreasing way such that, for all $u \in \mathbf{H}_{\mathscr{E}, 0}$,

$$
\|\boldsymbol{u}\|_{L^{q}(\Omega)} \leq c\|\boldsymbol{u}\|_{1, \mathscr{\ell}, 0} .
$$

\subsection{Projection operators}

In this section we introduce several projection operators. We first define the mean-value interpolator over $L_{\mathscr{M}}$ :

$$
\mathscr{P}_{\mathscr{M}}: \quad \begin{array}{lll}
L_{\mathrm{loc}}^{1}(\Omega) & \longrightarrow & L_{\mathscr{M}} \\
\varphi & \mapsto & \varphi_{\mathscr{M}}:=\mathscr{P}_{\mathscr{M}} \varphi=\sum_{K \in \mathscr{M}} \varphi_{K} \mathscr{X}_{K},
\end{array}
$$

with

$$
\varphi_{K}=\frac{1}{|K|} \int_{K} \varphi(\boldsymbol{x}) \mathbf{d} \boldsymbol{x}, \forall K \in \mathscr{M} .
$$

This operator satisfies for any $1 \leq p \leq \infty$ and for any $r \in L^{p}(\Omega)$,

$$
\left\|\mathscr{P}_{\mathscr{M}} r\right\|_{L^{p}(\Omega)} \leq\|r\|_{L^{p}(\Omega)} .
$$

We also define over $H_{\mathscr{E}, 0}^{(i)}$ the following interpolation operator $\mathscr{P}_{\mathscr{E}}^{(i)}$ :

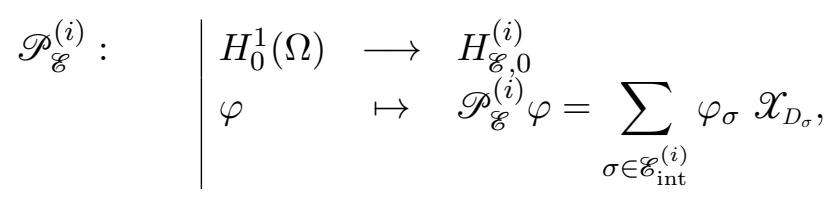

with

$$
\varphi_{\sigma}=\frac{1}{|\sigma|} \int_{\sigma} \varphi(\boldsymbol{x}) \mathrm{d} \gamma(\boldsymbol{x}), \forall \sigma \in \mathscr{E}_{\text {int }}^{(i)}
$$

where $\mathrm{d} \gamma(\boldsymbol{x})$ is the $d$-1-Lebesgue measure on $\sigma$ and we denote

$$
\mathscr{P}_{\mathscr{E}}=\left(\mathscr{P}_{\mathscr{E}}^{(1)}, \ldots, \mathscr{P}_{\mathscr{E}}^{(d)}\right) \in \mathscr{L}\left(H_{0}^{1}(\Omega)^{d}, \mathbf{H}_{\mathscr{E}, 0}\right), \boldsymbol{v}_{\mathscr{E}}:=\mathscr{P}_{\mathscr{E}} \boldsymbol{v}
$$

its vector valued extension. This operator preserves the divergence in the following sense (see [22]):

$$
\forall \boldsymbol{v} \in H_{0}^{1}(\Omega)^{d}, \forall q \in L_{\mathscr{M}}, \int_{\Omega} q \operatorname{div}_{\mathscr{M}} \mathscr{P}_{\mathscr{C}} \boldsymbol{v} \mathrm{d} \boldsymbol{x}=\int_{\Omega} q \operatorname{div} \boldsymbol{v} \mathrm{d} \boldsymbol{x} .
$$

This operator satisfies for any $1 \leq p \leq \infty$ and for any $\boldsymbol{U} \in W_{0}^{1, p}(\Omega)^{d}$,

$$
\left\|\mathscr{P}_{\mathscr{C}} \boldsymbol{U}\right\|_{L^{p}(\Omega)^{d}} \leq c\|\nabla \boldsymbol{U}\|_{L^{p}(\Omega)^{d \times d}} .
$$

where the constant $c$ depends on $d$ and $p$ and on $|\Omega|$.

In the following Lemma we recall some classical mean value inequalities. These inequalities are used to obtain estimates involving the projector operators $\mathscr{P}_{\mathscr{M}}$ and $\mathscr{P}_{\mathscr{E}}$ previously defined.

Lemma 3.3. [Mean value inequalities]

1. Let $D=\prod_{i=1}^{d}\left(a_{i}, b_{i}\right)$ be a bounded open square of $\mathbb{R}^{d}, d \geq 1$. Let $\sigma \subset \partial D$ be a face of $D$. Let $1 \leq p \leq \infty$. There exists $c$ only depending on $d$ and $p$ such that $\forall v \in W^{1, p}(D)$,

$$
\begin{aligned}
& \left\|v-v_{\sigma}\right\|_{L^{p}(D)} \leq c \operatorname{diam}(D)\|\nabla v\|_{L^{p}(D)^{d}} \\
& \left\|v-v_{D}\right\|_{L^{p}(D)} \leq c \operatorname{diam}(D)\|\nabla v\|_{L^{p}(D)^{d}}
\end{aligned}
$$


where $v_{D}=\frac{1}{|D|} \int_{D} v \mathrm{~d} \boldsymbol{x}$ and $v_{\sigma}=\frac{1}{|\sigma|} \int_{\sigma} v \mathrm{~d} \gamma(\boldsymbol{x})(\mathrm{d} \gamma(\boldsymbol{x})$ is the $d-1$-Lebesgue measure on $\sigma)$. Moreover if $v \in C^{2}(\bar{D})$ then

$$
\left|v_{D}-v\left(\boldsymbol{x}_{D}\right)\right| \leq\left\|\nabla^{2} v\right\|_{L^{\infty}(D) d \times d} \operatorname{diam}(D)^{2},
$$

where $\boldsymbol{x}_{D}$ stands for the center of mass of $D$ and

$$
\left|v\left(\frac{\boldsymbol{x}+\boldsymbol{y}}{2}\right)-\frac{1}{2}(v(\boldsymbol{x})+v(\boldsymbol{y}))\right| \leq \frac{1}{8}\left\|\nabla^{2} v\right\|_{L^{\infty}(\Omega) d \times d}|\boldsymbol{x}-\boldsymbol{y}|^{2}, \forall \boldsymbol{x}, \boldsymbol{y} \in \bar{D} .
$$

2. Let $\mathscr{D}=(\mathscr{M}, \mathscr{E})$ be a $M A C$ grid of the computational domain $\Omega$, let $1 \leq p \leq \infty$. Then there exsits $c$ only depending on $p$ and on $d$ such that for any $(r, \boldsymbol{U}) \in W^{1, p}(\Omega) \times W_{0}^{1, p}(\Omega)^{d}$,

$$
\begin{aligned}
\left\|r-\mathscr{P}_{\mathscr{M}} r\right\|_{L^{p}(\Omega)} & \leq c h_{\mathscr{M}}\|\nabla v\|_{L^{p}(\Omega)^{d}}, \\
\left\|\boldsymbol{U}-\mathscr{P}_{\mathscr{E}} \boldsymbol{U}\right\|_{L^{p}(\Omega)^{d}} & \leq c h_{\mathscr{M}}\|\nabla \boldsymbol{U}\|_{L^{p}(\Omega)^{d \times d}},
\end{aligned}
$$

3. There exists $c$ only depending on $d$ and $p$ and $|\Omega|$ and on $\eta_{\mathscr{M}}$ in a nondecreasing way such that for any $\boldsymbol{U} \in W_{0}^{1, p}(\Omega)^{d}$,

$$
\left\|\nabla_{\mathscr{C}} \mathscr{P}_{\mathscr{E}} \boldsymbol{U}\right\|_{L^{p}(\Omega) d \times d} \leq c\|\nabla \boldsymbol{U}\|_{L^{p}(\Omega) d \times d}
$$

Moreover if $\boldsymbol{U} \in C^{2}(\bar{\Omega})^{d}$,

$$
\left\|\oiint_{j} \mathscr{P}_{\mathscr{E}} U_{i}-\partial_{j} U_{i}\right\|_{L^{\infty}(\Omega)} \leq c h_{\mathscr{M}}\left\|\nabla^{2} \boldsymbol{U}_{i}\right\|_{L^{\infty}(\Omega)^{d \times d}} .
$$

Proof. Let us prove (3.50). Let us define the reference square $\hat{D}=(0,1)^{d}$ and let $\boldsymbol{\varphi}=\left(\varphi_{i}\right)_{1 \leq i \leq d}$ defined by

$$
\varphi_{i}\left(x_{i}\right)=\left(1-x_{i}\right) a_{i}+x_{i} b_{i}
$$

One has for any $v \in W^{1, p}(D)$

$$
\|v\|_{L^{p}(D)}=|D|^{\frac{1}{p}}\|v \circ \varphi\|_{L^{p}(\hat{D})} \quad\left\|\partial_{i} v\right\|_{L^{p}(D)}=\frac{|D|^{\frac{1}{p}}}{b_{i}-a_{i}}\left\|\partial_{i}(v \circ \varphi)\right\|_{L^{p}(\hat{D})} .
$$

Since $W^{1, p}(\hat{D})$ is compactly embedded in $L^{p}(\hat{D})$ there exists $c$ only depending on $d$ and $p$ such that for any $v \in W^{1, p}(\hat{D})$

$$
\left\|v-v_{\hat{D}}\right\|_{L^{p}(\hat{D})} \leq c\|\nabla v\|_{L^{p}(\hat{D})} .
$$

If $p=+\infty$ one has

$$
\left\|v-v_{D}\right\|_{L^{\infty}(D)}=\left\|v-v \circ \boldsymbol{\varphi}_{\hat{D}}\right\|_{L^{\infty}(D)}=\left\|v \circ \boldsymbol{\varphi}-v \circ \boldsymbol{\varphi}_{\hat{D}}\right\|_{L^{\infty}(\hat{D})} \leq c\|\nabla(v \circ \boldsymbol{\varphi})\|_{L^{\infty}(\hat{D})} .
$$

Using (3.57) we infer that

$$
\|\nabla(v \circ \varphi)\|_{L^{\infty}(\hat{D})} \leq c \operatorname{diam}(D)\|(\nabla v) \circ \varphi\|_{L^{\infty}(\hat{D})} \leq c \operatorname{diam}(D)\|\nabla v\|_{L^{\infty}(D)}
$$

which gives (3.50). If $1 \leq p<\infty$ one has for any $v \in W^{1, p}(D)$

$$
\left\|v-v_{D}\right\|_{L^{p}(D)}^{p}=\int_{D}\left|v-v_{D}\right|^{p} \mathrm{~d} \boldsymbol{x}=\int_{D}\left|v-v \circ \boldsymbol{\varphi}_{\hat{D}}\right|^{p} \mathrm{~d} \boldsymbol{x}=|D| \int_{\hat{D}}\left|v \circ \boldsymbol{\varphi}-v \circ \boldsymbol{\varphi}_{\hat{D}}\right|^{p} \mathrm{~d} \boldsymbol{x} \leq c|D|\|\nabla(v \circ \boldsymbol{\varphi})\|_{L^{p}(\hat{D})}^{p} .
$$

Using (3.57) we infer that

$$
\|\nabla(v \circ \varphi)\|_{L^{p}(\hat{D})}^{p} \leq c \operatorname{diam}(D)^{p}\|(\nabla v) \circ \varphi\|_{L^{p}(\hat{D})}^{p} \leq c \operatorname{diam}(D)^{p} \frac{1}{|D|}\|\nabla v\|_{L^{p}(D)}^{p}
$$

which also gives (3.50). The proof of (3.49) is similar. Let us prove (3.51). We have

$$
v_{D}-v\left(\boldsymbol{x}_{D}\right)=\frac{1}{2|D|} \int_{D}\left(\nabla^{2} v(c(\boldsymbol{x}))\left(\boldsymbol{x}-\boldsymbol{x}_{D}\right)\right) \cdot\left(\boldsymbol{x}-\boldsymbol{x}_{D}\right) \mathrm{d} \boldsymbol{x} .
$$


where $c(\boldsymbol{x}) \in \bar{D}$ wich gives

$$
\left|v_{D}-v\left(\boldsymbol{x}_{D}\right)\right| \leq\left\|\nabla^{2} v\right\|_{L^{\infty}(D)} d \times d \frac{1}{|D|} \int_{D}\left|\boldsymbol{x}-\boldsymbol{x}_{D}\right|^{2} \mathrm{~d} \boldsymbol{x} .
$$

Consequently

$$
\left|v_{D}-v\left(\boldsymbol{x}_{D}\right)\right| \leq\left\|\nabla^{2} v\right\|_{L^{\infty}(D) d \times d} \operatorname{diam}(D)^{2} .
$$

Let us prove (3.52). A Taylor expansion of the function $t \rightarrow \boldsymbol{v}(t \boldsymbol{x}+(1-t) \boldsymbol{y})$ gives

$$
\begin{aligned}
& v(\boldsymbol{x})=v\left(\frac{\boldsymbol{x}+\boldsymbol{y}}{2}\right)+\nabla v\left(\frac{\boldsymbol{x}+\boldsymbol{y}}{2}\right) \cdot \frac{\boldsymbol{x}-\boldsymbol{y}}{2}+\frac{1}{8}\left(\nabla^{2} v(\xi)(\boldsymbol{x}-\boldsymbol{y})\right) \cdot(\boldsymbol{x}-\boldsymbol{y}) . \\
& v(\boldsymbol{y})=v\left(\frac{\boldsymbol{x}+\boldsymbol{y}}{2}\right)+\nabla v\left(\frac{\boldsymbol{x}+\boldsymbol{y}}{2}\right) \cdot \frac{\boldsymbol{y}-\boldsymbol{x}}{2}+\frac{1}{8}\left(\nabla^{2} v(\tilde{\xi})(\boldsymbol{x}-\boldsymbol{y})\right) \cdot(\boldsymbol{x}-\boldsymbol{y}) .
\end{aligned}
$$

where $\xi, \tilde{\xi} \in[\boldsymbol{x}, \boldsymbol{y}]$. The expected result follows from the summation of the two previous identities. The proof of (3.53) and (3.54) are trivial consequences of (3.49) and (3.50). The proof of (3.55) can be found in [22]. Let us prove (3.56). By virtue of (3.4) one has

$$
\left\|\partial_{j} \mathscr{P}_{\mathscr{E}} U_{i}-\partial_{j} U_{i}\right\|_{L^{\infty}(\Omega)} \leq \max _{\epsilon \in \tilde{\mathscr{E}}^{(i, j)}}\left\|\partial_{j} \mathscr{P}_{\mathscr{E}} U_{i}-\partial_{j} U_{i}\right\|_{L^{\infty}\left(D_{\epsilon}\right)} .
$$

Moreover by virtue of (3.51) and (3.52) we can write for $\epsilon=\overrightarrow{\sigma \mid \sigma^{\prime}} \in \tilde{\mathscr{E}}_{\text {int }}^{(i)}, \epsilon \perp \boldsymbol{e}^{(j)}, \boldsymbol{x} \in D_{\epsilon}$,

$$
\begin{aligned}
\partial_{j}\left(\mathscr{P}_{\mathscr{E}}^{(i)} U_{i}\right)_{D_{\epsilon}}-\frac{\partial}{\partial x_{j}} U_{i}(\boldsymbol{x})= & \frac{1}{d_{\epsilon}}\left(\left(\mathscr{P}_{\mathscr{E}}^{(i)} U_{i}\right)_{\sigma^{\prime}}-\left(\mathscr{P}_{\mathscr{E}}^{(i)} U_{i}\right)_{\sigma}\right)-\frac{\partial}{\partial x_{j}} U_{i}(\boldsymbol{x}) \\
= & \frac{1}{d_{\epsilon}}\left(U_{i}\left(\boldsymbol{x}_{\sigma^{\prime}}\right)-U_{i}\left(\boldsymbol{x}_{\sigma}\right)\right)-\frac{\partial}{\partial x_{j}} U_{i}(\boldsymbol{x})+R_{\epsilon} \\
= & =\frac{\partial}{\partial x_{j}} U_{i}^{n}\left(\boldsymbol{x}_{\sigma, \sigma^{\prime}}\right)-\frac{\partial}{\partial x_{j}} U_{i}(\boldsymbol{x})+R_{\epsilon}^{n},
\end{aligned}
$$

where $\boldsymbol{x}_{\sigma, \sigma^{\prime}} \in\left[\boldsymbol{x}_{\sigma}, \boldsymbol{x}_{\sigma^{\prime}}\right]$, and where the remainder $R_{\epsilon}^{n}$ satisfies

$$
\left|R_{\epsilon}\right| \leq \operatorname{ch}_{\mathscr{M}}
$$

with $c>0$ dependent on $\eta_{\mathscr{M}}$ and on $\left\|\nabla^{2} \boldsymbol{U}\right\|_{L^{\infty}(\Omega) d \times d}$. Note that the case $\epsilon \in \tilde{\mathscr{E}}_{\text {ext }}^{(i)}$ can be treated in the same way. Consequently we have inequality

$$
\left\|\widetilde{\partial}_{j} \mathscr{P}_{\mathscr{E}} U_{i}-\partial_{j} U_{i}\right\|_{L^{\infty}\left(D_{\epsilon}\right)} \leq c h_{\mathscr{M}}, \forall(i, j) \in\{1,2,3\}^{2}, \forall \epsilon \in \tilde{\mathscr{E}}_{\text {int }}^{(i)}, \epsilon \perp \boldsymbol{e}^{(j)},
$$

where the constant $c$ depends on $\left\|\nabla^{2} \boldsymbol{U}\right\|_{L^{\infty}(\Omega)}$ and we obtain the expected result.

In the following defintion, we introduce two velocity interpolators.

Definition 3.3 (Velocity interpolators). 1. For a given $M A C$ grid $\mathscr{D}=(\mathscr{M}, \mathscr{E})$, we define, for $i, j=$ $1, \ldots, d$, the full grid velocity reconstruction operator with respect to $(i, j)$ by

$$
\begin{aligned}
\mathscr{R}_{\mathscr{G}}^{(i, j)}: & H_{\mathscr{E}, 0}^{(i)} \rightarrow H_{\mathscr{E}, 0}^{(j)} \\
& v \mapsto \mathscr{R}_{\mathscr{C}}^{(i, j)} v=\sum_{\sigma \in \mathscr{C}_{\text {int }}^{(j)}}\left(R_{\mathscr{C}}^{(i, j)} v\right)_{\sigma} \mathscr{X}_{D_{\sigma}},
\end{aligned}
$$

where

$$
\begin{aligned}
& \left(R_{\mathscr{E}}^{(i, j)} v\right)_{\sigma}=v_{\sigma} \text { if } \sigma \in \mathscr{E}_{\text {int }}^{(i)}, \quad\left(R_{\mathscr{E}}^{(i, j)} v\right)_{\sigma}=\frac{1}{\operatorname{card}\left(\mathcal{N}_{\sigma}\right)} \sum_{\sigma^{\prime} \in \mathcal{N}_{\sigma}} v_{\sigma^{\prime}} \text { otherwise, } \\
& \text { where, for any } \sigma \in \mathscr{E}_{\text {int }} \backslash \mathscr{E}_{\text {int }}^{(i)}, \mathscr{N}_{\sigma}=\left\{\sigma^{\prime} \in \mathscr{E}^{(i)}, D_{\sigma} \cap \sigma^{\prime} \neq \emptyset\right\} .
\end{aligned}
$$

We refer the reader to Figures 5 and 6 for the geometrical illustration of this notion. 
2. For any $i=1, \ldots d$, we also define a projector from $H_{\mathscr{E}}^{(i)}$ into $L_{\mathscr{M}}$ by

$$
\begin{aligned}
\mathscr{R}_{\mathscr{M}}^{(i)}: & H_{\mathscr{E}}^{(i)} \rightarrow L_{\mathscr{M}} \\
& v \mapsto \mathscr{R}_{\mathscr{M}}^{(i)} v=\sum_{K \in \mathscr{M}}\left(\mathscr{R}_{\mathscr{M}}^{(i)} v\right)_{K} \mathscr{X}_{K},
\end{aligned}
$$

where

$$
\left(\mathscr{R}_{\mathscr{M}}^{(i)} v\right)_{K}=\frac{1}{2} \sum_{\sigma \in \mathscr{\mathscr { C }}(i)} v_{\sigma} .
$$

We then define

$$
\begin{aligned}
& \mathscr{R}_{\mathscr{M}}: \mathbf{H}_{\mathscr{E}} \rightarrow L_{\mathscr{M}}^{d} \\
& \quad \boldsymbol{v}=\left(v_{1}, \ldots v_{d}\right) \mapsto \mathscr{R}_{\mathscr{M}} \boldsymbol{v}=\left(\mathscr{R}_{\mathscr{M}}^{(1)} v_{1}, \ldots, \mathscr{R}_{\mathscr{M}}^{(d)} v_{d}\right) .
\end{aligned}
$$

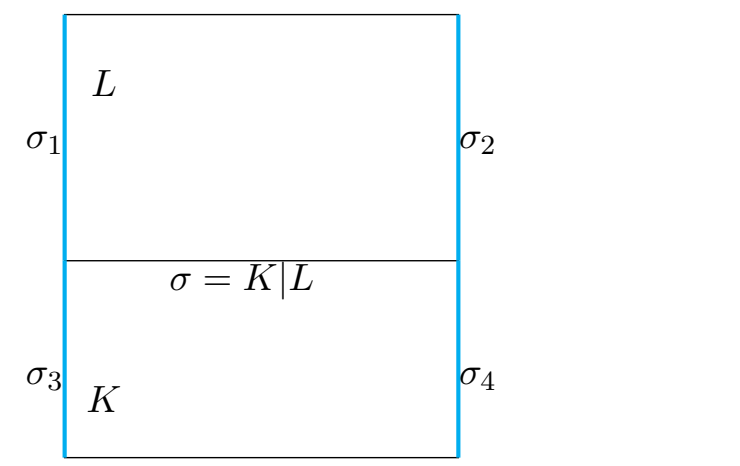

Figure 5: Set $\mathcal{N}_{\sigma}=\left\{\sigma_{1}, \sigma_{2}, \sigma_{3}, \sigma_{4}\right\}$ with $\sigma \in \mathscr{E}_{\text {int }}^{(j)}(K), j \neq i$ in two dimensions $(i=1, j=2)$

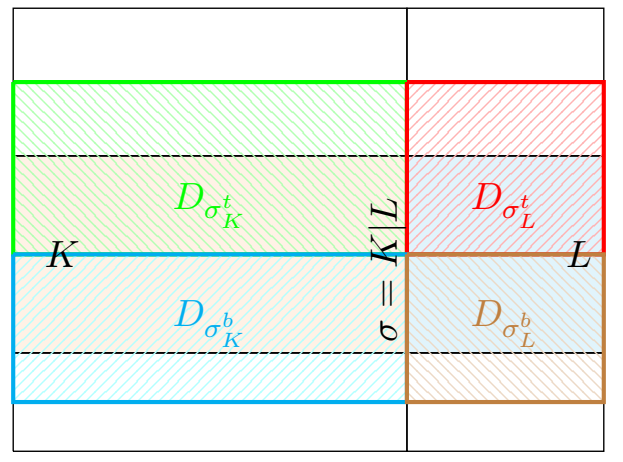

Figure 6: Full grid velocity interpolate.

These operators satisfy the following stability estimate (see [23]).

Lemma 3.4. There exists $c>0$, depending only on $d, p$ and on $\eta_{\mathscr{M}}$ in a nondecreasing way such that for any $i, j=1, \ldots, d$ and for any $v \in H_{\mathscr{E}, 0}^{(i)}$,

$$
\begin{gathered}
\left\|R_{\mathscr{C}}^{(i, j)} v\right\|_{L^{p}(\Omega)} \leq c\|v\|_{L^{p}(\Omega)}, 1 \leq p<\infty, \\
\left\|\mathscr{R}_{\mathscr{M}}^{(i)} v\right\|_{L^{p}(\Omega)} \leq \operatorname{ch}_{\mathscr{M}}\left\|\widetilde{\partial}_{i} v\right\|_{L^{p}(\Omega)}, 1 \leq p \leq \infty .
\end{gathered}
$$

Moreover, the following lemma holds:

Lemma 3.5. There exists $c>0$, depending only on $d, p$ and on $\eta_{\mathscr{M}}$ in a nondecreasing way such that for any $i, j=1, \ldots, d$ and for any $v \in H_{\mathscr{E}, 0}^{(i)}$,

$$
\left\|\mathscr{R}_{\mathscr{G}}^{(i, j)} v-v\right\|_{L^{p}(\Omega)} \leq c h_{\mathscr{M}}\left\|\nabla_{\mathscr{G}}(i) v\right\|_{L^{p}(\Omega)}, 1 \leq p<\infty,
$$

and

$$
\left\|\mathscr{R}_{\mathscr{M}}^{(i)} v-v\right\|_{L^{p}(\Omega)} \leq c h_{\mathscr{M}}\left\|ð_{i} v\right\|_{L^{p}(\Omega)}, 1 \leq p \leq \infty .
$$


Proof. Let us prove (3.67). From the definition of $\mathscr{R}_{\mathscr{M}}^{(i)}$ (see (3.62)) we have for $1 \leq p<\infty$

$$
\begin{array}{r}
\left\|\mathscr{R}_{\mathscr{M}}^{(i)} v-v\right\|_{L^{p}(\Omega)}^{p}=\sum_{K \in \mathscr{M}}\left\|\mathscr{R}_{\mathscr{M}}^{(i)} v-v\right\|_{L^{p}(K)}^{p}=\sum_{\substack{K=\left[\overrightarrow{\left.\sigma \sigma^{\prime}\right]} \\
\sigma, \sigma^{\prime} \in \mathscr{E}^{(i)}\right.}}\left(\left\|\mathscr{R}_{\mathscr{M}}^{(i)} v-v\right\|_{L^{p}\left(D_{\sigma, K}\right)}^{p}+\left\|\mathscr{R}_{\mathscr{M}}^{(i)} v-v\right\|_{L^{p}\left(D_{\sigma^{\prime}, K}\right)}^{p}\right) \\
\leq \frac{1}{2^{p}} h_{\mathscr{M}}^{p} \sum_{K \in \mathscr{M}}\left\|{ }_{\boldsymbol{\partial}_{i}} v\right\|_{L^{p}(K)}^{p} \leq c h_{\mathscr{M}}^{p}\left\|\partial_{i} v\right\|_{L^{p}(\Omega)}^{p}
\end{array}
$$

which gives the expected result. For $p=+\infty$ we have

$$
\begin{array}{r}
\left\|\mathscr{R}_{\mathscr{M}}^{(i)} v-v\right\|_{L^{\infty}(\Omega)} \leq \max _{K \in \mathscr{M}}\left\|\mathscr{R}_{\mathscr{M}}^{(i)} v-v\right\|_{L^{\infty}(K)}=\max _{\substack{K=\left[\overrightarrow{\sigma \sigma^{\prime}}\right] \\
\sigma, \sigma^{\prime} \in \mathscr{C}^{(i)}}}\left(\left\|\mathscr{R}_{\mathscr{M}}^{(i)} v-v\right\|_{L^{\infty}\left(D_{\sigma, K}\right)}+\left\|\mathscr{R}_{\mathscr{M}}^{(i)} v-v\right\|_{L^{\infty}\left(D_{\sigma^{\prime}, K}\right)}\right) \\
\leq \frac{1}{2} h_{\mathscr{M}} \sum_{K \in \mathscr{M}}\left\|\partial_{i} v\right\|_{L^{\infty}(K)} \leq c h_{\mathscr{M}}\left\|\partial_{i} v\right\|_{L^{\infty}(\Omega)} .
\end{array}
$$

This completes the proof of estimate (3.67).

Let us now prove (3.66). For the sake of simplicity we assume that $d=2, i=1$ and $j=2$. Other cases are similar. First we write

$$
\mathscr{R}_{\mathscr{E}}^{(i, j)} v-v=\mathscr{R}_{\mathscr{C}}^{(i, j)} v-\mathscr{R}_{\mathscr{M}}^{(i)} v+\mathscr{R}_{\mathscr{M}}^{(i)} v-v
$$

The second term in the right hand side of the previous equality is estimated uisng (3.67). Now using the decomposition of $D_{\sigma}$ established in Figure 7 we can write

$$
\begin{aligned}
& \left\|\mathscr{R}_{\mathscr{C}}^{(i, j)} v-\mathscr{R}_{\mathscr{M}}^{(i)} v\right\|_{L^{p}(\Omega)}^{p}=\sum_{\sigma \in \mathscr{E}(j)}\left\|\mathscr{R}_{\mathscr{C}}^{(i, j)} v-\mathscr{R}_{\mathscr{M}}^{(i)} v\right\|_{L^{p}\left(D_{\sigma}\right)}^{p} \\
& =\sum_{\sigma=K \mid L \in \mathscr{C}_{\text {int }}^{(j)}}\left\|\mathscr{R}_{\mathscr{C}}^{(i, j)} v-\mathscr{R}_{\mathscr{M}}^{(i)} v\right\|_{L^{p}\left(D_{\sigma, K}\right)}^{p}+\left\|\mathscr{R}_{\mathscr{E}}^{(i, j)} v-\mathscr{R}_{\mathscr{M}}^{(i)} v\right\|_{L^{p}\left(D_{\sigma, L}\right)}^{p} \\
& +\sum_{\substack{\sigma \mathscr{C}_{\text {ext }}^{(j)}}}\left\|\mathscr{R}_{\mathscr{E}}^{(i, j)} v-\mathscr{R}_{\mathscr{M}}^{(i)} v\right\|_{L^{p}\left(D_{\sigma}\right)}^{p} \\
& \leq \sum_{\sigma=K \mid L \in \mathscr{E}_{\text {int }}^{(j)}}\left|D _ { \sigma , K } \left\|\left(\mathscr{R}_{\mathscr{G}}^{(i, j)} v\right)_{\sigma}-\left.\left(\mathscr{R}_{\mathscr{M}}^{(i)} v\right)_{K}\right|^{p}+\left|D_{\sigma, L} \|\left(\mathscr{R}_{\mathscr{G}}^{(i, j)} v\right)_{\sigma}-\left(\mathscr{R}_{\mathscr{M}}^{(i)} v\right)_{L}\right|^{p}\right.\right. \\
& +\sum_{\substack{\sigma \mathscr{E}_{\text {ext }}^{(j)} \cap \mathscr{E}(K)}}\left|D_{\sigma} \|\left(\mathscr{R}_{\mathscr{M}}^{(i)} v\right)_{K}\right|^{p}
\end{aligned}
$$

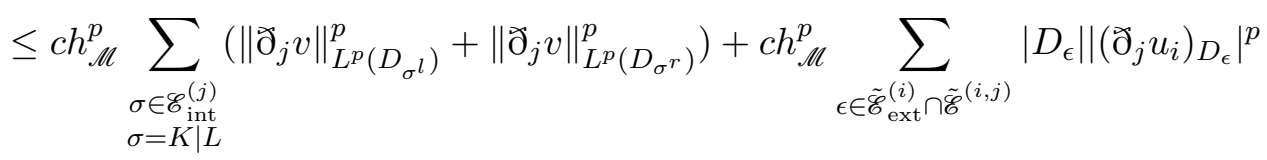

$$
\begin{aligned}
& \leq \operatorname{ch}_{\mathscr{M}}^{p}\left\|ळ_{j} v\right\|_{L^{p}(\Omega)}^{p} .
\end{aligned}
$$

This finishes the proof of Lemma 3.5.

The following algebraic identity is used to transform the terms involving dual fluxes into terms involving primal fluxes, compare with [30, Chapter 3]. It is crucial for the transformation of the discrete convective terms in the proof of the error estimates for the staggered schemes in general, and, in particular, for the MAC scheme.

Lemma 3.6. Let $\varrho \in L_{\mathscr{M}}$ and $\boldsymbol{u} \in \mathbf{H}_{\mathscr{E}, 0}$. Let $i \in\{1, \ldots, d\}$ and let $\varphi=\sum_{\sigma \in \mathscr{E}_{\text {int }}^{(i)}} \varphi_{\sigma} \chi_{D_{\sigma}} \in H_{\mathscr{E}, 0}^{(i)}$ be $a$ discrete scalar function. Let the primal fluxes be given by (3.12) and let the dual fluxes $F_{\epsilon, \sigma}$ be given by (3.19) or (3.20) (depending on the direction of $\mathbf{n}_{\epsilon}$ with respect to $\left.\boldsymbol{e}^{(i)}\right)$. Then we have:

$$
\sum_{\sigma \in \mathscr{C}_{\text {int }}^{(i)}} \sum_{\epsilon \in \widetilde{\mathscr{E}}\left(D_{\sigma}\right)} F_{\epsilon, \sigma} u_{\epsilon} \varphi_{\sigma}=\sum_{K \in \mathscr{M}}\left(\mathscr{R}_{\mathscr{M}}^{(i)} \varphi\right)_{K} \sum_{j=1}^{d} \sum_{\sigma \in \mathscr{E}_{\text {int }}^{(j)}(K)} F_{\sigma, K}\left(\mathscr{R}_{\mathscr{\mathscr { C }}}^{(i, j)} u_{i}\right)_{\sigma}+R^{i}\left(u_{i}, \varphi\right)
$$




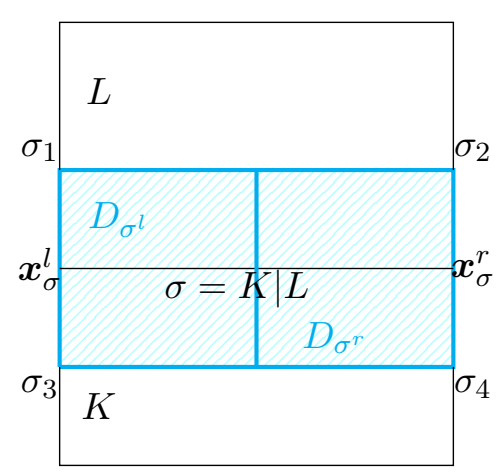

Figure 7: Decomposition of $D_{\sigma}$

where

$$
\begin{aligned}
R^{i}\left(u_{i}, \varphi\right)= & \sum_{K \in \mathscr{M}_{\sigma \in \mathscr{E}_{\text {int }}^{(i)}}} \sum_{K)}\left(\varphi_{\sigma}-\left(\mathscr{R}_{\mathscr{M}}^{(i)} \varphi\right)_{K}\right) F_{\sigma, K}\left(u_{\sigma}-\left(\mathscr{R}_{\mathscr{M}}^{(i)} u_{i}\right)_{K}\right) \\
& +\sum_{K \in \mathscr{M}} \sum_{\sigma \in \mathscr{E}_{\mathrm{int}}^{(i)}(K)}\left(\varphi_{\sigma}-\left(\mathscr{R}_{\mathscr{M}}^{(i)} \varphi\right)_{K}\right) \sum_{j=1, j \neq i}^{d} \sum_{\tau \in \mathscr{E}_{\mathrm{int}}^{(j)}(K)} \sum_{\sigma^{\prime} \in \mathscr{N}_{\tau, \sigma}} \frac{F_{\tau, K}}{2}\left(\frac{u_{i, \sigma}+u_{i, \sigma^{\prime}}}{2}-\left(\mathscr{R}_{\mathscr{M}}^{(i)} u_{i}\right)_{K}\right) .
\end{aligned}
$$

In the last sum we have denoted

$$
\mathcal{N}_{\tau, \sigma}=\left\{\sigma^{\prime} \in \mathscr{E}^{(i)} \mid \operatorname{int}_{d-1} \tau \cap \operatorname{int}_{d-1}\left(D_{\sigma} \mid D_{\sigma^{\prime}}\right) \neq \emptyset\right\},
$$

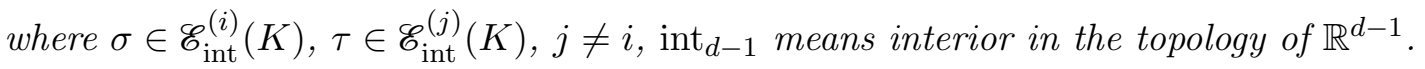

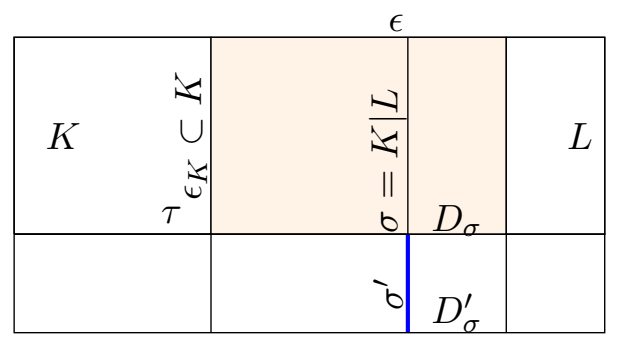

Figure 8: Set $\mathcal{N}_{\tau, \sigma}=\left\{\sigma^{\prime}\right\}$ with $\tau \in \mathscr{E}_{\text {int }}^{(j)}(K), \sigma \in \mathscr{E}^{(i)}(K), j \neq i$ in two dimensions $(i=1, j=2)$

Proof. We split the sum at the left hand side of the identity in Lemma 3.6 to two sums, first one over faces $\epsilon$ parallel to faces $\sigma$ and second one over faces $\epsilon$ orthogonal to sigma:

$$
\begin{aligned}
& \sum_{\sigma \in \mathscr{E}_{\text {int }}^{(i)}} \varphi_{\sigma}\left[\sum_{\epsilon \in \tilde{\mathscr{E}}_{(}\left(D_{\sigma}\right)} F_{\epsilon, \sigma} u_{i, \epsilon}\right]=\sum_{\sigma \in \mathscr{E}_{\text {int }}^{(i)}} \varphi_{\sigma}\left[\sum_{\epsilon \in \tilde{\mathscr{E}}_{\text {int }}\left(D_{\sigma}\right)} F_{\epsilon, \sigma} u_{i, \epsilon}\right] \\
= & \sum_{K \in \mathscr{M}} \sum_{\sigma \in \mathscr{E}_{\text {int }}^{(i)}(K)} \varphi_{\sigma}\left[\sum_{\epsilon \in \tilde{\mathscr{E}}_{\mathrm{int}}\left(D_{\sigma}\right), \epsilon \in K} F_{\epsilon, \sigma} u_{i, \epsilon}+\sum_{j=1, j \neq i} \sum_{\tau \in \mathscr{E}_{\text {int }}^{(j)}} \sum_{\epsilon \in \tilde{\mathcal{N}}_{\tau, \sigma}} \frac{F_{\tau, K}}{2} u_{i, \epsilon}\right],
\end{aligned}
$$

where we have used definition (3.20) of $F_{\epsilon, \sigma}$ and denoted for fixed $\sigma \in \mathscr{E}_{\text {int }}^{(i)}(K)$ and $\tau \in \mathscr{E}_{\text {int }}(K) \backslash \mathscr{E}_{\text {int }}^{(i)}(K)$,

$$
\tilde{\mathcal{N}}_{\tau, \sigma}=\left\{\epsilon \in \tilde{\mathscr{E}}\left(D_{\sigma}\right) \mid \epsilon \perp \sigma, \emptyset \neq \epsilon \cap K \subset \tau\right\} .
$$

For further calculation we notice that (see Figure 9)

$$
\mathcal{N}_{\tau}=\cup_{\sigma \in \mathscr{E}(i)}(K) \mathcal{N}_{\tau, \sigma} \cup \mathscr{E}^{(i)}(K), \quad \tilde{\mathcal{N}}_{\tau, \sigma}=\left\{D_{\sigma}\left|D_{\sigma^{\prime}}\right| \sigma^{\prime} \in \mathcal{N}_{\tau, \sigma}\right\},
$$




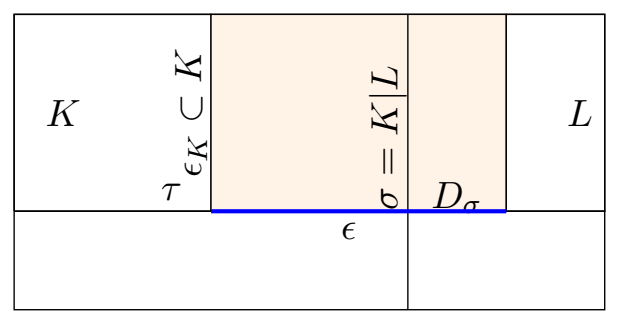

Figure 9: Set $\tilde{\mathcal{N}}_{\tau, \sigma}=\{\epsilon\}$ with $\tau \in \mathscr{E}_{\text {int }}^{(j)}(K), \sigma \in \mathscr{E}^{(i)}(K), j \neq i$ in two dimnsions $(i=1, j=2)$

where $\mathcal{N}_{\tau, \sigma}$ is defined in Lemma 3.6.

Realizing that the set $\left\{\epsilon \in \tilde{\mathscr{E}}\left(D_{\sigma}\right) \mid \epsilon \in K\right\}$ contains exactly one face denoted $\epsilon_{K}$ (see Figure 8), we rewrite the above expression using definitions (3.25), (3.68) as follows

$$
\begin{aligned}
& \sum_{\sigma \in \mathscr{E}_{\text {int }}^{(i)}} \varphi_{\sigma}\left[\sum_{\epsilon \in \widetilde{\mathscr{C}}\left(D_{\sigma}\right)} F_{\epsilon, \sigma} u_{i, \epsilon}\right]=\sum_{K \in \mathscr{M}^{\prime}} \sum_{\sigma \in \mathscr{E}_{\text {int }}^{(i)}(K)} \varphi_{\sigma}\left[F_{\epsilon_{K}, \sigma} u_{i, \epsilon_{K}}+\sum_{j=1, j \neq i}^{d} \sum_{\tau \in \mathscr{E}_{\text {int }}^{(j)}(K)} \sum_{\sigma^{\prime} \in \mathcal{N}_{\tau, \sigma}} \frac{F_{\tau, K}}{2} \frac{u_{i, \sigma}+u_{i, \sigma^{\prime}}}{2}\right] \\
& =\sum_{K \in M^{\prime}} \sum_{\sigma \in \mathscr{C}_{\text {int }}^{(i)}(K)} \varphi_{\sigma}\left[F_{\epsilon_{K}, \sigma} u_{i, \epsilon_{K}}+F_{\sigma, K} u_{i, \sigma}+\sum_{j=1, j \neq i}^{d} \sum_{\tau \in \mathscr{C}_{\mathrm{int}}^{(j)}(K)} \sum_{\sigma^{\prime} \in \mathcal{N}_{\tau, \sigma}} \frac{F_{\tau, K}}{2} \frac{u_{i, \sigma}+u_{i, \sigma^{\prime}}}{2}\right] \\
& =\sum_{K \in \mathscr{M}} \sum_{\sigma \in \mathscr{E}_{\text {int }}^{(i)}(K)}\left(\mathscr{R}_{\mathscr{M}}^{(i)}(\varphi)\right)_{K}\left[F_{\sigma, K} u_{i, \sigma}+\sum_{j=1, j \neq i}^{d} \sum_{\tau \in \mathscr{E}_{\text {int }}^{(j)}(K)} \sum_{\sigma^{\prime} \in \mathcal{N}_{\tau, \sigma}} \frac{F_{\tau, K}}{2} \frac{u_{i, \sigma}+u_{i, \sigma^{\prime}}}{2}\right]
\end{aligned}
$$

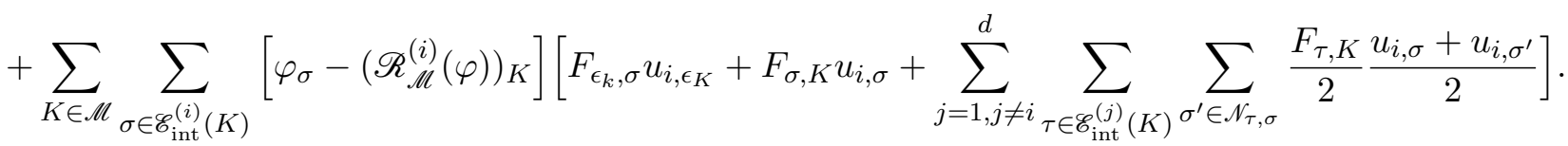

In the above we have used the conservation (3.15) of primal fluxes (which gives in particular the identity $\left.\sum_{K \in \mathscr{M}} \sum_{\sigma \in \mathscr{E}_{\text {int }}^{(i)}(K)} \varphi_{\sigma} F_{\sigma, K} u_{i, \sigma}=0\right)$ to pass from the first to the second expression, and the conservation (3.21) of dual fluxes (in particular $\left.\sum_{K \in \mathscr{M}} \sum_{\sigma \in \mathscr{E}_{\text {int }}^{(i)}(K)}\left(\mathscr{R}_{\mathscr{M}}^{(i)}(\varphi)\right)_{K} F_{\epsilon_{K}, \sigma} u_{i, \epsilon_{K}}=0\right)$. Consequently,

$$
\sum_{K \in \mathscr{M}_{\sigma \in \mathscr{C}_{\text {int }}^{(i)}}} \varphi_{\sigma}\left[\sum_{\epsilon \in \widetilde{\mathscr{C}}\left(D_{\sigma}\right)} F_{\epsilon, \sigma} u_{i, \epsilon}\right]=\sum_{K \in \mathscr{M}}\left(\mathscr{R}_{\mathscr{M}}^{(i)}(\varphi)\right)_{K} I_{i}^{K}+\sum_{K \in \mathscr{M}} J_{i}^{K},
$$

where

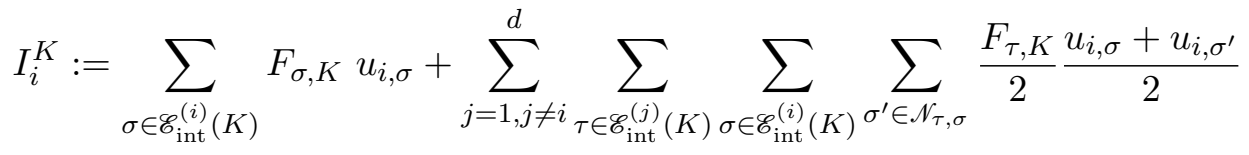

and

$$
J_{i}^{K}:=\sum_{\sigma \in \mathscr{C}_{\mathrm{int}}^{(i)}(K)}\left[\varphi_{\sigma}-\left(\mathscr{R}_{\mathscr{M}}^{(i)}(\varphi)\right)_{K}\right]\left[F_{\epsilon_{k}, \sigma} u_{i, \epsilon_{K}}+F_{\sigma, K} u_{i, \sigma}+\sum_{j=1, j \neq i}^{d} \sum_{\tau \in \mathscr{\mathscr { C }}_{\mathrm{int}}^{(j)}(K)} \sum_{\sigma^{\prime} \in \mathcal{N}_{\tau, \sigma}} \frac{F_{\tau, K}}{2} \frac{u_{i, \sigma}+u_{i, \sigma^{\prime}}}{2}\right] .
$$

Employing (3.68) together with (3.60) (see also Figures 5 and 8) we easily find that

$$
I_{i}^{K}=\sum_{j=1}^{d} \sum_{\sigma \in \mathscr{E}_{\text {int }}^{(j)}(K)} F_{\sigma, K}\left(\mathscr{R}_{\mathscr{E}}^{(i, j)}\left(u_{i}\right)\right)_{\sigma} .
$$

In order to transform $J_{i}^{K}$, we first remark with help of (3.19) the identity

$$
F_{\sigma, K}+F_{\epsilon_{K}, \sigma}+\sum_{j=1, j \neq i}^{d} \sum_{\tau \in \mathscr{E}_{\text {int }}^{(j)}} \sum_{\sigma^{\prime} \in \mathcal{N}_{\tau, \sigma}} \frac{F_{\tau, K}}{2}=\frac{1}{2} \sum_{\sigma \in \mathscr{E}_{\text {int }}(K)} F_{\sigma, K}
$$


consequently,

$$
\begin{gathered}
\sum_{\sigma \in \mathscr{E}_{\text {int }}^{(i)}(K)}\left(\varphi_{\sigma}-\left(\mathscr{R}_{\mathscr{M}}^{(i)} \varphi\right)_{K}\right)\left[F_{\sigma, K}+F_{\epsilon_{K}, \sigma}+\sum_{j=1, j \neq i}^{d} \sum_{\tau \in \mathscr{E}_{\text {int }}^{(j)}(K)} \sum_{\sigma^{\prime} \in \mathscr{N}_{\tau, \sigma}} \frac{F_{\tau, K}}{2}\right]= \\
{\left[\sum_{\sigma \in \mathscr{C}_{\text {int }}(K)} F_{\sigma, K}\right]\left[\left(\sum_{\sigma \in \mathscr{\mathscr { C }}(i)(K)} \frac{\varphi_{\sigma}}{2}\right)-\left(\mathscr{R}_{\mathscr{M}}^{(i)} \varphi\right)_{K}\right]=0,}
\end{gathered}
$$

where, we have used (3.63). Next we write

$$
\begin{aligned}
& J_{i}^{K}=\sum_{\sigma \in \mathscr{E}_{\text {int }}^{(i)}(K)}\left[\varphi_{\sigma}-\left(\mathscr{R}_{\mathscr{M}}^{(i)}(\varphi)\right)_{K}\right]\left[F_{\sigma, K}\left(u_{i, \sigma}-\left(\mathscr{R}_{\mathscr{M}}^{(i)}\left(u_{i}\right)\right)_{K}\right)+F_{\epsilon_{K}, \sigma}\left(u_{i, \epsilon_{K}}-\left(\mathscr{R}_{\mathscr{M}}^{(i)}\left(u_{i}\right)\right)_{K}\right)\right. \\
& \left.+\sum_{j=1, j \neq i}^{d} \sum_{\tau \in \mathscr{E}_{\text {int }}^{(j)}(K)} \sum_{\sigma^{\prime} \in \mathcal{N}_{\tau, \sigma}} \frac{F_{\tau, K}}{2}\left(\frac{u_{i, \sigma}+u_{i, \sigma^{\prime}}}{2}-\left(\mathscr{R}_{\mathscr{M}}^{(i)}\left(u_{i}\right)\right)_{K}\right)\right] \\
& +\sum_{\sigma \in \mathscr{E}_{\text {int }}^{(i)}(K)}\left[\varphi_{\sigma}-\left(\mathscr{R}_{\mathscr{M}}^{(i)}(\varphi)\right)_{K}\right]\left[F_{\epsilon_{k}, \sigma}+F_{\sigma, K}+\sum_{j=1, j \neq i}^{d} \sum_{\tau \in \mathscr{E}_{\text {int }}^{(j)}(K)} \sum_{\sigma^{\prime} \in \mathcal{N}_{\tau, \sigma}} \frac{F_{\tau, K}}{2}\right]\left(\mathscr{R}_{\mathscr{M}}^{(i)}\left(u_{i}\right)\right)_{K},
\end{aligned}
$$

where

$$
u_{i, \epsilon_{K}}-\left(\mathscr{R}_{\mathscr{M}}^{(i)}\left(u_{i}\right)\right)_{K}=0
$$

(due to (3.63) and (3.25)) and

$$
\sum_{\sigma \in \mathscr{C}_{\text {int }}^{(i)}(K)}\left[\varphi_{\sigma}-\left(\mathscr{R}_{\mathscr{M}}^{(i)}(\varphi)\right)_{K}\right]\left[F_{\epsilon_{k}, \sigma}+F_{\sigma, K}+\sum_{j=1, j \neq i}^{d} \sum_{\tau \in \mathscr{C}_{\text {int }}^{(j)}} \sum_{\sigma^{\prime} \in \mathcal{N}_{\tau, \sigma}} \frac{F_{\tau, K}}{2}\right]\left(\mathscr{R}_{\mathscr{M}}^{(i)}\left(u_{i}\right)\right)_{K}=0
$$

due to (3.71). Consequently,

$$
\begin{aligned}
& R^{i}\left(u_{i}, \varphi\right)=\sum_{K \in \mathscr{M}} J_{i}^{K}=\sum_{K \in \mathscr{M}_{\sigma \in \mathscr{E}_{\text {int }}^{(i)}(K)}}\left(\varphi_{\sigma}-\left(\mathscr{R}_{\mathscr{M}}^{(i)} \varphi\right)_{K}\right) F_{\sigma, K}\left(u_{\sigma}-\left(\mathscr{R}_{\mathscr{M}^{\prime}}^{(i)} u_{i}\right)_{K}\right) \\
& \quad+\sum_{K \in \mathscr{M}} \sum_{\sigma \in \mathscr{C}_{\text {int }}^{(i)}(K)}\left(\varphi_{\sigma}-\left(\mathscr{R}_{\mathscr{M}}^{(i)} \varphi\right)_{K}\right) \sum_{j=1, j \neq i}^{d} \sum_{\tau \in \mathscr{C}_{\text {int }}^{(j)}(K)} \sum_{\sigma^{\prime} \in \mathscr{N}_{\tau, \sigma}} \frac{F_{\tau, K}}{2}\left(\frac{u_{i, \sigma}+u_{i, \sigma^{\prime}}}{2}-\left(\mathscr{R}_{\mathscr{M}^{(i)}}^{\left.\left.\left(u_{i}\right)\right)_{K}\right)}\right.\right.
\end{aligned}
$$

Putting together formulas (3.69), (3.70) and (3.72) concludes the proof of Lemma 3.6.

\subsection{Main result: error estimates}

Now, we are ready to state the main result of this paper. For the sake of clarity, we shall state the theorem and perform the proofs only in the most interesting three dimensional case. The modifications to be done for the two dimensional case, which is in fact more simple, are mostly due to the different Sobolev embedings and are left to the interested reader.

Let us introduce the following functional space:

$$
\begin{array}{r}
\mathscr{F}=\left\{(r, \boldsymbol{U}) \in C^{1}([0, T] \times \bar{\Omega})^{4}, 0<\underline{r}=\inf _{(t, x) \in \bar{Q}_{T}} r(t, x), \nabla^{2} \boldsymbol{U} \in C([0, T] \times \bar{\Omega})^{3}, \partial_{t}^{2} r \in L^{1}\left(0, T ; L^{\gamma^{\prime}}(\Omega)\right),\right. \\
\left.\partial_{t} \nabla r \in L^{2}\left(0, T ; L^{6 \gamma /(5 \gamma-6)}(\Omega)^{3}\right),\left(\partial_{t}^{2} \boldsymbol{U}, \partial_{t} \nabla \boldsymbol{U}\right) \in L^{2}\left(0, T ; L^{6 / 5}(\Omega)^{12}\right)\right\}, \quad(3.73)
\end{array}
$$

endowed with the following norm

$$
\begin{array}{r}
\|(r, \boldsymbol{U})\|_{\mathscr{F}}=\|(r, \boldsymbol{U})\|_{C^{1}([0, T] \times \bar{\Omega})^{4}}+\left\|\nabla^{2} \boldsymbol{U}\right\|_{C([0, T] \times \bar{\Omega})^{3}}+\left\|\partial_{t}^{2} r\right\|_{L^{1}\left(0, T ; L^{\prime}(\Omega)\right)}+\left\|\partial_{t} \nabla r\right\|_{L^{2}\left(0, T ; L^{\left.6 \gamma /(5 \gamma-6)(\Omega)^{3}\right)}\right.} \\
+\left\|\partial_{t}^{2} \boldsymbol{U}\right\|_{L^{2}\left(0, T ; L^{6 / 5}(\Omega)^{12}\right)}+\left\|\partial_{t} \nabla \boldsymbol{U}\right\|_{L^{2}\left(0, T ; L^{6 / 5}(\Omega)^{12}\right)} .
\end{array}
$$


Let $(r, \boldsymbol{U}) \in \mathscr{F}$ such that $\boldsymbol{U}=\mathbf{0}$ on $\partial \Omega$. Let us consider a MAC grid $\mathscr{D}=(\mathscr{M}, \mathscr{E})$ of size $h_{\mathscr{M}}$ and regularity $\eta_{\mathscr{M}}$ of the computational domain $\Omega$, a partition $0=t^{0}<t^{1}<\ldots<t^{N}=T$ of the time interval $[0, T]$, which, for the sake of simplicity, we suppose uniform (where $\delta t$ stands for the constant time step) and $(\varrho, \boldsymbol{u}) \in Y_{\mathscr{M}, \delta t} \times \boldsymbol{X}_{\mathscr{E}, \delta t}$ a solution of the discrete problem (3.10). Inspired by (2.10), we introduce the discrete relative energy functional

$$
\begin{aligned}
\mathfrak{E}\left(\varrho^{n}, \boldsymbol{u}^{n} \mid r_{\mathscr{M}}^{n}, \boldsymbol{U}_{\mathscr{E}}^{n}\right) & =\int_{\Omega}\left(\frac{1}{2} \varrho^{n}\left|\boldsymbol{u}^{n}-\boldsymbol{U}_{\mathscr{E}}^{n}\right|^{2}+E\left(\varrho^{n} \mid r_{\mathscr{M}}^{n}\right)\right) \mathrm{d} \boldsymbol{x} \\
& =\sum_{i=1}^{3} \sum_{\sigma \in \mathscr{E}_{\mathrm{int}}^{(i)}} \frac{1}{2}\left|D_{\sigma}\right| \varrho_{D_{\sigma}}^{n}\left|u_{\sigma}^{n}-U_{\sigma}^{n}\right|^{2}+\sum_{K \in \mathscr{M}}|K| E\left(\varrho_{K}^{n} \mid r_{K}^{n}\right),
\end{aligned}
$$

where

$$
r^{n}=r\left(t^{n}, \cdot\right), \boldsymbol{U}^{n}=\boldsymbol{U}\left(t^{n}, \cdot\right), r_{\mathscr{M}}^{n}=\mathscr{P}_{\mathscr{M}}\left(r^{n}\right), \boldsymbol{U}_{\mathscr{E}}^{n}=\mathscr{P}_{\mathscr{E}}\left(\boldsymbol{U}^{n}\right),
$$

where $\mathscr{P}_{\mathscr{M}}$ and $\mathscr{P}_{\mathscr{C}}$ are respectively defined in (3.41) and (3.46). Finally as in Proposition 2.1 we denote

$$
0<\underline{r}=\min _{(0, T) \times \Omega} r, \quad \bar{r}=\max _{(0, T) \times \Omega} r, \quad\left[\partial_{t} r\right]^{n}=\partial_{t} r\left(t^{n}, \cdot\right) .
$$

Let us now state that the discrete problem (3.10) admits at least one solution. This existence result follows from standard arguments of the topological degree theory (see [8] for the theory, [10] for the first application to a nonlinear scheme). We refer to Appendix A for its proof. The theorem reads:

Theorem 3.1. Let $\left(\varrho^{0}, \boldsymbol{u}^{0}\right) \in L_{\mathscr{M}} \times \mathbf{H}_{\mathscr{C}, 0}$ such that $\varrho^{0}>0$ (that is $\varrho_{K}^{0}>0$ for any $K \in \mathscr{M}$ ). There exists a solution $(\boldsymbol{u}, \varrho) \in \mathbf{H}_{\mathscr{E}, 0} \times L_{\mathscr{M}}$ of Problem (3.10). Moreover any solution is such that $\varrho>0$ a.e in $\Omega$ (meaning that $\varrho_{K}^{n}>0$ for any $n=1, \ldots, N$ and for any $K \in \mathscr{M}$ ). (2.18)

The following Theorem is the main result of the paper. It can be seen as a discrete version of inequality

Theorem 3.2 (Error estimate). Let $\Omega \subset \mathbb{R}^{3}$ be a domain which is a union of orthogonal closed parallelepipeds with mutually disjoint interiors, and, without loss of generality, such that the faces of these parallelepipeds are orthogonal to the canonical basis vectors. Assume that the viscosity coefficients satisfy assumptions (1.4) and that the pressure p satisfy (1.5) where $\gamma>\frac{3}{2}$. Let $\mathscr{D}=(\mathscr{M}, \mathscr{E})$ be a MAC grid of $\Omega$ (see Definition 3.1 in Section 3), with step size $h_{\mathscr{M}}$ (see (3.6)) and regularity $\eta_{\mathscr{M}}$ where $\eta_{\mathscr{M}}$ is defined in (3.8). Let us consider a partition $0=t^{0}<t^{1}<\ldots<t^{N}=T$ of the time interval $[0, T]$, which, for the sake of simplicity, we suppose uniform, where $\delta t$ stands for the constant time step. Let $(\varrho, \boldsymbol{u}) \in Y_{\mathscr{M}, \delta t} \times \boldsymbol{X}_{\mathscr{E}, \delta t}$ be a solution of the discrete problem (3.10) emanating from $\left(\varrho^{0}, \boldsymbol{u}^{0}\right) \in L_{\mathscr{M}} \times \mathbf{H}_{\mathscr{E}, 0}$ such that $\varrho^{0}>0$ (the existence of which granted by Theorem 3.1), and $(r, \boldsymbol{U}) \in \mathscr{F}$ be a (strong) solution of problem (1.1). Then there exists a constant $c>0$ only depending on $T,|\Omega|, p_{0}, p_{\infty}, \mu, \lambda, \gamma, \underline{r}, \min _{[\underline{r}, \bar{r}]} p, \min _{[\underline{r} / 2,2 \bar{r}]} p^{\prime},\|p\|_{C^{2}([\underline{r}, \bar{r}])}$, $\|(r, \boldsymbol{U})\|_{\mathscr{F}}$, on $\mathscr{E}_{0, \mathscr{M}}$ in a nondecreasing way and on $\eta_{\mathscr{M}}$ in a nondecreasing way such that

$$
\max _{0 \leq n \leq N} \mathfrak{E}\left(\varrho^{n}, \boldsymbol{u}^{n} \mid r_{\mathscr{M}}^{n}, \boldsymbol{U}_{\mathscr{E}}^{n}\right) \leq c\left(\mathfrak{E}\left(\varrho^{0}, \boldsymbol{u}^{0} \mid r_{\mathscr{M}}^{0}, \boldsymbol{U}_{\mathscr{E}}^{0}\right)+h_{\mathscr{M}}^{A}+\sqrt{\delta t}\right)
$$

where

$$
A=\min \left(\frac{2 \gamma-3}{\gamma}, \frac{1}{2}\right)
$$

\section{Remark 2.}

1. As mentioned previously, Theorem 3.2 holds also in dimension 2 under the assumption that $\gamma>1$. The value of $A$ in the error estimate (3.78) is

$$
\left\{\begin{array}{l}
A<\min \left(\frac{2 \gamma-2}{\gamma}, \frac{1}{2}\right) \text { if } \gamma \in\left(1, \frac{4}{3}\right], \\
A=\frac{1}{2} \text { if } \gamma>\frac{4}{3} .
\end{array}\right.
$$


2. Suppose that the discrete initial data $\left(\varrho^{0}, \boldsymbol{u}^{0}\right)$ coincides with the projection $\left(\mathscr{P}_{\mathscr{M}} r_{0}, \mathscr{P}_{\mathscr{C}} \boldsymbol{u}_{0}\right)$ of the initial data determining the strong solution. Then formula (3.78) (combined with Lemma 2.1 and with the fact that $\mathscr{E}_{0, \mathscr{M}}$ is bounded uniformly with respect to the mesh) provides, in terms of classical Lebesgue spaces, the following bounds:

$$
\left\|\varrho^{n}-r^{n}\right\|_{L^{2}\left(\left\{\underline{r} / 2 \leq \varrho^{n} \leq 2 \bar{r}\right\}\right)}^{2}+\left\|\boldsymbol{u}^{n}-\boldsymbol{U}^{n}\right\|_{L^{2}\left(\left\{\underline{r} / 2 \leq \varrho^{n} \leq 2 \bar{r}\right\}\right)}^{2} \leq c\left(h_{\mathscr{M}}^{A}+\sqrt{\delta t}\right)
$$

for the "essential part" of the solution (where the numerical density remains bounded from above and from below outside zero), and

$$
\left|\left\{\varrho^{n} \leq \underline{r} / 2\right\}\right|+\left|\left\{\varrho^{n} \geq 2 \bar{r}\right\}\right|+\left\|\varrho^{n}\right\|_{L^{\gamma}\left(\Omega \cap\left\{\varrho^{n} \geq 2 \bar{r}\right\}\right)}^{\gamma}+\left\|\varrho^{n}\left|\boldsymbol{u}^{n}-\boldsymbol{U}^{n}\right|^{2}\right\|_{L^{1}\left(\left\{\varrho^{n} \geq 2 \bar{r}\right\}\right)} \leq c\left(h_{\mathscr{M}^{A}}^{A}+\sqrt{\delta t}\right)
$$

for the "residual part" of the solution, where the numerical density can be "close" to zero or infinity. (In the above formula, for $B \subset \Omega,|B|$ denotes the Lebesgue measure of B.) In particular, we obtain

$$
\|\varrho-r\|_{L^{2}(\{\underline{r} / 2 \leq \varrho \leq 2 \bar{r}\})}^{2}+\|\boldsymbol{u}-\boldsymbol{U}\|_{L^{2}(\{\underline{r} / 2 \leq \varrho \leq 2 \bar{r}\})}^{2} \leq c\left(h_{\mathscr{M}}^{A}+\sqrt{\delta t}\right) .
$$

Moreover, in the particular case of $p(\varrho)=\varrho^{2}$ (that however represents a non physical situation) $E(\varrho \mid r)=(\varrho-r)^{2}$ and the error estimate (3.78) gives

$$
\left\|\varrho^{n}-r^{n}\right\|_{L^{\infty}\left(0, T ; L^{2}(\Omega)\right)}^{2}+\left\|\varrho^{n}\left|\boldsymbol{u}^{n}-\boldsymbol{U}^{n}\right|^{2}\right\|_{L^{\infty}\left(0, T ; L^{1}(\Omega)\right)} \leq c\left(\sqrt{h}_{\mathscr{M}}+\sqrt{\delta t}\right)
$$

3. If we assume that the discrete density $\varrho$ is bounded from above uniformly with respect to $\left(h_{\mathscr{M}}, \delta t\right)$, the growth condition at infinity in (1.5) becomes irrelevant. In this case, following step by step proof of Theorem 3.2 we obtain error estimate (3.78) with $A=\frac{1}{2}$ for any $\gamma \geq 1$. Compare with [14], where the similar problem is treated for a Finite volume/Finite element method. This is qualitatively better result than any other conditional error estimate in the mathematical literature dealing with finite volume or mixed finite volume/finite element methods for compressible fluids (see [4], [10], [34], [44], [48]), where the authors need to assume other bounds for the numerical solution, in addition to the upper bound for the density.

4. Theorem 3.2 can be viewed as a discrete version of Proposition 2.1. It is to be noticed that the assumptions on the constitutive law for pressure guaranteeing the error estimates for the scheme (3.10) are somewhat stronger $(\gamma \geq 3 / 2)$ than the assumptions needed for the stability in the continuous case $\left(\gamma>\frac{6}{5}\right)$. In particular, the asymptotic behaviour of the pressure for small densities is not needed in the continuous case. The threshold value $\gamma=3 / 2$ is however in accordance with the existence theory of weak solutions. The assumptions on the regularity of the strong solution to be compared with the discrete solution in the scheme are slightly stronger than those needed to establish the stability estimates in the continuous case.

5. The assumption on the asymptotic behaviour of the pressure for small densities in (1.5) can be relaxed for $\gamma \geq 2$, see [24]. In particular Theorem 3.2 also holds for the isentropic pressure law $p(\varrho)=\varrho^{\gamma}$ where $\gamma \geq 2$.

The rest of the paper is devoted to the proof of Theorem 3.2. We employ the methodology inspired by that one suggested in [15] in the continuous case. It can be summarized as follows:

1. We establish the energy identity for discrete solutions of the numerical scheme - see Theorem 4.1, formula (4.1). This corresponds to the energy inequality (2.6) in the continuous case.

2. Knowing (4.1) we establish the discrete relative energy inequality for the discrete solution of the numerical scheme with test functions taken in the discrete spaces introduced in Definition 3.2 - see formula (5.1) in Theorem 5.1. This is a numerical counterpart of relative energy inequality (2.11) in the continuous case. 
3. Now, we take first in the discrete relative energy inequality as test functions the projections $\left(r_{\mathscr{M}}, \boldsymbol{U}_{\mathscr{E}}\right)$ of a smooth couple $(r, \boldsymbol{U})$ and transform conveniently each term at its right hand side, see formula (5.14) in Lemma 5.1. Then we take in the discrete relative energy inequality as test functions the projections $\left(r_{\mathscr{M}}, \boldsymbol{U}_{\mathscr{E}}\right)$ of a strong solution $(r, \boldsymbol{U})$ to the problem (1.1-1.3), in order to derive a consistency error for the strong solution, see equality (6.1) in Lemma 6.1. We must do it in such a way that the leading expressions in all terms at the right hand side of formulas (5.1) and (6.1) are 'comparable', provided $(r, \boldsymbol{U})$ in both inequalities is a regular solution of problem (1.1-1.3). In contrast with the error estimate [24] for the academic scheme [36], this process for the MAC scheme (and for the staggered schemes, in general) is more involved and far to be obvious. The difficulties are caused by the fact that the defects of 'structure' of MAC discretization with respect to the structure of the problem on the continuous level are more important than the same defects of the academic scheme [36]. The most difficult terms in this process are the convective terms. It is here, where Lemma 3.6 plays crucial role.

4. Combining Lemma 5.1 and Lemma 6.1 we obtain inequality (7.1). This inequality is a numerical counterpart of relative energy inequality (2.12) in the continuous case.

5. We estimate conveniently the right hand side of inequality (7.1) in order to get the Gronwall type estimate, see Lemma 7.1. The latter Lemma implies the result.

\section{Mesh independent estimates}

\subsection{Energy Inequality}

Our analysis starts with an energy equality (which can be seen as a discrete differential version of (2.6)), which is crucial both in the convergence analysis and in the error analysis.

Theorem 4.1 (Energy estimate). Let $(\varrho, \boldsymbol{u}) \in Y_{\mathscr{M}, \delta t} \times \boldsymbol{X}_{\mathscr{E}, \delta t}$ be a solution of (3.10). Then for any $n=1, \ldots, N$, there exists $\varrho^{n-1, n} \in L_{\mathscr{M}}$ such that $\min \left(\varrho^{n-1}, \varrho^{n}\right) \leq \varrho^{n-1, n} \leq \max \left(\varrho^{n-1}, \varrho^{n}\right)$ and $\varrho_{\sigma}^{n} \in$ $\left[\min \left(\varrho_{K}^{n}, \varrho_{L}^{n}\right), \max \left(\varrho_{K}^{n}, \varrho_{L}^{n}\right)\right], \sigma=K \mid L \in \mathscr{E}_{\text {int }}$ such that

$$
\begin{aligned}
\frac{1}{\delta t} \int_{\Omega} \mathscr{H}\left(\varrho^{n}\right)- & \mathscr{H}\left(\varrho^{n-1}\right) \mathrm{d} \boldsymbol{x}+\frac{1}{2 \delta t} \int_{\Omega} \varrho^{n}\left|\boldsymbol{u}^{n}\right|^{2}-\varrho^{n-1}\left|\boldsymbol{u}^{n-1}\right|^{2} \mathrm{~d} \boldsymbol{x} \\
& +\mu\left\|\boldsymbol{u}^{n}\right\|_{1, \mathscr{E}, 0}^{2}+(\mu+\lambda)|| \operatorname{div} \mathscr{M} \boldsymbol{u}^{n}||_{L^{2}(\Omega)}^{2}+\frac{1}{2 \delta t} \int_{\Omega} \varrho^{n-1}\left|\boldsymbol{u}^{n}-\boldsymbol{u}^{n-1}\right|^{2} \mathrm{~d} \boldsymbol{x} \\
& +\int_{\Omega} \frac{1}{2 \delta t} \mathscr{H}^{\prime \prime}\left(\varrho^{n-1, n}\right)\left(\varrho^{n}-\varrho^{n-1}\right)^{2} \mathrm{~d} \boldsymbol{x}+\frac{1}{2} \sum_{\sigma \in \mathscr{E}_{\text {int }}, \sigma=K \mid L}|\sigma| \mathscr{H}^{\prime \prime}\left(\varrho_{\sigma}^{n}\right)\left(\varrho_{K}^{n}-\varrho_{L}^{n}\right)^{2}\left|\boldsymbol{u}_{\sigma, K}^{n}\right|=0 .
\end{aligned}
$$

The notation and all discrete operators employed in formula (4.1) are introduced in Section 3.

Proof. Multiplying (3.10a) by $\mathscr{H}^{\prime}\left(\varrho^{n}\right)$ and using a Taylor expansion we obtain the existence of $\varrho^{n-1, n} \in$ $L_{\mathscr{M}}$ such that $\min \left(\varrho^{n-1}, \varrho^{n}\right) \leq \varrho^{n-1, n} \leq \max \left(\varrho^{n-1}, \varrho^{n}\right)$ and

$$
\int_{\Omega} \frac{\mathscr{H}\left(\varrho^{n}\right)-\mathscr{H}^{\prime}\left(\varrho^{n-1}\right)}{\delta t} \mathrm{~d} \boldsymbol{x}+\int_{\Omega} \frac{1}{2 \delta t} \mathscr{H}^{\prime \prime}\left(\varrho^{n-1, n}\right)\left(\varrho^{n}-\varrho^{n-1}\right)^{2} \mathrm{~d} \boldsymbol{x}+\int_{\Omega} \operatorname{div}_{\mathscr{M}}^{\mathrm{up}}\left(\varrho^{n} \boldsymbol{u}^{n}\right) \mathscr{H}^{\prime}\left(\varrho^{n}\right) \mathrm{d} \boldsymbol{x}=0
$$

Using again a Taylor expansion (see for instance [21]) one has

$$
\int_{\Omega} \operatorname{div}_{\mathscr{M}}^{\text {up }}\left(\varrho^{n} \boldsymbol{u}^{n}\right) \mathscr{H}^{\prime}\left(\varrho^{n}\right) \mathrm{d} \boldsymbol{x}=\int_{\Omega} p\left(\varrho^{n}\right) \operatorname{div} \mathscr{M} \boldsymbol{u}^{n} \mathrm{~d} \boldsymbol{x}+\frac{1}{2} \sum_{\sigma \in \mathscr{E}_{\text {int }}, \sigma=K \mid L}|\sigma| \mathscr{H}^{\prime \prime}\left(\varrho_{\sigma}^{n}\right)\left(\varrho_{K}^{n}-\varrho_{L}^{n}\right)^{2}\left|\boldsymbol{u}_{\sigma, K}^{n}\right|
$$

where $\varrho_{\sigma}^{n} \in\left[\min \left(\varrho_{K}^{n}, \varrho_{L}^{n}\right), \max \left(\varrho_{K}^{n}, \varrho_{L}^{n}\right)\right]$. Consequently

$$
\begin{aligned}
\int_{\Omega} \frac{\mathscr{H}\left(\varrho^{n}\right)-\mathscr{H}\left(\varrho^{n-1}\right)}{\delta t} \mathrm{~d} \boldsymbol{x} & +\int_{\Omega} \frac{1}{2 \delta t} \mathscr{H}^{\prime \prime}\left(\varrho^{n-1, n}\right)\left(\varrho^{n}-\varrho^{n-1}\right)^{2} \mathrm{~d} \boldsymbol{x} \\
& +\int_{\Omega} p\left(\varrho^{n}\right) \operatorname{div} \mathscr{M}^{n} \mathrm{~d} \boldsymbol{x}+\frac{1}{2} \sum_{\sigma \in \mathscr{E}_{\text {int }}, \sigma=K \mid L}|\sigma| \mathscr{H}^{\prime \prime}\left(\varrho_{\sigma}^{n}\right)\left(\varrho_{K}^{n}-\varrho_{L}^{n}\right)^{2}\left|\boldsymbol{u}_{\sigma, K}^{n}\right|=0 .
\end{aligned}
$$


Mutliplying (3.10b) by $u_{\sigma}^{n}$, summing over $\sigma \in \mathscr{E}_{\text {int }}^{(i)}$ and $i=1,2,3$ and using (3.31) we infer that

$$
\begin{aligned}
\int_{\Omega} \frac{\varrho^{n} \boldsymbol{u}^{n}-\varrho^{n-1} \boldsymbol{u}^{n-1}}{\delta t} \cdot \boldsymbol{u}^{n} \mathrm{~d} \boldsymbol{x}+\mu\left\|\boldsymbol{u}^{n}\right\|_{1, \mathscr{C}, 0}^{2}+(\mu+\lambda)\left\|\operatorname{div} \boldsymbol{M}^{n}\right\|_{L^{2}(\Omega)}^{2} \\
\quad+\sum_{i=1}^{3} \sum_{\sigma \in \mathscr{E}_{\text {int }}^{(i)}} \sum_{\epsilon \in \tilde{\mathscr{E}}\left(D_{\sigma}\right)} F_{\epsilon, \sigma}^{n} u_{\epsilon}^{n} u_{\sigma}^{n}-\int_{\Omega} p\left(\varrho^{n}\right) \operatorname{div} \mathscr{M} \boldsymbol{u}^{n} \mathrm{~d} \boldsymbol{x}=0 .
\end{aligned}
$$

By virtue of the centered choice for $u_{\epsilon}^{n}$ (see (3.25)) we have

$$
\sum_{i=1}^{3} \sum_{\sigma \in \mathscr{\mathscr { C }}_{\text {int }}^{(i)}} \sum_{\epsilon \in \tilde{\mathscr{E}}\left(D_{\sigma}\right)} F_{\epsilon, \sigma}^{n} u_{\epsilon}^{n} u_{\sigma}^{n}=\sum_{i=1}^{3} \sum_{\sigma \in \mathscr{\mathscr { C }}_{\text {int }}^{(i)}} \sum_{\epsilon \in \tilde{\mathscr{E}}\left(D_{\sigma}\right)} F_{\epsilon, \sigma}^{n} \frac{\left(u_{\sigma}^{n}\right)^{2}}{2} .
$$

Multiplying (3.24) by $\frac{\left(u_{\sigma}^{n}\right)^{2}}{2}$ and summing over $\sigma \in \mathscr{E}_{\text {int }}^{(i)}$ and $i=1,2,3$ we infer that

$$
\frac{1}{2 \delta t} \int_{\Omega}\left(\varrho^{n}-\varrho^{n-1}\right)\left|\boldsymbol{u}^{n}\right|^{2} \mathrm{~d} \boldsymbol{x}+\sum_{i=1}^{3} \sum_{\sigma \in \mathscr{E}_{\text {int }}^{(i)}} \sum_{\epsilon \in \tilde{\mathscr{E}}\left(D_{\sigma}\right)} F_{\epsilon, \sigma}^{n} \frac{\left(u_{\sigma}^{n}\right)^{2}}{2}=0
$$

Subtracting (4.7) and (4.5) gives

$$
\begin{aligned}
\frac{1}{2 \delta t} \int_{\Omega} \varrho^{n}\left|\boldsymbol{u}^{n}\right|^{2}-\varrho^{n-1}\left|\boldsymbol{u}^{n-1}\right|^{2} \mathrm{~d} \boldsymbol{x}+\mu & \left\|\boldsymbol{u}^{n}\right\|_{1, \mathscr{C}, 0}^{2}+(\mu+\lambda)\left\|\operatorname{div} \mathscr{M} \boldsymbol{u}^{n}\right\|_{L^{2}(\Omega)}^{2} \\
& +\frac{1}{2 \delta t} \int_{\Omega} \varrho^{n-1}\left|\boldsymbol{u}^{n}-\boldsymbol{u}^{n-1}\right|^{2} \mathrm{~d} \boldsymbol{x}-\int_{\Omega} p\left(\varrho^{n}\right) \operatorname{div}_{\mathscr{M}} \boldsymbol{u}^{n} \mathrm{~d} \boldsymbol{x}=0 .
\end{aligned}
$$

Consequently adding (4.4) to (4.8) together with (4.3) yields (4.1).

Remark 3. Theorem 4.1 shows that this numerical scheme is unconditionally stable, meaning that the discrete energy inequality holds without any extra assumptions on the discrete solution.

The following estimates are obtained thanks to the identity (4.1) and Lemma 3.2. In particular the numerical diffusion (4.16) is bounded due to the upwind discretization of convective terms and assumptions (1.5), as is classical in the framework of the hyperbolic conservation laws, see e.g. [11].

Corollary 4.1. Let $(\varrho, \boldsymbol{u}) \in Y_{\mathscr{M}, \delta t} \times \boldsymbol{X}_{\mathscr{E}, \delta t}$ be a solution of (3.10). Then we have

1. There exists $c>0$ only depending on $\mathscr{E}_{0, \mathscr{M}}$ in a nondecreasing way (independent of $h_{\mathscr{M}}$ and $\delta t$ ) such that

$$
\begin{gathered}
\|\boldsymbol{u}\|_{L^{2}\left(0, T ; \mathbf{H}_{\mathscr{\delta}, 0}(\Omega)\right)} \leq c, \\
\|\boldsymbol{u}\|_{L^{2}\left(0, T ; L^{6}(\Omega)^{3}\right)} \leq c, \\
\left\|\varrho|\boldsymbol{u}|^{2}\right\|_{L^{\infty}\left(0, T ; L^{1}(\Omega)\right)} \leq c, \\
\|\varrho\|_{L^{\infty}\left(0, T ; L^{\gamma}(\Omega)\right)} \leq c, \\
\|\varrho \boldsymbol{u}\|_{L^{2}\left(0, T ; L^{\left.\frac{6 \gamma}{\gamma+6}(\Omega)^{3}\right)}\right.} \leq c .
\end{gathered}
$$

2. If $(r, \boldsymbol{U}) \in L^{\infty}((0, T) \times \Omega) \times L^{\infty}((0, T) \times \Omega)^{3}$, then

$$
\max _{1 \leq n \leq N} \mathfrak{E}\left(\varrho^{n}, \boldsymbol{u}^{n} \mid r_{\mathscr{M}}^{n}, \boldsymbol{U}_{\mathscr{E}}^{n}\right) \leq c,
$$

where $c$ depends on $\bar{r},\|\boldsymbol{U}\|_{L^{\infty}((0, T) \times \Omega)^{3}}, \mathscr{E}_{0, \mathscr{M}}$ in a nondecreasing way and the projections $r_{\mathscr{M}}$ and $\boldsymbol{U}_{\mathscr{E}}$ are defined in (3.76). 
3. There exists $c$ only depending on $\mathscr{E}_{0, \mathscr{M}}$ in a nondecreasing way such that for any $m=1, \ldots, N$

$$
\sum_{n=1}^{m} \sum_{i=1}^{3} \sum_{\sigma \in \mathscr{C}_{\text {int }}^{(i)}}\left|D_{\sigma}\right| \varrho_{D_{\sigma}}^{n-1}\left|u_{\sigma}^{n}-u_{\sigma}^{n-1}\right|^{2} \leq c .
$$

4. The following dissipation estimate due to the upwinding of the density in (3.10a) and (1.5) holds

$$
\begin{aligned}
\delta t \sum_{n=1}^{N} \sum_{\sigma=K \mid L \in \mathscr{E}_{\text {int }}}|\sigma| \frac{\left(\varrho_{K}^{n}-\varrho_{L}^{n}\right)^{2}}{\left[\max \left(\varrho_{K}^{n}, \varrho_{L}^{n}\right)\right]^{(2-\gamma)^{+}}} 1_{\left\{\varrho_{\sigma}^{n} \geq 1\right\}}\left|u_{\sigma, K}^{n}\right| \\
\quad+\delta t \sum_{n=1}^{N} \sum_{\sigma=K \mid L \in \mathscr{E}_{\text {int }}}|\sigma|\left(\varrho_{K}^{n}-\varrho_{L}^{n}\right)^{2} 1_{\left\{\varrho_{\sigma}^{n}<1\right\}}\left|u_{\sigma, K}^{n}\right| \leq c .
\end{aligned}
$$

where $c$ depends on $\mathscr{E}_{0, \mathcal{M}}$ in a nondecreasing way and where the quantity $\bar{\varrho}_{\sigma}^{n}$ is defined in Theorem 4.1. In the above the symbol $1_{\{s \geq 1\}}$ is equal to 1 if $s \geq 1$ and 0 if $s<1$, while $1_{\{s<1\}}$ is equal to 1 if $s<1$ and 0 otherwise.

\section{Relative energy inequality for the discrete problem}

\subsection{Exact relative energy inequality for the discrete problem}

The goal of this section is to prove the discrete (differential) version of the relative energy inequality (2.11).

Theorem 5.1 (Exact discrete relative energy). Any solution $(\varrho, \boldsymbol{u}) \in Y_{\mathscr{M}, \delta t} \times \boldsymbol{X}_{\mathscr{E}, \delta t}$ of the discrete problem (3.10) satisfy

$$
\begin{gathered}
\frac{1}{\delta t}\left(\mathfrak{E}\left(\varrho^{n}, \boldsymbol{u}^{n} \mid r^{n}, \boldsymbol{U}^{n}\right)-\mathfrak{E}\left(\varrho^{n-1}, \boldsymbol{u}^{n-1} \mid r^{n-1}, \boldsymbol{U}^{n-1}\right)\right)+\mu\left\|\boldsymbol{u}^{n}-\boldsymbol{U}^{n}\right\|_{1, \mathscr{E}, 0}^{2}+(\mu+\lambda)\left\|\operatorname{div}_{\mathscr{M}}\left(\boldsymbol{u}^{n}-\boldsymbol{U}^{n}\right)\right\|_{L^{2}(\Omega)}^{2} \\
\leq \int_{\Omega}\left(r^{n}-\varrho^{n}\right) \frac{\mathscr{H}^{\prime}\left(r^{n}\right)-\mathscr{H}^{\prime}\left(r^{n-1}\right)}{\delta t} \mathrm{~d} \boldsymbol{x}+\int_{\Omega} \operatorname{div}_{\mathscr{M}}^{\mathrm{up}}\left(\varrho^{n} \boldsymbol{u}^{n}\right) \mathscr{H}^{\prime}\left(r^{n-1}\right) \mathrm{d} \boldsymbol{x} \\
\quad+\mu\left[\boldsymbol{U}^{n}-\boldsymbol{u}^{n}, \boldsymbol{U}^{n}\right]_{1, \mathscr{E}, 0}+(\mu+\lambda) \int_{\Omega} \operatorname{div}_{\mathscr{M}}\left(\boldsymbol{U}^{n}-\boldsymbol{u}^{n}\right) \operatorname{div}_{\mathscr{M}} \boldsymbol{U}^{n} \mathrm{~d} \boldsymbol{x} \\
\quad-\int_{\Omega} p\left(\varrho^{n}\right) \operatorname{div}_{\mathscr{M}} \boldsymbol{U}^{n} \mathrm{~d} \boldsymbol{x}+\int_{\Omega} \varrho^{n-1} \frac{\boldsymbol{U}^{n-1}-\boldsymbol{U}^{n}}{\delta t} \cdot\left(\boldsymbol{u}^{n-1}-\frac{1}{2}\left(\boldsymbol{U}^{n-1}+\boldsymbol{U}^{n}\right)\right) \mathrm{d} \boldsymbol{x} \\
\quad+\sum_{i=1}^{3} \sum_{\sigma \in \mathscr{E}_{\mathrm{int}}^{(i)}} \sum_{\epsilon \in \widetilde{\mathscr{E}}\left(D_{\sigma}\right)} F_{\epsilon, \sigma}^{n} U_{\sigma}^{n}\left(u_{\epsilon}^{n}-U_{\epsilon}^{n}\right) \quad(5.1)
\end{gathered}
$$

for any $0<r \in Y_{\mathscr{M}, \delta t}, \boldsymbol{U} \in \boldsymbol{X}_{\mathscr{C}, \delta t}$.

The notation and all discrete operators employed in formula (5.1) are introduced in Section 3.

We notice, comparing the terms in the "discrete" formula (5.1) with the terms in the "continuous" formula (2.11), that Theorem 5.1 represents a discrete counterpart of the "continuous" relative energy inequality (2.11). The rest of this section is devoted to its proof. To this end, we shall follow the proof of the "continuous" relative energy inequality (see [15] and [19]) and adapt it to the discrete case.

Proof. We proceed in several steps.

Investigation of the momentum equation (3.10b) : Multiplying (3.10b) by $\boldsymbol{U}^{n}$ and integrating over $\Omega$ we obtain

$$
\begin{aligned}
\int_{\Omega} \frac{1}{\delta t}\left(\varrho^{n} \boldsymbol{u}^{n}-\varrho^{n-1} \boldsymbol{u}^{n-1}\right) & \cdot \boldsymbol{U}^{n} \mathrm{~d} \boldsymbol{x}+\sum_{i=1}^{3} \sum_{\sigma \in \mathscr{E}_{\text {int }}^{(i)}} \sum_{\epsilon \in \widetilde{\mathscr{E}}\left(D_{\sigma}\right)} F_{\epsilon, \sigma}^{n} u_{\epsilon}^{n} U_{\sigma}^{n} \\
& +\mu\left[\boldsymbol{u}^{n}, \boldsymbol{U}^{n}\right]_{1, \mathscr{\ell}, 0}+(\mu+\lambda) \int_{\Omega} \operatorname{div}_{\mathscr{M}} \boldsymbol{u}^{n} \operatorname{div}_{\mathscr{M}} \boldsymbol{U}^{n} \mathrm{~d} \boldsymbol{x}-\int_{\Omega} p^{n} \operatorname{div}_{\mathscr{M}} \boldsymbol{U}^{n} \mathrm{~d} \boldsymbol{x}=0
\end{aligned}
$$


We observe that

$$
\left(\varrho^{n} \boldsymbol{u}^{n}-\varrho^{n-1} \boldsymbol{u}^{n-1}\right) \cdot \boldsymbol{U}^{n}=\varrho^{n} \boldsymbol{u}^{n} \cdot U^{n}-\varrho^{n-1} \boldsymbol{u}^{n-1} \cdot \boldsymbol{U}^{n-1}+\varrho^{n-1} \boldsymbol{u}^{n-1} \cdot\left(\boldsymbol{U}^{n-1}-\boldsymbol{U}^{n}\right) .
$$

Consequently

$$
\begin{array}{r}
-\frac{1}{\delta t} \int_{\Omega} \varrho^{n} \boldsymbol{u}^{n} \cdot \boldsymbol{U}^{n}-\varrho^{n-1} \boldsymbol{u}^{n-1} \cdot \boldsymbol{U}^{n-1} \mathrm{~d} \boldsymbol{x}=\frac{1}{\delta t} \int_{\Omega} \varrho^{n-1} \boldsymbol{u}^{n-1} \cdot\left(\boldsymbol{U}^{n-1}-\boldsymbol{U}^{n}\right) \mathrm{d} \boldsymbol{x}+\sum_{i=1}^{3} \sum_{\sigma \in \mathscr{\mathscr { C }}(i)} \sum_{\epsilon \in \widetilde{\mathscr{E}}\left(D_{\sigma}\right)} F_{\epsilon, \sigma}^{n} u_{\epsilon}^{n} U_{\sigma}^{n} \\
+\mu\left[\boldsymbol{u}^{n}, \boldsymbol{U}^{n}\right]_{1, \mathscr{C}, 0}+(\mu+\lambda) \int_{\Omega} \operatorname{div} \mathscr{M}^{n} \boldsymbol{u}^{n} \operatorname{div}_{\mathscr{M}} \boldsymbol{U}^{n} \mathrm{~d} \boldsymbol{x}-\int_{\Omega} p^{n} \operatorname{div}_{\mathscr{M}} \boldsymbol{U}^{n} \mathrm{~d} \boldsymbol{x} . \quad(5.2)
\end{array}
$$

Investigation of the dual continuity equation (3.24) : Multiplying (3.24) by $\frac{1}{2}\left|U_{\sigma}^{n}\right|^{2}$ we obtain

$$
\frac{1}{2 \delta t} \int_{\Omega}\left(\varrho^{n}-\varrho^{n-1}\right)\left|\boldsymbol{U}^{n}\right|^{2} \mathrm{~d} \boldsymbol{x}+\frac{1}{2} \sum_{i=1}^{3} \sum_{\sigma \in \mathscr{E}_{\text {int }}^{(i)}} \sum_{\epsilon \in \widetilde{\mathscr{C}}\left(D_{\sigma}\right)} F_{\epsilon, \sigma}^{n}\left|U_{\sigma}^{n}\right|^{2}=0 .
$$

Moreover due to (3.21)

$$
\frac{1}{2} \sum_{i=1}^{3} \sum_{\sigma \in \mathscr{C}_{\text {int }}^{(i)}} \sum_{\epsilon \in \widetilde{\mathscr{C}}\left(D_{\sigma}\right)} F_{\epsilon, \sigma}^{n} U_{\sigma}^{n} U_{\sigma^{\prime}}^{n}=0
$$

We observe that

$$
\int_{\Omega}\left(\varrho^{n}-\varrho^{n-1}\right)\left|\boldsymbol{U}^{n}\right|^{2} \mathrm{~d} \boldsymbol{x}=\int_{\Omega} \varrho^{n}\left|\boldsymbol{U}^{n}\right|^{2}-\varrho^{n-1}\left|\boldsymbol{U}^{n-1}\right|^{2} \mathrm{~d} \boldsymbol{x}+\int_{\Omega} \varrho^{n-1}\left(\boldsymbol{U}^{n-1}+\boldsymbol{U}^{n}\right) \cdot\left(\boldsymbol{U}^{n-1}-\boldsymbol{U}^{n}\right) \mathrm{d} \boldsymbol{x} .
$$

which gives

$$
\begin{array}{r}
\int_{\Omega} \frac{1}{2 \delta t}\left(\varrho^{n}\left|\boldsymbol{U}^{n}\right|^{2}-\varrho^{n-1}\left|\boldsymbol{U}^{n-1}\right|^{2}\right) \mathrm{d} \boldsymbol{x}=-\frac{1}{2 \delta t} \int_{\Omega} \varrho^{n-1}\left(\boldsymbol{U}^{n-1}+\boldsymbol{U}^{n}\right) \cdot\left(\boldsymbol{U}^{n-1}-\boldsymbol{U}^{n}\right) \mathrm{d} \boldsymbol{x} \\
-\frac{1}{2} \sum_{i=1}^{3} \sum_{\sigma \in \mathscr{E}_{\text {int }}^{(i)}} \sum_{\epsilon \in \widetilde{\mathscr{E}}\left(D_{\sigma}\right)} F_{\epsilon, \sigma}^{n}\left|U_{\sigma}^{n}\right|^{2} .
\end{array}
$$

Investigation of the primal continuity equation (3.10a) : Multiplying the continuity equation by $\mathscr{H}^{\prime}\left(r^{n-1}\right)$ and integrating over $\Omega$ we obtain

$-\frac{1}{\delta t} \int_{\Omega}\left(\varrho^{n} \mathscr{H}^{\prime}\left(r^{n}\right)-\varrho^{n-1} \mathscr{H}^{\prime}\left(r^{n-1}\right)\right) \mathrm{d} \boldsymbol{x}=-\frac{1}{\delta t} \int_{\Omega} \varrho^{n}\left(\mathscr{H}^{\prime}\left(r^{n}\right)-\mathscr{H}^{\prime}\left(r^{n-1}\right)\right) \mathrm{d} \boldsymbol{x}+\int_{\Omega} \operatorname{div}_{\mathscr{M}}^{\mathrm{up}}\left(\varrho^{n} \boldsymbol{u}^{n}\right) \mathscr{H}^{\prime}\left(r^{n-1}\right) \mathrm{d} \boldsymbol{x}$.

Finally, thanks to the the convexity of the function $\mathscr{H}$, we have

$$
\begin{array}{r}
\frac{1}{\delta t} \int_{\Omega}\left[\left(r^{n} \mathscr{H}^{\prime}\left(r^{n}\right)-\mathscr{H}\left(r^{n}\right)\right)-\left(r^{n-1} \mathscr{H}^{\prime}\left(r^{n-1}\right)-\mathscr{H}\left(r^{n-1}\right)\right)\right] \mathrm{d} \boldsymbol{x}=\frac{1}{\delta t} \int_{\Omega} r^{n}\left(\mathscr{H}^{\prime}\left(r^{n}\right)-\mathscr{H}^{\prime}\left(r^{n-1}\right)\right) \mathrm{d} \boldsymbol{x} \\
-\frac{1}{\delta t} \int_{\Omega}\left(\mathscr{H}\left(r^{n}\right)-\left(r^{n}-r^{n-1}\right) \mathscr{H}^{\prime}\left(r^{n-1}\right)-\mathscr{H}\left(r^{n-1}\right)\right) \mathrm{d} \boldsymbol{x} \\
\leq \frac{1}{\delta t} \int_{\Omega} r^{n}\left(\mathscr{H}^{\prime}\left(r^{n}\right)-\mathscr{H}^{\prime}\left(r^{n-1}\right)\right) \mathrm{d} \boldsymbol{x} . \quad(5.7)
\end{array}
$$

Conclusion : Summing (4.1), (5.2), (5.4), (5.5), (5.6) and (5.7) we obtain (5.1).

\subsection{Approximate relative energy inequality for the discrete problem}

The exact relative energy inequality as stated in Section 5.1 is a general inequality for the given numerical scheme, however it does not immediately provide a comparison of the approximate solution with the strong solution of the compressible Navier-Stokes equations. Its right hand side has to be conveniently 
transformed (modulo the possible appearance of residual terms vanishing as the space and time steps tend to 0) to provide such comparison tool via a Gronwall type argument.

The goal of this section is to derive a version of the discrete relative energy inequality, still with arbitrary (sufficiently regular) test functions $(r, \boldsymbol{U})$, that will be convenient for the comparison of the discrete solution with the strong solution.

Let us introduce some notations useful for the rest of the paper. Considering a solution $(\varrho, \boldsymbol{u})$ of Problem 3.10, and $(r, \boldsymbol{U}) \in \mathscr{F}$ we define for $\sigma=K \mid L \in \mathscr{E}_{\text {int }}$ :

$$
r_{\sigma}^{n, \text { up }}=\mid \begin{array}{ll}
r_{K}^{n} & \text { if } u_{\sigma, K}^{n} \geq 0, \\
r_{L}^{n} & \text { otherwise }
\end{array}
$$

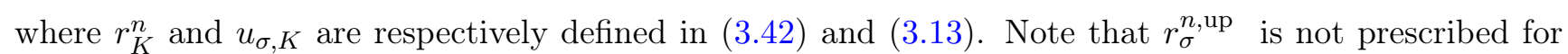
$\sigma \in \mathscr{E}_{\text {ext }}$ (it is a consequence of the fact that $u_{\sigma, K}$ vanishes for $\sigma \in \mathscr{E}(K) \cap \mathscr{E}_{\text {ext }}$ ). Similarly to (3.23) we define

$$
\begin{array}{ll}
\text { for } \sigma \in \mathscr{E}_{\text {int }}, \sigma=K \mid L & \left|D_{\sigma}\right| r_{D_{\sigma}}^{n}=\left|D_{\sigma, K}\right| r_{K}^{n}+\left|D_{\sigma, L}\right| r_{L}^{n}, \\
\text { for } \sigma \in \mathscr{E}_{\text {ext }}, \sigma \in \mathscr{E}(K), & r_{D_{\sigma}}^{n}=r_{K}^{n} .
\end{array}
$$

For $i=1, \ldots, d$ and $\epsilon=D_{\sigma} \mid D_{\sigma^{\prime}} \in \tilde{\mathscr{E}}_{\text {int }}^{(i)}$ we set

$$
U_{\epsilon}^{n}=\frac{\left(\mathscr{P}_{\mathscr{E}}^{(i)} U_{i}^{n}\right)_{\sigma}+\left(\mathscr{P}_{\mathscr{E}}^{(i)} U_{i}^{n}\right)_{\sigma^{\prime}}}{2}
$$

where $\mathscr{P}_{\mathscr{E}}^{(i)}$ is defined in $(3.44)$.

Starting from now, we shall use the following convention for the constants in estimates. We shall denote by $c$ a positive number which can take different values even in the same formula. It always depend tacitly on the geometric and structural coefficients

$$
T,|\Omega|, p_{0}, p_{\infty}, \mu, \lambda, \gamma,
$$

and if not stated explicitly otherwise, on the characteristics of the strong solution

$$
\underline{r}, \min _{[\underline{r}, \bar{r}]} p, \min _{[\underline{[} / 2,2 \bar{r}]} p^{\prime},\|p\|_{C^{2}([\underline{r}, \bar{r}])},\|(r, \boldsymbol{U})\|_{\mathscr{F}}
$$

and on

$$
\mathscr{E}_{0, \mathscr{M}} \text { in a non decreasing way, } \eta_{\mathscr{M}} \text { in a nondecreasing way. }
$$

It is always independent of the size of the discretisation $\delta t$ and $h_{\mathscr{M}}$.

Lemma 5.1 (Approximate discrete relative energy). Let $(\varrho, \boldsymbol{u}) \in Y_{\mathscr{M}, \delta t} \times \boldsymbol{X}_{\mathscr{C}, \delta t}$ be a solution of the discrete problem (3.10) and $(r, \boldsymbol{U}) \in \mathscr{F}$ such that $\boldsymbol{U}_{\mid \partial \Omega}=\mathbf{0}$. Then there exists c only depending on parameters (5.11-5.13) such that for all $m=1, \ldots, N$ :

$$
\begin{aligned}
& \mathfrak{E}\left(\varrho^{m}, \boldsymbol{u}^{m} \mid r_{\mathscr{M}}^{m}, \boldsymbol{U}_{\mathscr{E}}^{m}\right)-\mathfrak{E}\left(\varrho^{0}, \boldsymbol{u}^{0} \mid r_{\mathscr{M}}^{0}, \boldsymbol{U}_{\mathscr{E}}^{0}\right) \\
& +\delta t \sum_{n=1}^{m}\left(\mu\left\|\boldsymbol{u}^{n}-\boldsymbol{U}_{\mathscr{E}}^{n}\right\|_{1, \mathscr{E}, 0}^{2}+(\mu+\lambda)\left\|\operatorname{div}_{\mathscr{M}}\left(\boldsymbol{u}^{n}-\boldsymbol{U}_{\mathscr{E}}^{n}\right)\right\|_{L^{2}(\Omega)}^{2}\right) \\
& \leq \delta t \sum_{n=1}^{m}\left(\mu\left[\boldsymbol{U}_{\mathscr{E}}^{n}-\boldsymbol{u}^{n}, \boldsymbol{U}_{\mathscr{E}}^{n}\right]_{1, \mathscr{E}, 0}+(\mu+\lambda) \int_{\Omega} \operatorname{div}_{\mathscr{M}}\left(\boldsymbol{U}_{\mathscr{E}}^{n}-\boldsymbol{u}^{n}\right) \operatorname{div}_{\mathscr{M}} \boldsymbol{U}_{\mathscr{E}}^{n} \mathrm{~d} \boldsymbol{x}\right) \\
& +\delta t \sum_{n=1}^{m} \int_{\Omega} \varrho^{n-1}\left(\frac{\boldsymbol{U}_{\mathscr{E}}^{n}-\boldsymbol{U}_{\mathscr{E}}^{n-1}}{\delta t}\right) \cdot\left(\boldsymbol{U}_{\mathscr{E}}^{n}-\boldsymbol{u}^{n}\right)+\delta t \sum_{n=1}^{m} \int_{\Omega}\left(r_{\mathscr{M}}^{n}-\varrho^{n}\right) \frac{p^{\prime}\left(r_{\mathscr{M}}^{n}\right)}{r_{\mathscr{M}}^{n}}\left[\partial_{t} r\right]^{n} \mathrm{~d} \boldsymbol{x} \\
& +\delta t \sum_{n=1}^{m} \sum_{i=1}^{3} \sum_{K \in \mathscr{M}} \sum_{j=1}^{3} \sum_{\sigma \in \mathscr{C}^{(j)}(K)}|\sigma| \varrho_{\sigma}^{n, \mathrm{up}}\left(\mathscr{P}_{\mathscr{E}}^{(j)} U_{j}^{n}\right)_{\sigma} \boldsymbol{e}^{(j)} \cdot \boldsymbol{n}_{\sigma, K} \\
& \times\left(\mathscr{R}_{\mathscr{E}}^{(i, j)}\left(u_{i}^{n}-\mathscr{P}_{\mathscr{E}}^{(i)} U_{i}^{n}\right)\right)_{\sigma}\left(\left(\mathscr{R}_{\mathscr{M}}^{(i)}\left(\mathscr{P}_{\mathscr{E}}^{(i)} U_{i}^{n}\right)\right)_{K}-\left(\mathscr{P}_{\mathscr{E}}^{(j)} U_{i}^{n}\right)_{\sigma}\right) \\
& -\delta t \sum_{n=1}^{m} \int_{\Omega} \frac{\varrho^{n}}{r_{\mathscr{M}}^{n}} p^{\prime}\left(r_{\mathscr{M}}^{n}\right) \mathscr{R}_{\mathscr{M}}\left(\boldsymbol{u}^{n}\right) \cdot \nabla r^{n} \mathrm{~d} \boldsymbol{x}-\delta t \sum_{n=1}^{m} \int_{\Omega} p\left(\varrho^{n}\right) \operatorname{div} \boldsymbol{U}^{n} \mathrm{~d} \boldsymbol{x}+\mathscr{R}_{\mathscr{M}, \delta t}^{m}+\mathscr{G}_{\mathscr{M}, \delta t}^{m}
\end{aligned}
$$


for any pair $(r, \boldsymbol{U})$ belonging to the class (3.73) such that $\boldsymbol{U}_{\mid \partial \Omega}=\mathbf{0}$, where

$$
\left|\mathscr{G}_{\mathscr{M}, \delta t}^{m}\right| \leq \frac{c}{\delta} \delta t \sum_{n=1}^{m} \mathfrak{E}\left(\varrho^{m}, \boldsymbol{u}^{m} \mid r_{\mathscr{M}}^{m}, \boldsymbol{U}_{\mathscr{E}}^{m}\right)+\delta \delta t \sum_{n=1}^{m}\left\|\boldsymbol{u}^{n}-\boldsymbol{U}_{\mathscr{E}}^{n}\right\|_{1, \mathscr{C}, 0}^{2},
$$

with any $\delta>0$,

$$
\left|\mathscr{R}_{\mathscr{M}, \delta t}^{m}\right| \leq c\left(\sqrt{\delta t}+h_{\mathscr{M}}^{A}\right)
$$

and where $A$ is given by (3.79).

The notation and all discrete operators employed in formula (5.14) are introduced in Section 3.

Proof. The right hand side of the relative energy inequality (5.1), after a summation over $n$ and a multiplication by $\delta t$, is a sum $\sum_{i=1}^{6} T_{i}$, where

$$
\begin{aligned}
& T_{1}=\delta t \sum_{n=1}^{m}\left(\mu\left[\boldsymbol{U}_{\mathscr{E}}^{n}, \boldsymbol{U}_{\mathscr{E}}^{n}-\boldsymbol{u}^{n}\right]_{1, \mathscr{E}, 0}+(\mu+\lambda) \int_{\Omega} \operatorname{div}_{\mathscr{M}} \boldsymbol{U}_{\mathscr{E}}^{n} \operatorname{div}_{\mathscr{M}}\left(\boldsymbol{U}_{\mathscr{E}}^{n}-\boldsymbol{u}^{n}\right) \mathrm{d} \boldsymbol{x}\right), \\
& T_{2}=\delta t \sum_{n=1}^{m} \int_{\Omega} \varrho^{n-1} \frac{\boldsymbol{U}_{\mathscr{E}}^{n}-\boldsymbol{U}_{\mathscr{E}}^{n-1}}{\delta t} \cdot\left(\frac{\boldsymbol{U}_{\mathscr{E}}^{n-1}+\boldsymbol{U}_{\mathscr{E}}^{n}}{2}-\boldsymbol{u}^{n-1}\right) \mathrm{d} \boldsymbol{x},
\end{aligned}
$$

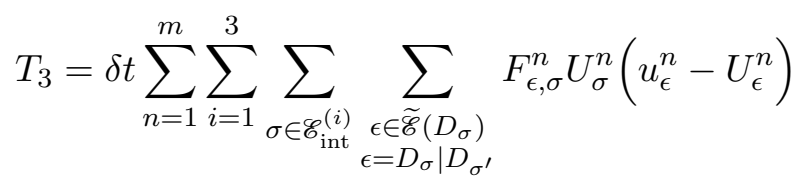

$$
\begin{aligned}
& T_{4}=-\delta t \sum_{n=1}^{m} \int_{\Omega} p\left(\varrho^{n}\right) \operatorname{div} \boldsymbol{U}^{n} \mathrm{~d} \boldsymbol{x}, \\
& T_{5}=\delta t \sum_{n=1}^{m} \int_{\Omega}\left(r_{\mathscr{M}}^{n}-\varrho^{n}\right) \frac{\mathscr{H}^{\prime}\left(r_{\mathscr{M}}^{n}\right)-\mathscr{H}^{\prime}\left(r_{\mathscr{M}}^{n-1}\right)}{\delta t} \mathrm{~d} \boldsymbol{x} \\
& T_{6}=\delta t \sum_{n=1}^{m} \int_{\Omega} \operatorname{div}_{\mathscr{M}}^{\mathrm{up}}\left(\varrho^{n} \boldsymbol{u}^{n}\right) \mathscr{H}^{\prime}\left(r_{\mathscr{M}}^{n-1}\right) \mathrm{d} \boldsymbol{x}
\end{aligned}
$$

The term $T_{1}$ and $T_{4}$ will be kept as they are; all the other terms $T_{i}$ will be transformed to a more convenient form, as described in the following steps.

Step 1: Term $T_{2}$. We have $T_{2}=T_{2,1}+R_{2,1}$ with

$$
\left\{\begin{array}{l}
T_{2,1}=\delta t \sum_{n=1}^{m} \int_{\Omega} \varrho^{n-1}\left(\frac{\boldsymbol{U}_{\mathscr{\mathscr { C }}}^{n}-\boldsymbol{U}_{\mathscr{\mathscr { C }}}^{n-1}}{\delta t}\right) \cdot\left(\boldsymbol{U}_{\mathscr{\mathscr { C }}}^{n-1}-\boldsymbol{u}^{n-1}\right) \mathrm{d} \boldsymbol{x}, \\
R_{2,1}=\sum_{n=1}^{m} \int_{\Omega} \frac{1}{2} \varrho^{n-1}\left|U_{\mathscr{E}}^{n}-U_{\mathscr{E}}^{n-1}\right|^{2} \mathrm{~d} \boldsymbol{x} .
\end{array}\right.
$$

Thanks to the mass conservation (3.16), (4.12) and the Taylor formula applied to the function $t \rightarrow \boldsymbol{U}(t, \boldsymbol{x})$ between $t^{n-1}$ and $t^{n}$ we easily get

$$
\left|R_{2,1}\right| \leq c \delta t
$$

where $c$ depends on $\left\|\partial_{t} \boldsymbol{U}\right\|_{L^{\infty}([0, T] \times \bar{\Omega})^{3}}$ and on $\mathscr{E}_{0, \mathfrak{M}}$. Let us now decompose the term $T_{2,1}$ as

$$
T_{2,1}=T_{2,2}+R_{2,2}, \text { with } T_{2,2}=\delta t \sum_{n=1}^{m} \int_{\Omega} \varrho^{n-1}\left(\frac{\boldsymbol{U}_{\mathscr{E}}^{n}-\boldsymbol{U}_{\mathscr{E}}^{n-1}}{\delta t}\right) \cdot\left(\boldsymbol{U}_{\mathscr{E}}^{n}-\boldsymbol{u}^{n}\right) \mathrm{d} \boldsymbol{x}
$$

and $R_{2,2}=\delta t \sum_{n=1}^{m} R_{2,2}^{n}$ where

$$
R_{2,2}^{n}=\int_{\Omega} \varrho^{n-1}\left(\frac{\boldsymbol{U}_{\mathscr{E}}^{n}-\boldsymbol{U}_{\mathscr{E}}^{n-1}}{\delta t}\right) \cdot\left(\boldsymbol{U}_{\mathscr{E}}^{n-1}-\boldsymbol{U}_{\mathscr{E}}^{n}\right) \mathrm{d} \boldsymbol{x}-\int_{\Omega} \varrho^{n-1}\left(\frac{\boldsymbol{U}_{\mathscr{E}}^{n}-\boldsymbol{U}_{\mathscr{E}}^{n-1}}{\delta t}\right) \cdot\left(\boldsymbol{u}^{n-1}-\boldsymbol{u}^{n}\right) \mathrm{d} \boldsymbol{x} .
$$

By the same token as above, and using estimate (4.15) we may estimate the residual term as follows

$$
\left|R_{2,2}\right| \leq c \sqrt{\delta t}
$$


where $c$ depends on $\left\|\partial_{t} \boldsymbol{U}\right\|_{L^{\infty}((0, T) \times \Omega)^{3}}$ and on $\mathscr{E}_{0, \mathscr{M}}$.

Step 2: Term $T_{3}$. Using Lemma 3.6 we can write

$$
\begin{array}{r}
T_{3}=\delta t \sum_{n=1}^{m} \sum_{i=1}^{3} \sum_{K \in \mathscr{M}}\left(\mathscr{R}_{\mathscr{M}}^{(i)} \mathscr{P}_{\mathscr{E}}^{(i)} U_{i}^{n}\right)_{K} \sum_{j=1}^{3} \sum_{\sigma \in \mathscr{E}_{\text {int }}^{(j)}(K)}|\sigma| \varrho_{\sigma}^{n, \text { up }} u_{\sigma, K}^{n}\left(\mathscr{R}_{\mathscr{E}}^{(i, j)}\left(u_{i}^{n}-\mathscr{P}_{\mathscr{E}}^{(i)} U_{i}^{n}\right)\right)_{\sigma} \\
+\delta t \sum_{n=1}^{m} \sum_{i=1}^{3}\left(R_{3,1,1, i}^{n}+R_{3,1,2, i}^{n}\right)=T_{3,1}+R_{3,1}
\end{array}
$$

where the reminder $R_{3,1, i}^{n}$ and $R_{3,2, i}^{n}$ are respectively given by

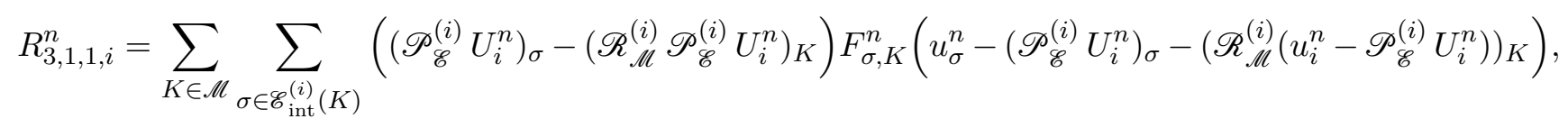

and

$$
\begin{aligned}
& R_{3,1,2, i}^{n}=\sum_{K \in \mathscr{M}_{\sigma \in \mathscr{E}_{\mathrm{int}}^{(i)}}(K)}\left(\left(\mathscr{P}_{\mathscr{E}}^{(i)} U_{i}^{n}\right)_{\sigma}-\left(\mathscr{R}_{\mathscr{M}}^{(i)} \mathscr{P}_{\mathscr{E}}^{(i)} U_{i}^{n}\right)_{K}\right) \\
& \times \sum_{j=1, j \neq i}^{d} \sum_{\tau \in \mathscr{E}_{\text {int }}^{(j)}(K)} \sum_{\sigma^{\prime} \in \mathcal{N}_{\tau, \sigma}} \frac{F_{\tau, K}^{n}}{2}\left(\frac{u_{i, \sigma}^{n}+u_{i, \sigma^{\prime}}^{n}-\left(\mathscr{P}_{\mathscr{E}}^{(i)} U_{i}^{n}\right)_{\sigma}-\left(\mathscr{P}_{\mathscr{E}}^{(i)} U_{i}^{n}\right)_{\sigma^{\prime}}}{2}-\left(\mathscr{R}_{\mathscr{M}}^{(i)}\left(u_{i}^{n}-\mathscr{P}_{\mathscr{E}}^{(i)} U_{i}^{n}\right)\right)_{K}\right) .
\end{aligned}
$$

From the definition of $\mathscr{R}_{\mathscr{M}}^{(i)}$ and $\mathscr{P}_{\mathscr{E}}^{(i)}$, cf. (3.41), (3.44), we infer that

$$
R_{3,1,1, i}^{n}=\sum_{K \in \mathscr{M}_{\sigma \in \mathscr{E}_{\text {int }}^{(i)}}} \sum_{\frac{1}{4}} \frac{|K|^{2}}{|\sigma|^{2}}\left({\mathrm{\partial}_{i}}_{i}\left(\mathscr{P}_{\mathscr{\mathscr { g }}}^{(i)} U_{i}^{n}\right)\right)_{K}\left(\check{\partial}_{i}\left(u_{i}-\mathscr{P}_{\mathscr{E}}^{(i)} U_{i}^{n}\right)\right)_{K} F_{\sigma, K}^{n}
$$

From (3.49) and the definition (3.28) of the discrete derivative, we infer that for any $i \in\{1,2,3\}$ and $K \in \mathscr{M}$

$$
\left|\left(\varlimsup_{i}\left(\mathscr{P}_{\mathscr{E}}^{(i)} U_{i}^{n}\right)\right)_{K}\right| \leq c,
$$

where $c$ depends on $\eta_{\mathscr{M}}$ and on $\|\nabla \boldsymbol{U}\|_{L^{\infty}((0, T) \times \Omega)^{3 \times 3}}$. Using the Hölder's inequality we infer that

$$
\begin{aligned}
& \left|R_{3,1,1, i}^{n}\right| \leq c \sum_{\sigma \in \mathscr{E}_{\text {int }}^{(i)}, \sigma=K \mid L} \sqrt{h_{\sigma}}|\sigma| \varrho_{D_{\sigma}}^{n}\left|u_{\sigma}^{n}\right|\left\|\partial_{i}\left(u_{i}^{n}-\mathscr{P}_{\mathscr{E}}^{(i)}\left(U_{i}^{n}\right)\right)\right\|_{L^{2}(K \cup L)} \\
& \leq c \sum_{\sigma \in \mathscr{E}_{\text {int }}^{(i)}, \sigma=K \mid L} \sqrt{h_{\sigma}}|\sigma|\left|D_{\sigma}\right|^{-\frac{\gamma+6}{6 \gamma}}\left\|\oiint_{i}\left(u_{i}^{n}-\mathscr{P}_{\mathscr{E}}^{(i)}\left(U_{i}^{n}\right)\right)\right\|_{L^{2}(K \cup L)}\left\|\varrho^{n} u_{i}^{n}\right\|_{L^{\frac{6 \gamma}{\gamma+6}\left(D_{\sigma}\right)}},
\end{aligned}
$$

where $c$ depends on $\|\nabla \boldsymbol{U}\|_{L^{\infty}((0, T) \times \Omega)^{3 \times 3}}$ and on $\eta_{\mathscr{M}}$. We can write for all $\sigma \in \mathscr{E}_{\text {int }}^{(i)}$,

$$
c_{1} h_{\sigma}^{3} \leq\left|D_{\sigma}\right| \leq c_{2} h_{\sigma}^{3}, \quad c_{3} h_{\sigma}^{2} \leq|\sigma| \leq c_{4} h_{\sigma}^{2}
$$

, where $c_{1}$ and $c_{3}$ depend on $\eta_{\mathscr{M}}$ in a nondecreasing way and $c_{2}$ and $c_{4}$ depend on $\eta_{\mathscr{M}}$ in a nondecreasing way, which gives

$$
\sqrt{h_{\sigma}}|\sigma|\left|D_{\sigma}\right|^{-\frac{\gamma+6}{6 \gamma}} \leq c h_{M}^{A},
$$

where $c$ depends on $\eta_{\mathscr{M}}$ and where $A$ is given by (3.79). Consequently

$$
\begin{gathered}
\left|R_{3,1,1, i}^{n}\right| \leq \operatorname{ch}_{\mathscr{M}}^{A} \sum_{\sigma \in \mathscr{C}_{\text {int }}^{(i)}, \sigma=K \mid L}\left\|\oiint_{i}\left(u_{i}^{n}-\mathscr{P}_{\mathscr{E}}^{(i)}\left(U_{i}^{n}\right)\right)\right\|_{L^{2}(K \cup L)}\left\|\varrho^{n} u_{i}^{n}\right\|_{L^{\frac{6 \gamma}{\gamma+6}\left(D_{\sigma}\right)}} . \\
\left.\left|R_{3,1,1, i}^{n}\right| \leq \operatorname{ch} \operatorname{M}_{\mathscr{M}}^{A}\left(\sum_{\sigma \in \mathscr{E}_{\text {int }}^{(i)}, \sigma=K \mid L}\left\|\oiint_{i}\left(u_{i}^{n}-\mathscr{P}_{\mathscr{E}}^{(i)}\left(U_{i}^{n}\right)\right)\right\|_{L^{2}(K \cup L)}^{p}\right)^{\frac{1}{p}}\left(\sum_{\sigma \in \mathscr{E}_{\text {int }}^{(i)}, \sigma=K \mid L}\left\|\varrho^{n} u_{i}^{n}\right\|_{L^{\frac{6 \gamma}{\gamma+6}\left(D_{\sigma}\right)}}^{q}\right)\right)^{\frac{1}{q}} .
\end{gathered}
$$


where $1<p, q<+\infty$ and $\frac{1}{p}+\frac{1}{q}=1$.

If $\frac{3}{2} \leq \gamma \leq 3$, then $\frac{6 \gamma}{5 \gamma-6} \geq 2$ and we take $p=\frac{6 \gamma}{5 \gamma-6}, q=\frac{6 \gamma}{\gamma+6}$ in order to get

$$
\left|R_{3,1,1, i}^{n}\right| \leq \operatorname{ch}_{\mathscr{M}}^{A}\left\|\oiint_{i}\left(u_{i}^{n}-\mathscr{P}_{\mathscr{E}}^{(i)}\left(U_{i}^{n}\right)\right)\right\|_{L^{2}(\Omega)}\left\|\varrho^{n} u_{i}^{n}\right\|_{L^{\frac{6 \gamma}{\gamma+6}(\Omega)}}
$$

If $\gamma \geq 3$, then $\frac{6 \gamma}{\gamma+6} \geq 2$ and we take $p=2, q=2$ in order to obtain

$$
\left|R_{3,1,1, i}^{n}\right| \leq \operatorname{ch}_{\mathscr{M}}^{A}\left\|\oiint_{i}\left(u_{i}^{n}-\mathscr{P}_{\mathscr{E}}^{(i)}\left(U_{i}^{n}\right)\right)\right\|_{L^{2}(\Omega)}\left\|\varrho^{n} u_{i}^{n}\right\|_{L^{\frac{6 \gamma}{\gamma+6}(\Omega)}},
$$

where the constant $c$ depends on $\|\nabla \boldsymbol{U}\|_{L^{\infty}((0, T) \times \Omega)^{3}}$ and on $\eta_{\mathscr{M}}$. Finally from the estimates (3.55), (4.9) and (4.13) we deduce that

$$
\delta t \sum_{n=1}^{N} \sum_{i=1}^{3}\left|R_{3,1,1, i}^{n}\right| \leq \operatorname{ch}_{\mathscr{M}}^{A},
$$

where $c$ depends on $\|\nabla \boldsymbol{U}\|_{L^{\infty}((0, T) \times \Omega)^{3}}, \mathscr{E}_{0, \mathscr{M}}$ and on $\eta_{\mathscr{M}}$. Let us now estimate the remainder $R_{3,1,2, i}^{n}$. Let $K \in \mathscr{M}$ and let us consider $\sigma \in \mathscr{E}^{(i)}(K)$. Without loss of generality we assume that $\sigma=K \mid L \in \mathscr{E}_{\text {int }}^{(i)}$. Let $\epsilon \in \tilde{\mathscr{E}}\left(D_{\sigma}\right)$ such that $\epsilon \neq \epsilon_{K}$ and $\epsilon \cap K \subset \sigma^{\prime} \in \mathscr{E}^{(j)}$ for $j \neq i$ that is $\epsilon \in \tilde{\mathcal{N}}_{\sigma^{\prime}, \sigma}$. Since the primal fluxes vanish on external faces we can assume that $\epsilon \in \tilde{\mathscr{E}}_{\text {int }}^{(i)}$ saying $\epsilon=\sigma \mid \sigma^{\prime \prime}$, where $\sigma^{\prime \prime} \in \tilde{\mathscr{E}}^{(i)}$ that is $\sigma^{\prime \prime} \in \mathcal{N}_{\sigma^{\prime}, \sigma}$. Let $\tilde{K} \in \mathscr{M}$ such that $\sigma^{\prime}=K \mid \tilde{K}$. We define $\sigma^{\prime \prime \prime} \in \mathscr{E}^{(i)}$ such that $\tilde{K}=\left[\sigma^{\prime \prime \prime} \sigma^{\prime \prime}\right]$ and $\sigma^{\prime \prime \prime \prime} \in \mathscr{E}^{(i)}$ such that $K=\left[\sigma^{\prime \prime \prime \prime} \sigma\right]$. Finally let $\tilde{L}$ be the primal cell such that $\sigma^{\prime \prime}=\tilde{K} \mid \tilde{L}$. We summarize the above notations in the figure 10 .

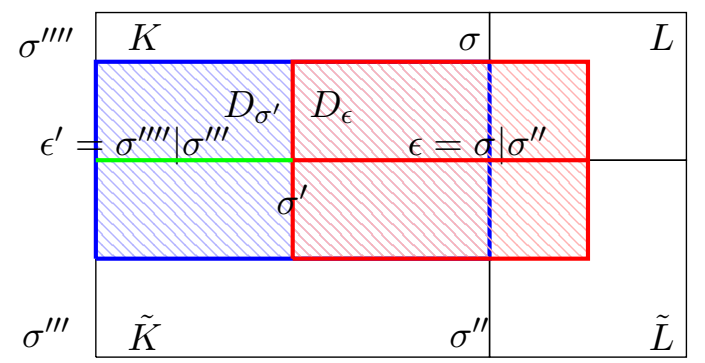

Figure 10: Decomposition of the dual grid

In accordance with the defintion of $u_{\epsilon}^{n}$ and $U_{\epsilon}^{n}$ we can write

$$
\begin{aligned}
\mid \frac{F_{\sigma^{\prime}, K}^{n}}{2}\left(u_{\epsilon}^{n}-U_{\epsilon}^{n}-\right. & \left.\left(\mathscr{R}_{\mathscr{M}}^{(i)}\left(u_{i}^{n}-\mathscr{P}_{\mathscr{E}}^{(i)} U_{i}^{n}\right)\right)_{K}\right) \mid \\
\leq & c\left|\sigma^{\prime}\right| \varrho_{D_{\sigma^{\prime}}}^{n}\left|u_{\sigma^{\prime}}^{n}\right|\left(\frac{|\tilde{K}|}{\left|\sigma^{\prime \prime}\right|}\left|\oiint_{i}\left(u_{i}-\mathscr{P}_{\mathscr{E}}^{(i)} U_{i}^{n}\right)\right|_{\tilde{K}}+d_{\epsilon^{\prime}}\left|\oiint_{j}\left(u_{i}-\mathscr{P}_{\mathscr{E}}^{(i)} U_{i}^{n}\right)\right|_{D_{\epsilon^{\prime}}}\right) \\
\leq & c h_{\sigma^{\prime}}^{-\frac{1}{2}}\left|\sigma^{\prime}\right| \varrho_{D_{\sigma^{\prime}}}^{n}\left|u_{\sigma^{\prime}}^{n}\right|\left(\left\|\partial_{i}\left(u_{i}^{n}-\mathscr{P}_{\mathscr{E}}^{(i)} U_{i}^{n}\right)\right\|_{L^{2}\left(D_{\sigma^{\prime}}\right)}+\left\|\partial_{j}\left(u_{i}^{n}-\mathscr{P}_{\mathscr{E}}^{(i)} U_{i}^{n}\right)\right\|_{L^{2}\left(D_{\sigma^{\prime}}\right)}\right) \\
\leq & \operatorname{ch}_{\mathscr{M}^{A}}^{A} h_{\mathscr{M}}^{-1}\left\|\varrho^{n} u_{j}^{n}\right\|_{L^{\frac{6 \gamma}{\gamma+6}}\left(D_{\sigma^{\prime}}\right)}\left(\left\|\partial_{i}\left(u_{i}^{n}-\mathscr{P}_{\mathscr{E}}^{(i)} U_{i}^{n}\right)\right\|_{L^{2}\left(D_{\sigma^{\prime}}\right)}+\left\|\partial_{j}\left(u_{i}^{n}-\mathscr{P}_{\mathscr{E}}^{(i)} U_{i}^{n}\right)\right\|_{L^{2}\left(D_{\sigma^{\prime}}\right)}\right)
\end{aligned}
$$

We deduce from the previous computation that

$$
\left|R_{3,1,2, i}^{n}\right| \leq c h_{\mathscr{M}}^{A} \sum_{j \neq i} \sum_{\sigma^{\prime} \in \mathscr{E}(j)}\left\|\varrho^{n} u_{j}^{n}\right\|_{L^{\frac{6 \gamma}{\gamma+6}\left(D_{\sigma^{\prime}}\right)}}\left(\left\|\partial_{i}\left(u_{i}^{n}-\mathscr{P}_{\mathscr{E}}^{(i)} U_{i}^{n}\right)\right\|_{L^{2}\left(D_{\sigma^{\prime}}\right)}+\left\|\partial_{j}\left(u_{i}^{n}-\mathscr{P}_{\mathscr{E}}^{(i)} U_{i}^{n}\right)\right\|_{L^{2}\left(D_{\sigma^{\prime}}\right)}\right),
$$

where the constant $c$ depends on $\eta_{\mathscr{M}}$. Finally from the estimates (3.55), (4.9) and (4.13) we deduce, by the similar argument as for the term $R_{3,1,1, i}$, that

$$
\delta t \sum_{n=1}^{N} \sum_{i=1}^{3}\left|R_{3,1,2, i}^{n}\right| \leq c h_{\mathscr{M}}^{A},
$$


where $c$ depends on $\|\nabla \boldsymbol{U}\|_{L^{\infty}((0, T) \times \Omega)^{3}}, \mathscr{E}_{0, \mathscr{M}}$ and on $\eta_{\mathscr{M}}$. Consequently we have

$$
\delta t \sum_{n=1}^{N} \sum_{i=1}^{3}\left(\left|R_{3,1,1, i}^{n}\right|+\left|R_{3,1,2, i}^{n}\right|\right) \leq \operatorname{ch}_{\mathscr{M}}^{A} .
$$

Evidently, for each face $\sigma=K \mid L \in \mathscr{E}_{\text {int }}, u_{\sigma, K}^{n}+u_{\sigma, L}^{n}=0$; whence,

$$
\begin{aligned}
T_{3,1}=\delta t \sum_{n=1}^{m} \sum_{i=1}^{3} \sum_{K \in \mathscr{M}} \sum_{j=1}^{3} \sum_{\sigma \in \mathscr{E}(j)}|\sigma| \varrho_{\sigma}^{n, \mathrm{up}} & u_{\sigma}^{n} \boldsymbol{e}^{(j)} \cdot \boldsymbol{n}_{\sigma, K} \\
& \times\left(\mathscr{R}_{\mathscr{E}}^{(i, j)}\left(u_{i}^{n}-\mathscr{P}_{\mathscr{C}}^{(i)} U_{i}^{n}\right)\right)_{\sigma}\left(\left(\mathscr{R}_{\mathscr{M}}^{(i)} \mathscr{P}_{\mathscr{E}}^{(i)} U_{i}^{n}\right)_{K}-\left(\mathscr{P}_{\mathscr{E}}^{(j)} U_{i}^{n}\right)_{\sigma}\right)
\end{aligned}
$$

Consequently

$$
\begin{aligned}
T_{3,1}=\delta t \sum_{n=1}^{m} \sum_{i=1}^{3} \sum_{K \in \mathscr{M}} \sum_{j=1}^{3} \sum_{\sigma \in \mathscr{E}(j)} \sum_{K)}|\sigma| \varrho_{\sigma}^{n, \text { up }}\left(\mathscr{P}_{\mathscr{E}}^{(j)} U_{j}^{n}\right)_{\sigma} \boldsymbol{e}^{(j)} \cdot \boldsymbol{n}_{\sigma, K} \\
\quad \times\left(\mathscr{R}_{\mathscr{E}}^{(i, j)}\left(u_{i}^{n}-\mathscr{P}_{\mathscr{E}}^{(i)} U_{i}^{n}\right)\right)_{\sigma}\left(\left(\mathscr{R}_{\mathscr{M}}^{(i)} \mathscr{P}_{\mathscr{E}}^{(i)} U_{i}^{n}\right)_{K}-\left(\mathscr{P}_{\mathscr{E}}^{(j)} U_{i}^{n}\right)_{\sigma}\right)+R_{3,2},
\end{aligned}
$$

where $R_{3,2}=\delta t \sum_{n=1}^{m} \sum_{i=1}^{3} \sum_{K \in \mathscr{M}} \sum_{j=1}^{3} \sum_{\sigma \in \mathscr{E}^{(j)}(K) \cap \mathscr{E}_{\text {int }}} R_{3,2, i, K, j, \sigma}^{n}$,

$$
R_{3,2, i, K, j, \sigma}^{n}=|\sigma| \varrho_{\sigma}^{n, \text { up }}\left(u_{\sigma}^{n}-\left(\mathscr{P}_{\mathscr{E}}^{(j)} U_{j}^{n}\right)_{\sigma}\right) \boldsymbol{e}^{(j)} \cdot \boldsymbol{n}_{\sigma, K}\left(\mathscr{R}_{\mathscr{E}}^{(i, j)}\left(u_{i}^{n}-\mathscr{P}_{\mathscr{E}}^{(i)} U_{i}^{n}\right)\right)_{\sigma}\left(\left(\mathscr{R}_{\mathscr{M}}^{(i)} \mathscr{P}_{\mathscr{E}}^{(i)} U_{i}^{n}\right)_{K}-\left(\mathscr{P}_{\mathscr{E}}^{(j)} U_{i}^{n}\right)_{\sigma}\right)
$$

Using (3.49) we infer that for any $(i, j) \in\{1,2,3\}^{2}, K \in \mathscr{M}, \sigma \in \mathscr{E}^{(j)}(K) \cap \mathscr{E}_{\text {int }}$,

$$
\left|\left(\mathscr{R}_{\mathscr{M}}^{(i)} \mathscr{P}_{\mathscr{E}}^{(i)} U_{i}^{n}\right)_{K}-\left(\mathscr{P}_{\mathscr{E}}^{(j)} U_{i}^{n}\right)_{\sigma}\right| \leq c h_{\sigma}
$$

where $c$ depends on $\|\nabla \boldsymbol{U}\|_{L^{\infty}((0, T) \times \Omega)^{3 \times 3}}$ and on $\eta_{\mathscr{M}}$. Consequently we can estimate the general term of $R_{3,2}$ as follows

$$
\begin{array}{r}
\left|R_{3,2, i, K, j, \sigma}^{n}\right|=|| \sigma\left|\varrho_{\sigma}^{n, \mathrm{up}}\left(u_{\sigma}^{n}-\left(\mathscr{P}_{\mathscr{E}}^{(j)} U_{j}^{n}\right)_{\sigma}\right) \boldsymbol{e}^{(j)} \cdot \boldsymbol{n}_{\sigma, K}\left(\mathscr{R}_{\mathscr{E}}^{(i, j)}\left(u_{i}^{n}-\mathscr{P}_{\mathscr{E}}^{(i)} U_{i}^{n}\right)\right)_{\sigma}\left(\left(\mathscr{R}_{\mathscr{M}}^{(i)} \mathscr{P}_{\mathscr{E}}^{(i)} U_{i}^{n}\right)_{K}-\left(\mathscr{P}_{\mathscr{E}}^{(j)} U_{i}^{n}\right)_{\sigma}\right)\right| \\
\leq c\left|D_{\sigma}\right|\left|\varrho_{\sigma}^{n, \mathrm{up}}\right|\left|\left(u_{\sigma}^{n}-\left(\mathscr{P}_{\mathscr{E}}^{(j)} U_{j}^{n}\right)_{\sigma}\right)\right|\left|\left(\mathscr{R}_{\mathscr{E}}^{(i, j)}\left(u_{i}^{n}-\mathscr{P}_{\mathscr{E}}^{(i)} U_{i}^{n}\right)\right)_{\sigma}\right| \\
\left.\leq c\left|D_{\sigma}\right|\left(\varrho_{K}^{n}+\varrho_{L}^{n}\right)\left|\left(u_{\sigma}^{n}-\left(\mathscr{P}_{\mathscr{E}}^{(j)} U_{j}^{n}\right)_{\sigma}\right)\right| \mid\left(\mathscr{R}_{\mathscr{E}}^{(i, j)}\left(u_{i}^{n}-\mathscr{P}_{\mathscr{E}}^{(i)} U_{i}^{n}\right)\right)_{\sigma}\right) \mid \\
\leq c \int_{D_{\sigma}} \varrho^{n}\left|u_{j}^{n}-\mathscr{P}_{\mathscr{E}}^{(j)}\left(U_{j}^{n}\right)\right|\left|\mathscr{R}_{\mathscr{E}}^{(i, j)}\left(u_{i}^{n}-\mathscr{P}_{\mathscr{E}}^{(i)}\left(U_{i}^{n}\right)\right)\right| \mathrm{d} \boldsymbol{x},
\end{array}
$$

where $\sigma=K \mid L \in \mathscr{E}^{(j)}(K) \cap \mathscr{E}_{\text {int }}$. Consequently, using Lemma 3.2, formula (3.65), Hölder's inequality and then Young's inequality, we infer that

$$
\begin{aligned}
&\left|R_{3,2,1}\right| \leq c \delta t \sum_{n=1}^{m} \sum_{i=1}^{3} \sum_{j=1}^{3} \int_{\Omega} \varrho^{n}\left|u_{j}^{n}-\mathscr{P}_{\mathscr{E}}^{(j)}\left(U_{j}^{n}\right) \| \mathscr{R}_{\mathscr{E}}^{(i, j)}\left(u_{i}^{n}-\mathscr{P}_{\mathscr{E}}^{(i)}\left(U_{i}^{n}\right)\right)\right| \mathrm{d} \boldsymbol{x} \\
& \leq c \delta t \sum_{n=1}^{m} \sum_{i=1}^{3} \sum_{j=1}^{3}\left\|\varrho^{n}\right\|_{L^{3 / 2}(\Omega)}^{1 / 2}\left(\mathfrak{E}\left(\varrho^{n}, \boldsymbol{u}^{n} \mid r_{\mathscr{M}}^{n}, \boldsymbol{U}_{\mathscr{E}}^{n}\right)\right)^{1 / 2}\left\|\mathscr{R}_{\mathscr{E}}^{(i, j)}\left(u_{i}^{n}-\mathscr{P}_{\mathscr{E}}^{(i)}\left(U_{i}^{n}\right)\right)\right\|_{L^{6}(\Omega)} \\
& \leq \delta t \sum_{n=1}^{m} \sum_{i=1}^{3} \sum_{j=1}^{3}\left\|\varrho^{n}\right\|_{L^{3 / 2}(\Omega)}^{1 / 2}\left(\mathfrak{E}\left(\varrho^{n}, \boldsymbol{u}^{n} \mid r_{\mathscr{M}}^{n}, \boldsymbol{U}_{\mathscr{E}}^{n}\right)\right)^{1 / 2}\left\|u_{i}^{n}-\mathscr{P}_{\mathscr{E}}^{(i)}\left(U_{i}^{n}\right)\right\|_{1, \mathscr{E}(i)}, 0 \\
& \leq \frac{c}{\delta} \delta t \sum_{n=1}^{m} \mathfrak{E}\left(\varrho^{n}, \boldsymbol{u}^{n} \mid r_{\mathscr{M}}^{n}, \boldsymbol{U}_{\mathscr{E}}^{n}\right)+\delta \delta t \sum_{n=1}^{m}\left\|\boldsymbol{u}^{n}-\boldsymbol{U}_{\mathscr{E}}^{n}\right\|_{1, \mathscr{E}, 0}^{2}
\end{aligned}
$$

where $c$ depends on $\|\nabla \boldsymbol{U}\|_{L^{\infty}((0, T) \times \Omega)}, \mathscr{E}_{0, \mathscr{M}}$ and on $\eta_{\mathscr{M}}$. 
Step 3: Term $T_{5}$. Using the Taylor formula since $p \in C^{2}\left(\mathbb{R}_{+}^{\star}\right)$ we get

$$
\mathscr{H}^{\prime}\left(r_{K}^{n}\right)-\mathscr{H}^{\prime}\left(r_{K}^{n-1}\right)=\mathscr{H}^{\prime \prime}\left(r_{K}^{n}\right)\left(r_{K}^{n}-r_{K}^{n-1}\right)-\frac{1}{2} \mathscr{H}^{\prime \prime \prime}\left(\bar{r}_{K}^{n}\right)\left(r_{K}^{n}-r_{K}^{n-1}\right)^{2},
$$

where $\bar{r}_{K}^{n} \in\left[\min \left(r_{K}^{n-1}, r_{K}^{n}\right), \max \left(r_{K}^{n-1}, r_{K}^{n}\right)\right]$. Consequently $T_{5}=T_{5,1}+R_{5,1}$ with

$$
T_{5,1}=\delta t \sum_{n=1}^{m} \int_{\Omega}\left(r_{\mathscr{M}}^{n}-\varrho^{n}\right) \frac{p^{\prime}\left(r_{\mathscr{M}}^{n}\right)}{r_{\mathscr{M}}^{n}} \frac{r_{\mathscr{M}}^{n}-r_{\mathscr{M}}^{n-1}}{\delta t} \mathrm{~d} \boldsymbol{x}
$$

and

$$
R_{5,1}=\delta t \sum_{n=1}^{m} \sum_{K \in \mathscr{M}} R_{5,1}^{n, K}, R_{5,1}^{n, K}=\frac{1}{2}|K| \mathscr{H}^{\prime \prime \prime}\left(\bar{r}_{K}^{n}\right) \frac{\left(r_{K}^{n}-r_{K}^{n-1}\right)^{2}}{\delta t}\left(\varrho_{K}^{n}-r_{K}^{n}\right) .
$$

By the first order Taylor formula applied to function $t \mapsto r(t, x)$ on the interval $\left(t^{n-1}, t^{n}\right)$, thanks to the relation (2.3), to the mass conservation (3.16) and (4.12) we have

$$
\left|R_{5,1}\right| \leq c \delta t
$$

where $c$ depends on $\left\|\partial_{t} r\right\|_{L^{\infty}\left(Q_{T}\right)}$ and on $\mathscr{E}_{0, \mathscr{M}}$.

Let us now decompose $T_{5,1}$ as follows: $T_{5,1}=T_{5,2}+R_{5,2}$ with

$$
T_{5,2}=\delta t \sum_{n=1}^{m} \sum_{K \in \mathscr{M}} \int_{K}\left(r_{\mathscr{M}}^{n}-\varrho^{n}\right) \frac{p^{\prime}\left(r_{\mathscr{M}}^{n}\right)}{r_{\mathscr{M}}^{n}}\left[\partial_{t} r\right]^{n} \mathrm{~d} x,
$$

and

$$
R_{5,2}=\delta t \sum_{n=1}^{m} \int_{\Omega}\left(r_{\mathscr{M}}^{n}-\varrho^{n}\right) \frac{p^{\prime}\left(r_{\mathscr{M}}^{n}\right)}{r_{\mathscr{M}}^{n}}\left(\frac{r_{\mathscr{M}}^{n}-r_{\mathscr{M}}^{n-1}}{\delta t}-\left[\partial_{t} r\right]^{n}\right) \mathrm{d} \boldsymbol{x}
$$

Using twice the Taylor formula, the Fubini Theorem and Hölder's inequality, we can obtain, as in [24],

$$
\left|R_{5,2}\right| \leq c \delta t
$$

where $c$ depends on $\underline{r}, \bar{r},\left\|\partial_{t}^{2} r\right\|_{L^{1}\left(0, T ; L \gamma^{\prime}(\Omega)\right)}$ and on $\mathscr{E}_{0, \mathscr{M}}$.

Step 4: Term $T_{6}$. Using the local conservation of the flux through primal faces, we may write

$$
\begin{aligned}
& T_{6}=T_{6,1}+R_{6,1}, \quad R_{6,1}=\delta t \sum_{n=1}^{m} \sum_{K \in \mathscr{M}} \sum_{\sigma \in \mathscr{E}(K)} R_{6,1}^{n, \sigma, K}, \text { with } \\
& T_{6,1}=\delta t \sum_{n=1}^{m} \sum_{\sigma \in \mathscr{E}(K) \cap \mathscr{E}_{\mathrm{int}}}|\sigma| \varrho_{K}^{n}\left(\mathscr{H}^{\prime}\left(r_{K}^{n-1}\right)-\mathscr{H}^{\prime}\left(r_{\sigma}^{n-1}\right)\right) u_{\sigma, K}^{n}, \text { and } \\
& R_{6,1}^{n, \sigma, K}=|\sigma|\left(\varrho_{\sigma}^{n, \text { up }}-\varrho_{K}^{n}\right)\left(\mathscr{H}^{\prime}\left(r_{K}^{n-1}\right)-\mathscr{H}^{\prime}\left(r_{\sigma}^{n-1}\right)\right) u_{\sigma, K}^{n}, \sigma \in \mathscr{E}(K) \cap \mathscr{E}_{\mathrm{int}} .
\end{aligned}
$$

Motivated by (4.16), we find for $\sigma=K \mid L \in \mathscr{E}_{\text {int }}$

$$
\begin{aligned}
& \left|R_{6,1}^{n, \sigma, K}\right| \leq c \sqrt{h_{\mathscr{M}}}|\sigma| \\
& \times\left(\frac{\left|\varrho_{\sigma}^{n, \text { up }}-\varrho_{K}^{n}\right|}{\max \left(\varrho_{K}^{n}, \varrho_{L}^{n}\right)^{(2-\gamma)^{+} / 2}} \sqrt{\mid u_{\sigma, K}^{n}} \mid 1_{\varrho_{\sigma}^{n} \geq 1} \sqrt{h_{K}}\left(\varrho_{K}^{n}+\varrho_{L}^{n}\right)^{(2-\gamma)^{+} / 2} \sqrt{\left|u_{\sigma, K}^{n}\right|}\right. \\
& \left.+\left|\varrho_{\sigma}^{n, \text { up }}-\varrho_{K}^{n}\right| \sqrt{\left|u_{\sigma, K}^{n}\right|} 1_{\varrho_{\sigma}^{n}<1} \sqrt{h_{K}} \sqrt{\left|u_{\sigma, K}^{n}\right|}\right),
\end{aligned}
$$

where $c$ depends on $\underline{r},\|\nabla r\|_{L^{\infty}((0, T) \times \Omega)^{3}}$ and where the numbers $\bar{\varrho}_{\sigma}^{n}$ are defined in Theorem 4.1. Here we have used the first order Taylor formula applied to function $\mathscr{H}^{\prime}$ between endpoints $r_{K}^{n-1}, r_{\sigma}^{n-1}$. Conse- 
quently the Hölder and Young inequalities give

$$
\begin{aligned}
\left|R_{6,1}\right| \leq & c \sqrt{h_{\mathscr{M}}} \delta t \sum_{n=1}^{m}\left[\left(\sum_{K \in \mathscr{M}} \sum_{\sigma=K \mid L \in \mathscr{C}(K)}|\sigma| \frac{\left(\varrho_{\sigma}^{n, \text { up }}-\varrho_{K}^{n}\right)^{2}}{\max \left(\varrho_{K}^{n}, \varrho_{L}^{n}\right)^{(2-\gamma)^{+}}}\left|u_{\sigma, K}^{n}\right| 1_{\left\{\varrho_{\sigma}^{n} \geq 1\right\}}\right)^{1 / 2}\right. \\
& \times\left(\sum_{K \in \mathscr{M}} \sum_{\sigma \in \mathscr{E}(K)}|\sigma| h_{\sigma}\left(\varrho_{K}^{n}\right)^{(2-\gamma)^{+}}\left|u_{\sigma, K}^{n}\right|\right)^{1 / 2} \\
& \left.+\left(\sum_{K \in \mathscr{M}} \sum_{\sigma=K \mid L \in \mathscr{E}(K)}|\sigma|\left(\varrho_{\sigma}^{n, \text { up }}-\varrho_{K}^{n}\right)^{2}\left|u_{\sigma, K}^{n}\right| 1_{\left\{\varrho_{\sigma}^{n}<1\right\}}\right)^{1 / 2}\left(\sum_{K \in \mathscr{M}} \sum_{\sigma \in \mathscr{E}(K)}|\sigma| h_{\sigma}\left|u_{\sigma, K}^{n}\right|\right)^{1 / 2}\right] \\
\leq & c \sqrt{h_{\mathscr{M}}} \delta t \sum_{n=1}^{m}\left[\left(\sum_{K \in \mathscr{M}} \sum_{\sigma=K \mid L \in \mathscr{C}(K)}|\sigma| \frac{\left(\varrho_{\sigma}^{n, \text { up }}-\varrho_{K}^{n}\right)^{2}}{\max \left(\varrho_{K}^{n}, \varrho_{L}^{n}\right)^{(2-\gamma)^{+}}}\left|u_{\sigma, K}^{n}\right| 1_{\varrho_{\sigma}^{n} \geq 1}\right.\right. \\
& +\left(\sum_{K \in \mathscr{M}}|K|\left(\varrho_{K}^{n}\right)^{6(2-\gamma)^{+} / 5}\right)^{5 / 6}\left(\sum_{\sigma \in \mathscr{E}}|\sigma| h_{\sigma}\left|u_{\sigma, K}^{n}\right|^{6}\right)^{1 / 6} \\
& \left.+\sum_{K \in \mathscr{M}} \sum_{\sigma=K \mid L \in \mathscr{E}(K)}|\sigma|\left(\varrho_{\sigma}^{n, \text { up }}-\varrho_{K}^{n}\right)^{2}\left|u_{\sigma, K}^{n}\right| 1_{\left\{\varrho_{K}^{n}<1\right\}}+|\Omega|^{5 / 6}\left(\sum_{\sigma \in \mathscr{E}}|\sigma| h_{\sigma}\left|u_{\sigma, K}^{n}\right|^{6}\right)^{1 / 6}\right] \\
\leq & c \sqrt{h_{\mathscr{M}}}
\end{aligned}
$$

provided $\gamma \geq 12 / 11$, where $c$ depends on $\underline{r}, \bar{r},\|\nabla r\|_{L^{\infty}((0, T) \times \Omega)^{3}}, \mathscr{E}_{0, \mathcal{M}}$. Here we have used estimate (4.16) and estimates (4.10), (4.12) of Corollary 4.1.

Let us now decompose the term $T_{6,1}$ as $T_{6,1}=T_{6,2}+R_{6,2}$ with

$$
T_{6,2}=\delta t \sum_{n=1}^{m} \sum_{K \in \mathscr{M}} \sum_{\sigma \in \mathscr{E}(K) \cap \mathscr{E}_{\text {int }}}|\sigma| \varrho_{K}^{n} \mathscr{H}^{\prime \prime}\left(r_{K}^{n-1}\right)\left(r_{K}^{n-1}-r_{\sigma}^{n-1}\right) u_{\sigma, K}^{n},
$$

where

$$
R_{6,2}=\delta t \sum_{n=1}^{m} \sum_{K \in \mathscr{M}} \sum_{\sigma \in \mathscr{E}(K) \cap \mathscr{E}_{\mathrm{int}}} R_{6,2}^{n, \sigma, K}
$$

and

$$
R_{6,2}^{n, \sigma, K}=|\sigma| \varrho_{K}^{n}\left(\mathscr{H}^{\prime}\left(r_{K}^{n-1}\right)-\mathscr{H}^{\prime}\left(r_{\sigma}^{n-1}\right)-\mathscr{H}^{\prime \prime}\left(r_{K}^{n-1}\right)\left(r_{K}^{n-1}-r_{\sigma}^{n-1}\right)\right) u_{\sigma, K}^{n}
$$

Therefore, by virtue of the second order Taylor formula applied to function $\mathscr{H}^{\prime}$, Hölder's inequality and (3.49), (3.50), (4.9), (4.13) in Corollary 4.1, we obtain,

$$
\left|R_{6,2}\right| \leq \mathrm{ch}_{\mathscr{M}}
$$

where $c$ depends on $\underline{r}, \bar{r},\|\nabla r\|_{L^{\infty}((0, T) \times \Omega)^{3}}, \mathscr{E}_{0, \mathscr{M}}$ and on $\eta_{\mathscr{M}}$.

Let us now deal with the term $T_{6,2}$. First of all, let us remark that $\int_{K} \nabla r^{n-1} \mathrm{~d} \boldsymbol{x}=\sum_{\sigma \in \mathscr{E}(K)}|\sigma|\left(r_{\sigma}^{n-1}-\right.$ $\left.r_{K}^{n-1}\right) \boldsymbol{n}_{\sigma, K}$. Therefore we may write

$$
T_{6,2}=T_{6,3}+R_{6,3}
$$

with

$$
T_{6,3}=-\delta t \sum_{n=1}^{m} \int_{\Omega} \varrho^{n} \mathscr{H}^{\prime \prime}\left(r_{\mathscr{M}}^{n-1}\right) \mathscr{R}_{\mathscr{M}}\left(\boldsymbol{u}^{n}\right) \cdot \nabla r^{n-1} \mathrm{~d} \boldsymbol{x},
$$

where $\mathscr{R}_{\mathscr{M}}$ is defined in (3.64) and where the remainder $R_{6,3}$ is given by

$$
R_{6,3}=\delta t \sum_{n=1}^{m} \sum_{K \in \mathscr{M}} \sum_{j=1}^{3} \sum_{\sigma \in \mathscr{C}(j)}|\sigma| \varrho_{K}^{n} \mathscr{H}^{\prime \prime}\left(r_{K}^{n-1}\right)\left(r_{K}^{n-1}-r_{\sigma}^{n-1}\right)\left(u_{\sigma}^{n}-\left(\mathscr{R}_{\mathscr{M}}^{(j)} u_{j}^{n}\right)_{K}\right) \boldsymbol{e}^{(j)} \cdot \boldsymbol{n}_{\sigma, K}
$$


By virtue of (3.49) and (3.50)

$$
\left|R_{6,3}\right| \leq \operatorname{ch}{ }_{\mathscr{M}} \delta t \sum_{n=1}^{m} \sum_{K \in \mathscr{M}} \sum_{j=1}^{3} \sum_{\sigma \in \mathscr{C}(j)(K)}\left|D_{\sigma}\right| \varrho_{K}^{n}\left|\left(\oiint_{j} u_{j}^{n}\right)_{K}\right| \leq \operatorname{ch}{ }_{\mathscr{M}} \delta t \sum_{n=1}^{m} \sum_{j=1}^{3} \sum_{K \in \mathscr{M}}|K| \varrho_{K}^{n} \cdot\left|\left(\oiint_{j} u_{j}^{n}\right)_{K}\right|
$$

By virtue of the Hölder's inequality, (4.9), (4.12) in Corollary 4.1 we have for $\gamma \geq 2$

$$
\left|R_{6,3}\right| \leq \operatorname{ch}_{\mathscr{M}}
$$

By virtue of the Hölder's inequality, (4.9), (4.12) in Corollary 4.1 we have for $\frac{3}{2} \leq \gamma \leq 2$

$$
\begin{aligned}
& \left|R_{6,3}\right| \leq \operatorname{ch}_{\mathscr{M}^{\frac{5 \gamma-6}{2 \gamma}}} \delta t \sum_{n=1}^{m} \sum_{j=1}^{3} \sum_{K \in \mathscr{M}}\left\|\varrho^{n}\right\|_{L^{\gamma}(K)}\left\|{\partial_{j}}_{j} u_{j}^{n}\right\|_{L^{2}(K)} \\
& \leq \operatorname{ch}_{\mathscr{M}^{\frac{5 \gamma-6}{2 \gamma}}} \delta t \sum_{n=1}^{m} \sum_{j=1}^{3}\left\|{\varpi_{j}}_{j} u_{j}^{n}\right\|_{L^{2}(\Omega)}\left(\sum_{K \in \mathscr{M}}\left\|\varrho^{n}\right\|_{L^{\gamma}(K)}^{2}\right)^{1 / 2} \\
& \leq \operatorname{ch}_{\mathscr{M}^{\frac{5 \gamma-6}{2 \gamma}}}^{2 \gamma} \sum_{n=1}^{m} \sum_{j=1}^{3}\left\|\oiint_{j} u_{j}^{n}\right\|_{L^{2}(\Omega)}\left\|\varrho^{n}\right\|_{L^{\gamma}(\Omega)} \leq \operatorname{ch}_{\mathscr{M}^{\frac{5 \gamma-6}{2 \gamma}}} .
\end{aligned}
$$

Consequently

$$
\left|R_{6,3}\right| \leq \operatorname{ch}_{\mathscr{M}}^{A}
$$

where $c$ depends on $\underline{r}, \bar{r},\|\nabla r\|_{L^{\infty}\left(Q_{T} ; \mathbb{R}^{3}\right)}, \mathscr{E}_{0, \mathscr{M}}$ and on $\eta_{\mathscr{M}}$ and where $A$ is defined in (3.79).

Finally we write $T_{6,3}=T_{6,4}+R_{6,4}$, with

$$
\begin{aligned}
T_{6,4} & =-\delta t \sum_{n=1}^{m} \int_{\Omega} \varrho^{n} \frac{p^{\prime}\left(r_{\mathscr{M}}^{n}\right)}{r_{\mathscr{M}}^{n}} \mathscr{R}_{\mathscr{M}}\left(\boldsymbol{u}^{n}\right) \cdot \nabla r^{n} \mathrm{~d} \boldsymbol{x}, \\
R_{6,4} & =\delta t \sum_{n=1}^{m} \int_{\Omega} \varrho^{n}\left(\mathscr{H}^{\prime \prime}\left(r_{\mathscr{M}}^{n}\right) \nabla r^{n}-\mathscr{H}^{\prime \prime}\left(r_{\mathscr{M}}^{n-1}\right) \nabla r^{n-1}\right) \cdot \mathscr{R}_{\mathscr{M}}\left(\boldsymbol{u}^{n}\right) \mathrm{d} \boldsymbol{x},
\end{aligned}
$$

where by the same token as above the remainder $R_{6,4}$ satisfies

$$
\left|R_{6,4}\right| \leq c \delta t
$$

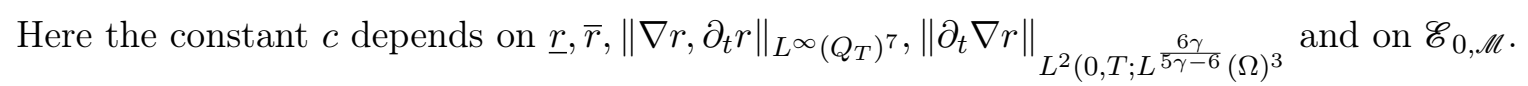

We are now in position to conclude the proof of Lemma 5.1: we obtain the inequality (5.14) by gathering the principal terms (5.19), (5.23), (5.26), (5.35) and the residual terms estimated in (5.18), (5.20), (5.22), (5.25), (5.27), (5.30), (5.32), (5.34), (5.36) at the right hand side $\sum_{i=1}^{6} T_{i}$ of the discrete relative energy inequality (5.1).

\section{A consistency error}

This section is devoted to the derivation of a discrete identity satisfied by any strong solution. This identity is stated in Lemma 6.1 below. It will be used in combination with the approximate relative energy inequality stated in Lemma 5.1 to deduce the convenient form of the relative energy inequality verified by any function being a strong solution to the compressible Navier-Stokes system. This last step is performed in the next section.

Lemma 6.1 (Consistency error). Let $(\varrho, \boldsymbol{u}) \in Y_{\mathscr{M}, \delta t} \times \boldsymbol{X}_{\mathscr{C}, \delta t}$ be a solution of the discrete problem (3.10). Let $(r, \boldsymbol{U})$ belonging to the class (3.73) be such that $\boldsymbol{U}_{\mid(0, T) \times \partial \Omega}=0$ and satisfies (1.1). Then there exists 
$c$ depending only on parameters (5.11-5.13) such that for any $m=1, \ldots, N$, the following identity holds:

$$
\begin{aligned}
& \delta t \sum_{n=1}^{m}\left(\mu\left[\boldsymbol{U}_{\mathscr{E}}^{n}, \boldsymbol{u}^{n}-\boldsymbol{U}_{\mathscr{E}}^{n}\right]_{1, \mathscr{E}, 0}\right.\left.+(\mu+\lambda) \int_{\Omega} \operatorname{div} \boldsymbol{U}^{n} \operatorname{div} \mathscr{M}\left(\boldsymbol{u}^{n}-\boldsymbol{U}_{\mathscr{E}}^{n}\right) \mathrm{d} \boldsymbol{x}\right) \\
&+\delta t \sum_{n=1}^{m} \int_{\Omega} r_{\mathscr{M}}^{n-1} \frac{\boldsymbol{U}_{\mathscr{E}}^{n}-\boldsymbol{U}_{\mathscr{E}}^{n-1}}{\delta t} \cdot\left(\boldsymbol{u}^{n}-\boldsymbol{U}_{\mathscr{E}}^{n}\right) \mathrm{d} \boldsymbol{x} \\
&+\delta t \sum_{n=1}^{m} \sum_{i=1}^{3} \sum_{K \in \mathscr{M}} \sum_{j=1}^{3} \sum_{\sigma \in \mathscr{E}(j)}\left[|\sigma| r_{\sigma}^{n, \mathrm{up}}\left(\mathscr{P}_{\mathscr{E}}^{(j)} U_{j}^{n}\right)_{\sigma} \boldsymbol{e}^{(j)} \cdot \boldsymbol{n}_{\sigma, K}\left(\mathscr{R}_{\mathscr{E}}^{(i, j)}\left(u_{i}^{n}-\mathscr{P}_{\mathscr{E}}^{(i)} U_{i}^{n}\right)\right)_{\sigma}\right. \\
&\left.\quad \times\left(\left(\mathscr{R}_{\mathscr{E}}^{(i, j)} U_{i}^{n}\right)_{\sigma}-\left(\mathscr{R}_{\mathscr{M}}^{(i)} \mathscr{P}_{\mathscr{E}}^{(i)} U_{i}^{n}\right)_{K}\right)\right] \\
&+\delta t \sum_{n=1}^{m} \int_{\Omega} p\left(r_{\mathscr{M}}^{n}\right) \operatorname{div} \boldsymbol{U}^{n} \mathrm{~d} \boldsymbol{x}+\delta t \sum_{n=1}^{m} \int_{\Omega} p^{\prime}\left(r_{\mathscr{M}}^{n}\right) \mathscr{R}_{\mathscr{M}}\left(\boldsymbol{u}^{n}\right) \cdot \nabla r^{n} \mathrm{~d} \boldsymbol{x}+\mathscr{K}_{\mathscr{M}, \delta t}^{m}=0,
\end{aligned}
$$

where the remainder $\mathscr{K}_{\mathscr{M}, \delta t}^{m}$ satisfies

$$
\left|\mathscr{K}_{\mathscr{M}, \delta t}^{m}\right| \leq c\left(h_{\mathscr{M}}+\delta t\right)
$$

Proof. Since $(r, \boldsymbol{U})$ satisfies (1.1) and belongs to the class (3.73), Equation (1.1b) can be rewritten in the form

$$
r \partial_{t} \boldsymbol{U}+r \boldsymbol{U} \cdot \nabla \boldsymbol{U}+\nabla p(r)=\mu \Delta \boldsymbol{U}+(\mu+\lambda) \nabla \operatorname{div} \boldsymbol{U} \text { in }(0, T) \times \Omega .
$$

We write equation (6.2) at $t=t^{n}$, multiply scalarly by $\boldsymbol{u}^{n}-\boldsymbol{U}_{\mathscr{E}}^{n}$, and integrate over $\Omega$. We get, after summation from $n=1$ to $m, \sum_{i=1}^{5} Q_{i}=0$, where

$$
\begin{aligned}
& Q_{1}=\delta t \sum_{n=1}^{m} \int_{\Omega} r^{n} \partial_{t} \boldsymbol{U}^{n} \cdot\left(\boldsymbol{u}^{n}-\boldsymbol{U}_{\mathscr{E}}^{n}\right) \mathrm{d} \boldsymbol{x}, \\
& Q_{2}=\delta t \sum_{n=1}^{m} \int_{\Omega} r^{n} \boldsymbol{U}^{n} \cdot \nabla \boldsymbol{U}^{n} \cdot\left(\boldsymbol{u}^{n}-\boldsymbol{U}_{\mathscr{E}}^{n}\right) \mathrm{d} \boldsymbol{x}, \\
& Q_{3}=\delta t \sum_{n=1}^{m} \int_{\Omega} \nabla p\left(r^{n}\right) \cdot\left(\boldsymbol{u}^{n}-\boldsymbol{U}_{\mathscr{E}}^{n}\right) \mathrm{d} \boldsymbol{x} \\
& Q_{4}=-\delta t \sum_{n=1}^{m} \int_{\Omega} \mu \Delta \boldsymbol{U}^{n} \cdot\left(\boldsymbol{u}^{n}-\boldsymbol{U}_{\mathscr{E}}^{n}\right) \mathrm{d} \boldsymbol{x}, \\
& Q_{5}=-\delta t \sum_{n=1}^{m} \int_{\Omega}(\mu+\lambda) \nabla \operatorname{div} \boldsymbol{U}^{n} \cdot\left(\boldsymbol{u}^{n}-\boldsymbol{U}_{\mathscr{E}}^{n}\right) \mathrm{d} \boldsymbol{x} .
\end{aligned}
$$

An adaptation of the proof of Lemma 7.1 in [24] gives

$$
\begin{aligned}
Q_{1}+Q_{3}=\delta t \sum_{n=1}^{m} \int_{\Omega} r_{\mathscr{M}}^{n-1} \frac{\boldsymbol{U}_{\mathscr{E}}^{n}-\boldsymbol{U}_{\mathscr{E}}^{n-1}}{\delta t}\left(\boldsymbol{u}^{n}-\boldsymbol{U}_{\mathscr{E}}^{n}\right) \mathrm{d} \boldsymbol{x} & +\delta t \sum_{n=1}^{m} \int_{\Omega} p\left(r_{\mathscr{M}}^{n}\right) \operatorname{div} \boldsymbol{U}^{n} \mathrm{~d} \boldsymbol{x} \\
& +\delta t \sum_{n=1}^{m} \int_{\Omega} p^{\prime}\left(r_{\mathscr{M}}^{n}\right) \mathscr{R}_{\mathscr{M}}\left(\boldsymbol{u}^{n}\right) \cdot \nabla r^{n} \mathrm{~d} \boldsymbol{x}+R_{1},
\end{aligned}
$$

where the remainder $R_{1}$ satisfies

$$
\left|R_{1}\right| \leq c\left(h_{\mathscr{M}}+\delta t\right) .
$$

and where the constant $c$ depends on $\bar{r},|p|_{C^{2}([\underline{r}, \bar{r}])},\|\nabla r\|_{L^{\infty}((0, T) \times \Omega)},\left\|\partial_{t} \boldsymbol{U}_{L^{\infty}((0, T) \times \Omega)},\right\| \nabla \boldsymbol{U} \|_{L^{\infty}((0, T) \times \Omega)}$ and on $\left\|\partial_{t}^{2} \boldsymbol{U}\right\|_{L^{2}\left(0, T ; L^{\frac{6}{5}}(\Omega)\right)},\left\|\partial_{t} \nabla \boldsymbol{U}\right\|_{L^{2}\left(0, T ; L^{\frac{6}{5}}(\Omega)\right)}, \mathscr{E}_{0, \mu}$. 
By virtue of the Stoke's formula we transform the term $Q_{4}$ as follows, using (3.38), (3.40) and $d_{\epsilon}|\epsilon|=\left|D_{\epsilon}\right|$,

$$
\begin{aligned}
& Q_{4}=-\delta t \sum_{n=1}^{m} \int_{\Omega} \mu \Delta \boldsymbol{U}^{n} \cdot\left(\boldsymbol{u}^{n}-\boldsymbol{U}_{\mathscr{E}}^{n}\right) \mathrm{d} \boldsymbol{x}=-\delta t \mu \sum_{n=1}^{m} \sum_{i=1}^{3} \sum_{j=1}^{3} \sum_{\substack{\epsilon \in \tilde{\mathscr{E}}^{(i)} \\
\epsilon \perp \boldsymbol{e}^{(j)}}} \int_{D_{\epsilon}} \frac{\partial^{2}}{\partial_{x_{j}}^{2}} U_{i}^{n}\left(u_{i}^{n}-\left(\mathscr{P}_{\mathscr{E}}^{(i)} U_{i}^{n}\right)\right) \mathrm{d} \boldsymbol{x} \\
& =\delta t \mu \sum_{n=1}^{m} \sum_{i=1}^{3} \sum_{j=1}^{3} \sum_{\substack{\epsilon \in \widetilde{\mathscr{E}}(i) \\
\epsilon \perp \boldsymbol{e}^{(j)}}} d_{\epsilon}|\epsilon|\left(\check{\partial}_{j}\left(u_{i}^{n}-P p_{\mathscr{\mathscr { C }}}^{(i)} U_{i}^{n}\right)\right)_{D_{\epsilon}} \frac{1}{|\epsilon|} \int_{\epsilon} \frac{\partial}{\partial x_{j}} U_{i}^{n} \mathrm{~d} \gamma \\
& =\delta t \mu \sum_{n=1}^{m} \sum_{i=1}^{3} \sum_{j=1}^{3} \sum_{\substack{\epsilon \in \widetilde{\mathscr{E}}(i) \\
\epsilon \perp \boldsymbol{e}^{(j)}}} d_{\epsilon}|\epsilon|\left(\Im_{j}\left(u_{i}^{n}-\mathscr{P}_{\mathscr{E}}^{(i)} U_{i}^{n}\right)\right)_{D_{\epsilon}}\left({\coprod_{j}}_{j}\left(\mathscr{P}_{\mathscr{E}}^{(i)} U_{i}^{n}\right)\right)_{D_{\epsilon}}+R_{\mathscr{M}, \delta t}^{m} \\
& =\delta t \sum_{n=1}^{m} \mu\left[\boldsymbol{U}_{\mathscr{E}}^{n}, \boldsymbol{u}^{n}-\boldsymbol{U}_{\mathscr{E}}^{n}\right]_{1, \mathscr{E}, 0}+R_{4},
\end{aligned}
$$

where the remainder $R_{4}$ is given by

$$
R_{4}=\delta t \mu \sum_{n=1}^{m} \sum_{i=1}^{3} \sum_{j=1}^{3} \sum_{\substack{\epsilon \in \widetilde{\mathscr{E}}(i) \\ \epsilon \perp \boldsymbol{e}^{(j)}}} d_{\epsilon}|\epsilon|\left(\varlimsup_{j}\left(u_{i}^{n}-\mathscr{P}_{\mathscr{E}}^{(i)} U_{i}^{n}\right)\right)_{D_{\epsilon}}\left(\frac{1}{|\epsilon|} \int_{\epsilon} \frac{\partial}{\partial x_{j}} U_{i}^{n} \mathrm{~d} \gamma-\left(\varlimsup_{j}\left(\mathscr{P}_{\mathscr{E}}^{(i)} U_{i}^{n}\right)\right)_{D_{\epsilon}}\right) .
$$

Moreover by virtue of (3.51) and (3.52) in Lemma 3.3 we can write for $\epsilon=\overrightarrow{\sigma \mid \sigma^{\prime}} \in \tilde{\mathscr{E}}_{\text {int }}^{(i)}, \epsilon \perp \boldsymbol{e}^{(j)}$,

$$
\begin{aligned}
\left(\check{\partial}_{j} \mathscr{P}_{\mathscr{E}}^{(i)} U_{i}^{n}\right)_{D_{\epsilon}}-\frac{1}{|\epsilon|} \int_{\epsilon} \frac{\partial}{\partial x_{j}} U_{i}^{n} \mathrm{~d} \gamma=\frac{1}{d_{\epsilon}}\left(U_{i}\left(\boldsymbol{x}_{\sigma^{\prime}}\right)-U_{i}\left(\boldsymbol{x}_{\sigma}\right)\right)-\frac{\partial}{\partial x_{j}} U_{i}^{n}\left(\boldsymbol{x}_{\epsilon}\right)+R_{\epsilon}^{n} & \\
& =\frac{\partial}{\partial x_{j}} U_{i}^{n}\left(\boldsymbol{x}_{\sigma, \sigma^{\prime}}\right)-\frac{\partial}{\partial x_{j}} U_{i}^{n}\left(\boldsymbol{x}_{\epsilon}\right)+R_{\epsilon}^{n},
\end{aligned}
$$

where $\boldsymbol{x}_{\sigma, \sigma^{\prime}} \in \boldsymbol{x}_{\sigma} \boldsymbol{x}_{\sigma^{\prime}}$ and where the remainder $R_{\epsilon}^{n}$ satisfies

$$
\left|R_{\epsilon}^{n}\right| \leq \operatorname{ch}_{\mathscr{M}}
$$

Note that the case $\epsilon \in \tilde{\mathscr{E}}_{\text {ext }}^{(i)}$ can be treated in the same way. Consequently we have inequality

$$
\left|\frac{1}{|\epsilon|} \int_{\epsilon} \frac{\partial}{\partial x_{j}} U_{i}^{n} \mathrm{~d} \gamma-\left(\check{\partial}_{j} \mathscr{P}_{\mathscr{E}}^{(i)} U_{i}^{n}\right)_{D_{\epsilon}}\right| \leq c h_{\mathscr{M}}, \forall(i, j) \in\{1,2,3\}^{2}, \forall \epsilon \in \widetilde{\mathscr{E}}^{(i)}, \epsilon \perp \boldsymbol{e}^{(j)},
$$

where the constant $c$ depends on $\left\|\nabla^{2} \boldsymbol{U}\right\|_{L^{\infty}((0, T) \times \Omega)}$. Therefore

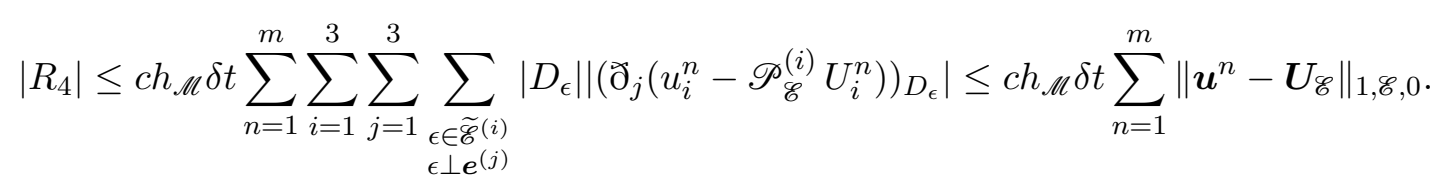

Consequently by virtue of (3.55) and (4.9) we have

$$
Q_{4}=\delta t \sum_{n=1}^{m} \mu\left[\boldsymbol{U}_{\mathscr{E}}^{n}, \boldsymbol{u}^{n}-\boldsymbol{U}_{\mathscr{E}}^{n}\right]_{1, \mathscr{E}, 0}+R_{4},
$$

where the remainder $R_{4}$ satisfies

$$
\left|R_{4}\right| \leq \operatorname{ch}_{\mathscr{M}}
$$

with $c$ dependent on $\|\nabla \boldsymbol{U}\|_{L^{\infty}((0, T) \times \Omega)},\left\|\nabla^{2} \boldsymbol{U}\right\|_{L^{\infty}((0, T) \times \Omega)}, \eta_{\mathscr{M}}$ and $\mathscr{E}_{0, \mathscr{M}}$.

The term $Q_{5}$ can be treated exactly in the same way as $Q_{4}$ in order to obtain

$$
Q_{5}=-\delta t \sum_{n=1}^{m}(\mu+\lambda) \int_{\Omega} \operatorname{div} \boldsymbol{U}^{n} \operatorname{div}_{\mathscr{M}}\left(\boldsymbol{u}^{n}-\boldsymbol{U}_{\mathscr{E}}^{n}\right) \mathrm{d} \boldsymbol{x}+R_{5}
$$


where the remainder $R_{5}$ satisfies

$$
\left|R_{5}\right| \leq \operatorname{ch}_{\mathscr{M}} .
$$

where the constant $c$ depends on $\|\nabla \boldsymbol{U}\|_{L^{\infty}((0, T) \times \Omega)}, \| \nabla^{2} \boldsymbol{U}_{L^{\infty}((0, T) \times \Omega)}, \eta_{\mathscr{M}}$ and on $\mathscr{E}_{0, \mathscr{M}}$.

Let us deal with the term $Q_{2}$. We have

$$
\begin{aligned}
Q_{2}=\delta t \sum_{n=1}^{m} \sum_{i=1}^{3} \int_{\Omega} r^{n} \boldsymbol{U}^{n} \cdot \nabla U_{i}^{n}\left(u_{i}^{n}-\mathscr{P}_{\mathscr{E}}^{(i)}\left(U_{i}^{n}\right)\right) \mathrm{d} \boldsymbol{x} \\
\quad=\delta t \sum_{n=1}^{m} \sum_{i=1}^{3} \sum_{j=1}^{3} \sum_{K \in \mathscr{M}} \int_{K} r_{K}^{n}\left(\mathscr{R}_{\mathscr{M}}^{(j)} \mathscr{P}_{\mathscr{E}}^{(j)} U_{j}^{n}\right)_{K} \partial_{j} U_{i}^{n}\left(\mathscr{R}_{\mathscr{M}}^{(i)}\left(u_{i}^{n}-\mathscr{P}_{\mathscr{E}}^{(i)}\left(U_{i}^{n}\right)\right)\right)_{K} \mathrm{~d} \boldsymbol{x}+R_{2,1}
\end{aligned}
$$

where the remainder $R_{2,1}$ is given by

$$
\begin{aligned}
R_{2,1}=\delta t \sum_{n=1}^{m} \int_{\Omega} & \left(r^{n}-\mathscr{P}_{\mathscr{M}}\left(r^{n}\right)\right) \boldsymbol{U}^{n} \cdot \nabla \boldsymbol{U}^{n} \cdot\left(\boldsymbol{u}^{n}-\mathscr{P}_{\mathscr{E}}\left(\boldsymbol{U}^{n}\right)\right) \mathrm{d} \boldsymbol{x} \\
& +\delta t \sum_{n=1}^{m} \int_{\Omega} \mathscr{P}_{\mathscr{M}}\left(r^{n}\right)\left(\boldsymbol{U}^{n}-\mathscr{R}_{\mathscr{M}} \mathscr{P}_{\mathscr{E}} \boldsymbol{U}^{n}\right) \cdot \nabla \boldsymbol{U}^{n} \cdot\left(\boldsymbol{u}^{n}-\mathscr{P}_{\mathscr{E}}\left(\boldsymbol{U}^{n}\right)\right) \mathrm{d} \boldsymbol{x} \\
& +\delta t \sum_{n=1}^{m} \int_{\Omega} \mathscr{P}_{\mathscr{M}}\left(r^{n}\right) \mathscr{R}_{\mathscr{M}} \mathscr{P}_{\mathscr{E}} \boldsymbol{U}^{n} \cdot \nabla \boldsymbol{U}^{n} \cdot\left(\boldsymbol{u}^{n}-\mathscr{P}_{\mathscr{E}}\left(\boldsymbol{U}^{n}\right)-\mathscr{R}_{\mathscr{M}}\left(\boldsymbol{u}^{n}-\mathscr{P}_{\mathscr{E}}\left(\boldsymbol{U}^{n}\right)\right)\right) \mathrm{d} \boldsymbol{x} .
\end{aligned}
$$

By virtue of (3.53), (3.54), (3.67) and (4.9) the remainder $R_{2,1}$ satisfies

$$
\left|R_{2,1}\right| \leq \operatorname{ch}_{\mathscr{M}},
$$

where the constant $c$ depends on $\bar{r},\|\nabla r\|_{L^{\infty}((0, T) \times \Omega)},\|\boldsymbol{U}\|_{L^{\infty}((0, T) \times \Omega)^{3}},\|\nabla \boldsymbol{U}\|_{L^{\infty}((0, T) \times \Omega)}$ and on $\mathscr{E}_{0, \mu}$. Using the Stoke's formula we infer that

$$
\begin{aligned}
\delta t \sum_{n=1}^{m} \sum_{i=1}^{3} \sum_{j=1}^{3} \sum_{K \in \mathscr{M}} \int_{K} r_{K}^{n}\left(\mathscr{R}_{\mathscr{M}}^{(j)} \mathscr{P}_{\mathscr{E}}^{(j)} U_{j}^{n}\right)_{K} \partial_{j} U_{i}^{n}\left(\mathscr{R}_{M^{(i)}}^{\left.\left(u_{i}^{n}-\mathscr{P}_{\mathscr{E}}^{(i)}\left(U_{i}^{n}\right)\right)\right)_{K} \mathrm{~d} \boldsymbol{x}}\right. \\
=\delta t \sum_{n=1}^{m} \sum_{i=1}^{3} \sum_{j=1}^{3} \sum_{K \in \mathscr{M}_{\sigma \in \mathscr{E}}} \sum_{(j)}\left[|\sigma| r_{K}^{n}\left(\mathscr{R}_{\mathscr{M}}^{(j)} \mathscr{P}_{\mathscr{E}}^{(j)} U_{j}^{n}\right)_{K}\right. \\
\left.\quad \times\left(\left(\mathscr{P}_{\mathscr{E}}^{(j)} U_{i}^{n}\right)_{\sigma}-\left(\mathscr{R}_{\mathscr{M}}^{(i)} \mathscr{P}_{\mathscr{E}}^{(i)} U_{i}^{n}\right)_{K}\right) \boldsymbol{e}^{(j)} \cdot \boldsymbol{n}_{\sigma, K}\left(\mathscr{R}_{\mathscr{M}}^{(i)}\left(u_{i}^{n}-\mathscr{P}_{\mathscr{E}}^{(i)}\left(U_{i}^{n}\right)\right)\right)_{K}\right],
\end{aligned}
$$

where we have used the identity

$$
\int_{K} \partial_{j} U_{i}^{n} \mathrm{~d} \boldsymbol{x}=\int_{K} \partial_{j}\left(U_{i}^{n}-\left(\mathscr{R}_{\mathscr{M}}^{(i)} \mathscr{P}_{\mathscr{E}}^{(i)} U_{i}^{n}\right)_{K}\right) \mathrm{d} \boldsymbol{x} .
$$

Finally keeping in mind the definition of the quantity $r_{\sigma}^{n \text {,up }}$ (see (5.8)) we obtain

$$
\begin{aligned}
& \delta t \sum_{n=1}^{m} \sum_{i=1}^{3} \sum_{j=1}^{3} \sum_{K \in \mathscr{M}_{\sigma \in \mathscr{E}} \sum^{(j)}(K)}\left[|\sigma| r_{K}^{n}\left(\mathscr{R}_{\mathscr{M}^{(j)}} \mathscr{P}_{\mathscr{E}}^{(j)} U_{j}^{n}\right)_{K}\left(\left(\mathscr{P}_{\mathscr{E}}^{(j)} U_{i}^{n}\right)_{\sigma}-\left(\mathscr{R}_{\mathscr{M}}^{(i)} \mathscr{P}_{\mathscr{E}}^{(i)} U_{i}^{n}\right)_{K}\right) \boldsymbol{e}^{(j)} \cdot \boldsymbol{n}_{\sigma, K}\right. \\
& \left.\times\left(\mathscr{R}_{\mathscr{M}}^{(i)}\left(u_{i}^{n}-\mathscr{P}_{\mathscr{E}}^{(i)}\left(U_{i}^{n}\right)\right)\right)_{K}\right] \\
& =\delta t \sum_{n=1}^{m} \sum_{i=1}^{3} \sum_{j=1}^{3} \sum_{K \in M^{\prime}} \sum_{\sigma \in \mathscr{E}_{\text {int }}^{(j)}(K)}\left[|\sigma| r_{\sigma}^{n, \mathrm{up}}\left(\mathscr{P}_{\mathscr{E}}^{(j)} U_{j}^{n}\right)_{\sigma}\left(\left(\mathscr{P}_{\mathscr{E}}^{(j)} U_{i}^{n}\right)_{\sigma}-\left(\mathscr{R}_{\mathscr{M}}^{(i)} \mathscr{P}_{\mathscr{E}}^{(i)} U_{i}^{n}\right)_{K}\right) \boldsymbol{e}^{(j)} \cdot \boldsymbol{n}_{\sigma, K}\right. \\
& \left.\times\left(\mathscr{R}_{\mathscr{M}}^{(i)}\left(u_{i}^{n}-\mathscr{P}_{\mathscr{E}}^{(i)}\left(U_{i}^{n}\right)\right)\right)_{K}\right]+R_{2,2},
\end{aligned}
$$

where by virtue of (3.49), (3.50) the remainder $R_{2,2}$ satisfies

$$
\left|R_{2,2}\right| \leq c \delta t \sum_{n=1}^{m} \int_{\Omega}\left\|\mathscr{P}_{\mathscr{E}} \boldsymbol{U}^{n}\right\|\left\|\mathscr{R}_{\mathscr{M}}\left(\boldsymbol{u}^{n}-\mathscr{P}_{\mathscr{E}}\left(\boldsymbol{U}^{n}\right)\right)\right\| \mathrm{d} \boldsymbol{x},
$$


where $c$ depends on $\|\nabla r\|_{L^{\infty}((0, T) \times \Omega)}$ and on $\|\boldsymbol{U}\|_{L^{\infty}((0, T) \times \Omega)^{3}}$. Consequently by virtue of (3.67) and (4.9)

$$
\left|R_{2,2}\right| \leq \operatorname{ch}_{\mathscr{M}}
$$

Now we write

$$
\begin{aligned}
& \delta t \sum_{n=1}^{m} \sum_{i=1}^{3} \sum_{j=1}^{3} \sum_{K \in \mathscr{M}} \sum_{\sigma \in \mathscr{E}_{\text {int }}^{(j)}}\left[|\sigma| r_{\sigma}^{n, \mathrm{up}}\left(\mathscr{P}_{\mathscr{E}}^{(j)} U_{j}^{n}\right)_{\sigma}\left(\left(\mathscr{P}_{\mathscr{E}}^{(j)} U_{i}^{n}\right)_{\sigma}-\left(\mathscr{R}_{\mathscr{M}^{(i)}}^{(i)} \mathscr{P}_{\mathscr{E}}^{(i)} U_{i}^{n}\right)_{K}\right) \boldsymbol{e}^{(j)} \cdot \boldsymbol{n}_{\sigma, K}\right. \\
& \left.\times\left(\mathscr{R}_{\mathscr{M}}^{(i)}\left(u_{i}^{n}-\mathscr{P}_{\mathscr{E}}^{(i)}\left(U_{i}^{n}\right)\right)\right)_{K}\right] \\
& =\delta t \sum_{n=1}^{m} \sum_{i=1}^{3} \sum_{j=1}^{3} \sum_{K \in \mathscr{M}} \sum_{\sigma \in \mathscr{E}_{\text {int }}^{(j)}(K)}\left[|\sigma| r_{\sigma}^{n, \text { up }}\left(\mathscr{P}_{\mathscr{E}}^{(j)} U_{j}^{n}\right)_{\sigma} \boldsymbol{e}^{(j)} \cdot \boldsymbol{n}_{\sigma, K}\right. \\
& \left.\times\left(\mathscr{R}_{\mathscr{E}}^{(i, j)}\left(u_{i}^{n}-\mathscr{P}_{\mathscr{E}}^{(i)} U_{i}^{n}\right)\right)_{\sigma}\left(\left(\mathscr{P}_{\mathscr{E}}^{(j)} U_{i}^{n}\right)_{\sigma}-\left(\mathscr{R}_{\mathscr{M}}^{(i)} \mathscr{P}_{\mathscr{E}}^{(i)} U_{i}^{n}\right)_{K}\right)\right]+R_{2,3},
\end{aligned}
$$

where by virtue of (3.49), (3.66), (3.67) and (4.9) the remainder $R_{2,3}$ satisfies

$$
\begin{aligned}
R_{2,3}=\delta t \sum_{n=1}^{m} \sum_{i=1}^{3} \sum_{j=1}^{3} \sum_{K \in \mathscr{M}} \sum_{\sigma \in \mathscr{C}_{\text {int }}^{(j)}(K)}\left[|\sigma| r_{\sigma}^{n, \text { up }}\left(\mathscr{P}_{\mathscr{E}}^{(j)} U_{j}^{n}\right)_{\sigma} \boldsymbol{e}^{(j)} \cdot \boldsymbol{n}_{\sigma, K}\left(\left(\mathscr{P}_{\mathscr{E}}^{(j)} U_{i}^{n}\right)_{\sigma}-\left(\mathscr{R}_{\mathscr{M}}^{(i)} \mathscr{P}_{\mathscr{E}}^{(i)} U_{i}^{n}\right)_{K}\right)\right. \\
\left.\times\left(\left(\mathscr{R}_{\mathscr{M}}^{(i)}\left(u_{i}^{n}-\mathscr{P}_{\mathscr{E}}^{(i)}\left(U_{i}^{n}\right)\right)\right)_{K}-\left(\mathscr{R}_{\mathscr{E}}^{(i, j)}\left(u_{i}^{n}-\mathscr{P}_{\mathscr{E}}^{(i)} U_{i}^{n}\right)\right)_{\sigma}\right)\right]
\end{aligned}
$$

Consequently

$$
\begin{aligned}
\left|R_{2,3}\right| \leq c \delta t \sum_{n=1}^{m} \sum_{i=1}^{3} \sum_{j=1}^{3} \sum_{K \in \mathscr{M}} \sum_{\sigma \in \mathscr{E}_{\text {int }}^{(j)}(K)}|\sigma| \mid\left(\mathscr{P}_{\mathscr{E}}^{(j)}\right. & \left.U_{i}^{n}\right)_{\sigma}-\left(\mathscr{R}_{\mathscr{M}}^{(i)} \mathscr{P}_{\mathscr{E}}^{(i)} U_{i}^{n}\right)_{K} \mid \\
& \times\left|\left(\mathscr{R}_{\mathscr{M}}^{(i)}\left(u_{i}^{n}-\mathscr{P}_{\mathscr{E}}^{(i)}\left(U_{i}^{n}\right)\right)\right)_{K}-\left(\mathscr{R}_{\mathscr{E}}^{(i, j)}\left(u_{i}^{n}-\mathscr{P}_{\mathscr{E}}^{(i)} U_{i}^{n}\right)\right)_{\sigma}\right|
\end{aligned}
$$

From (3.49) we infer that for any $(i, j) \in\{1,2,3\}^{2}, K \in \mathscr{M}, \sigma \in \mathscr{E}_{\text {int }}^{(j)}(K)$,

$$
\left|\left(\mathscr{P}_{\mathscr{E}}^{(j)} U_{i}^{n}\right)_{\sigma}-\left(\mathscr{R}_{\mathscr{M}}^{(i)} \mathscr{P}_{\mathscr{E}}^{(i)} U_{i}^{n}\right)_{K}\right| \leq c h_{K},
$$

which gives

$$
\begin{array}{r}
\left|R_{2,3}\right| \leq c \delta t \sum_{n=1}^{m} \sum_{i=1}^{3} \sum_{j=1}^{3} \sum_{K \in \mathscr{M}_{\sigma \in \mathscr{E}_{\text {int }}^{(j)}}} \sum_{K)}|\sigma| h_{\sigma}\left|\left(\mathscr{R}_{\mathscr{M}}^{(i)}\left(u_{i}^{n}-\mathscr{P}_{\mathscr{E}}^{(i)}\left(U_{i}^{n}\right)\right)\right)_{K}-\left(\mathscr{R}_{\mathscr{E}}^{(i, j)}\left(u_{i}^{n}-\mathscr{P}_{\mathscr{E}}^{(i)} U_{i}^{n}\right)\right)_{\sigma}\right| \\
\quad \leq c h_{\mathscr{M}} \delta t \sum_{n=1}^{m} \sum_{i=1}^{3} \sum_{j=1}^{3} \sum_{K \in \mathscr{M}_{\sigma \in \mathscr{E}_{\text {int }}^{(j)}}(K)}\left\|\partial_{j}\left(u_{i}^{n}-\mathscr{P}_{\mathscr{E}}^{(i)} U_{i}^{n}\right)\right\|_{L^{1}\left(D_{\sigma}\right)} .
\end{array}
$$

The latter inequality yields, by virtue of (4.9) and (3.55),

$$
\left|R_{2,3}\right| \leq \operatorname{ch}_{\mathscr{M}}
$$

where $c$ depends on $\bar{r},\left\|\boldsymbol{U}_{L^{\infty}((0, T) \times \Omega)^{3}},\right\| \nabla r\left\|_{L^{\infty}((0, T) \times \Omega)},\right\| \nabla \boldsymbol{U}_{L^{\infty}((0, T) \times \Omega)}, \mathscr{E}_{0, \mathscr{M}}$ and on $\eta_{\mathscr{M}}$. Consequently

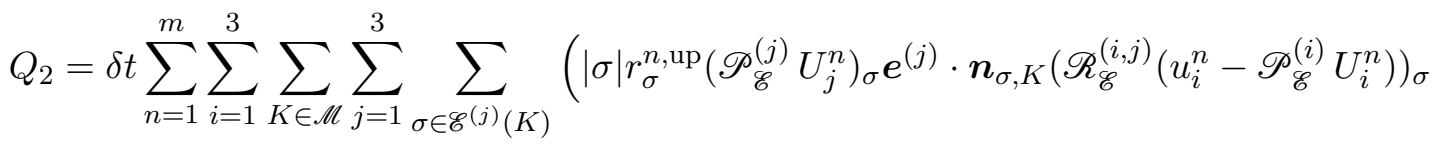

$$
\begin{aligned}
& \left.\times\left(\left(\mathscr{P}_{\mathscr{E}}^{(j)} U_{i}^{n}\right)_{\sigma}-\left(\mathscr{R}_{\mathscr{M}}^{(i)} \mathscr{P}_{\mathscr{E}}^{(i)} U_{i}^{n}\right)_{K}\right)\right)+R_{2}
\end{aligned}
$$

where the remainder $R_{2}$ satisfies,

$$
\left|R_{2}\right| \leq \operatorname{ch}_{\mathscr{M}}
$$

Summing (6.3) and (6.6) we obtain the expected result, that is formula (6.1). 


\section{End of the proof of the error estimate Theorem 3.2}

In this Section we put together the relative energy inequality (5.14) and the identity (6.1) derived in the previous section to obtain a discrete version of inequality (2.16). The final inequality resulting from this manipulation is formulated in the following lemma.

Lemma 7.1. Under assumptions of Theorem 3.2 there exists $c$ depending on parameters (5.11-5.13) such that for all $m=1, \ldots, N$, there holds:

$$
\begin{gathered}
\mathfrak{E}\left(\varrho^{m}, \boldsymbol{u}^{m} \mid r_{\mathscr{M}}^{m}, \boldsymbol{U}_{\mathscr{E}}^{m}\right)+\delta t \frac{\mu}{2} \sum_{n=1}^{m}\left\|\boldsymbol{u}^{n}-\boldsymbol{U}_{\mathscr{E}}^{n}\right\|_{1, \mathscr{E}, 0}^{2} \\
\leq c\left[h_{\mathscr{M}}^{A}+\sqrt{\delta t}+\mathscr{E}\left(\varrho^{0}, \boldsymbol{u}^{0} \mid r_{\mathscr{M}}^{0}, \boldsymbol{U}_{\mathscr{E}}^{0}\right)\right]+c \delta t \sum_{n=1}^{m} \mathfrak{E}\left(\varrho^{n}, \boldsymbol{u}^{n} \mid r_{\mathscr{M}}^{n}, \boldsymbol{U}_{\mathscr{E}}^{n}\right),
\end{gathered}
$$

where $A$ is defined in (3.79).

Proof. Gathering the formulae (5.14) and (6.1), one gets

$$
\mathfrak{E}\left(\varrho^{m}, \boldsymbol{u}^{m} \mid r_{\mathscr{M}}^{m}, U_{\mathscr{C}}^{m}\right)-\mathfrak{E}\left(\varrho^{0}, \boldsymbol{u}^{0} \mid r_{\mathscr{M}}^{0}, U_{\mathscr{C}}^{0}\right)+\mu \delta t \sum_{n=1}^{m}\left\|\boldsymbol{u}^{n}-\boldsymbol{U}_{\mathscr{C}}^{n}\right\|_{1, \mathscr{C}, 0}^{2} \leq \mathscr{P}_{1}+\mathscr{P}_{2}+\mathscr{P}_{3}+\mathscr{Q},
$$

where

$$
\begin{aligned}
& \mathscr{P}_{1}=\delta t \sum_{n=1}^{m} \int_{\Omega}\left(\varrho^{n-1}-r_{\mathscr{M}}^{n-1}\right) \frac{\boldsymbol{U}_{\mathscr{E}}^{n}-\boldsymbol{U}_{\mathscr{E}}^{n-1}}{\delta t} \cdot\left(\boldsymbol{U}_{\mathscr{E}}^{n}-\boldsymbol{u}^{n}\right) \mathrm{d} \boldsymbol{x},
\end{aligned}
$$

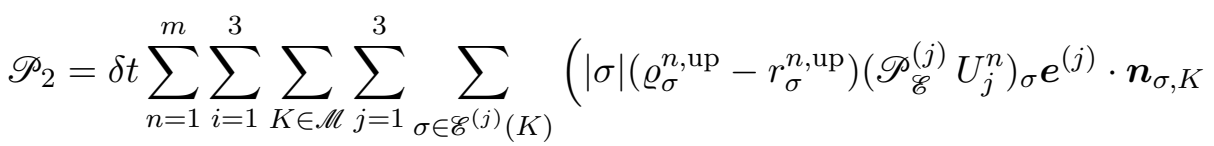

$$
\begin{aligned}
& \left.\times\left(\mathscr{R}_{\mathscr{E}}^{(i, j)}\left(\mathscr{P}_{\mathscr{E}}^{(i)}\left(U_{i}^{n}\right)-u_{i}^{n}\right)\right)_{\sigma}\left(\left(\mathscr{R}_{\mathscr{M}}^{(i)} \mathscr{P}_{\mathscr{E}}^{(i)} U_{i}^{n}\right)_{K}-\left(\mathscr{P}_{\mathscr{E}}^{(j)} U_{i}^{n}\right)_{\sigma}\right)\right), \\
& \mathscr{P}_{3}=\delta t \sum_{n=1}^{m} \int_{\Omega}\left(p\left(r_{\mathscr{M}}^{n}\right)-p\left(\varrho^{n}\right)\right) \operatorname{div} \boldsymbol{U}^{n} \mathrm{~d} \boldsymbol{x} \\
& +\delta t \sum_{n=1}^{m} \int_{\Omega}\left(\frac{r_{\mathscr{M}}^{n}-\varrho^{n}}{r_{\mathscr{M}}^{n}} p^{\prime}\left(r_{\mathscr{M}}^{n}\right) \mathscr{R}_{\mathscr{M}}\left(\boldsymbol{u}^{n}\right) \cdot \nabla r^{n}+\frac{r_{\mathscr{M}}^{n}-\varrho_{\mathscr{M}}^{n}}{r_{\mathscr{M}}^{n}} p^{\prime}\left(r_{\mathscr{M}}^{n}\right)\left[\partial_{t} r\right]^{n}\right) \mathrm{d} \boldsymbol{x}, \\
& \mathbb{Q}=\mathscr{R}_{\mathscr{M}, \delta t}^{m}+\mathscr{S}_{\mathscr{M}, \delta t}^{m}+\mathscr{K}_{\mathscr{M}, \delta t}^{m},
\end{aligned}
$$

and where the remainders $\mathscr{R}_{\mathscr{M}, \delta t}^{m}, \mathscr{G}_{\mathscr{M}, \delta t}^{m}$ and $\mathscr{K}_{\mathscr{M}, \delta t}^{m}$ are explicited in Lemma 5.1 and Lemma 6.1.

Step 1: Term $\mathscr{P}_{1}$. Writing $\mathscr{P}_{1}=\delta t \sum_{n=1}^{m} \mathscr{P}_{1}^{n}$, an application of the Taylor formula and of Lemma 2.1 gives, since $\gamma \geq \frac{6}{5}$ :

$$
\begin{aligned}
&\left|\mathscr{P}_{1}^{n}\right| \leq c \int_{\Omega} \mid \varrho^{n-1}- r_{\mathscr{M}}^{n-1} \| \boldsymbol{u}^{n}-\boldsymbol{U}_{\mathscr{E}}^{n} \mid \mathrm{d} \boldsymbol{x} \\
& \quad \leq c \int_{\Omega}\left(\sqrt{E\left(\varrho^{n-1} \mid r_{\mathscr{M}}^{n-1}\right)}+\left(E\left(\varrho^{n-1} \mid r_{\mathscr{M}}^{n-1}\right)\right)^{5 / 6}\right)\left|\boldsymbol{u}^{n}-\boldsymbol{U}_{\mathscr{E}}^{n}\right| \mathrm{d} \boldsymbol{x} \\
& \quad \leq c\left(\int_{\Omega} E\left(\varrho^{n-1} \mid r_{\mathscr{M}}^{n-1}\right)^{3 / 5}+E\left(\varrho^{n-1} \mid r_{\mathscr{M}}^{n-1}\right) \mathrm{d} \boldsymbol{x}\right)^{5 / 6}\left\|\boldsymbol{u}^{n}-\boldsymbol{U}_{\mathscr{E}}^{n}\right\|_{L^{6}\left(\Omega, \mathbb{R}^{3}\right)} \\
& \leq \frac{c}{\delta}\left[\left(\int_{\Omega}\left(E\left(\varrho^{n-1} \mid r_{\mathscr{M}}^{n-1}\right) \mathrm{d} \boldsymbol{x}\right)^{5 / 3}+\int_{\Omega} E\left(\varrho^{n-1} \mid r_{\mathscr{M}}^{n-1}\right)\right) \mathrm{d} \boldsymbol{x}\right]+\delta\left\|\boldsymbol{u}^{n}-\boldsymbol{U}_{\mathscr{E}}^{n}\right\|_{L^{6}\left(\Omega, \mathbb{R}^{3}\right)}^{2} \\
& \quad \leq \frac{c}{\delta} \mathfrak{E}\left(\varrho^{n-1}, \boldsymbol{u}^{n-1} \mid r_{\mathscr{M}}^{n-1}, \boldsymbol{U}_{\mathscr{E}}^{n-1}\right)+\delta\left\|\boldsymbol{u}^{n}-\boldsymbol{U}_{\mathscr{E}}^{n}\right\|_{1, \mathscr{E}, 0}^{2},
\end{aligned}
$$

with any $\delta>0$, where $c$ depends on $\underline{r}, \bar{r},\left\|\partial_{t} \boldsymbol{U}\right\|_{L^{\infty}((0, T) \times \Omega)^{3}}$. Here we have used Lemma 3.2 to get a bound on $\left\|\boldsymbol{u}^{n}-\boldsymbol{U}_{\mathscr{E}}^{n}\right\|_{L^{6}(\Omega)^{3}}^{2}$ by $\left\|\boldsymbol{u}^{n}-\boldsymbol{U}_{\mathscr{E}}^{n}\right\|_{1, \mathscr{\ell}, 0}^{2}$, the Jensen inequality and the Young inequality to perform the before last inequality. Consequently

$$
\left|\mathscr{P}_{1}\right| \leq \frac{c}{\delta}\left(\mathfrak{E}\left(\varrho^{0}, r_{\mathscr{M}}^{0} \mid \boldsymbol{u}^{0}, \boldsymbol{U}_{\mathscr{E}}^{0}\right)+\delta t \sum_{n=1}^{m} \mathfrak{E}\left(\varrho^{n}, \boldsymbol{u}^{n} \mid r_{\mathscr{M}}^{n}, \boldsymbol{U}_{\mathscr{E}}^{n}\right)\right)+\delta \delta t \sum_{n=1}^{m}\left\|\boldsymbol{u}^{n}-\boldsymbol{U}_{\mathscr{E}}^{n}\right\|_{1, \mathscr{E}, 0}^{2} .
$$


Step 2: Term $\mathscr{P}_{2}$. We write $\mathscr{P}_{2}=\delta t \sum_{n=1}^{m} \mathscr{P}_{2}^{n}$ where Lemma 2.1, the Hölder inequality yield, since $\gamma \geq \frac{3}{2}$

$$
\begin{aligned}
& \left|\mathscr{P}_{2}^{n}\right| \leq c \sum_{i=1}^{3} \sum_{j=1}^{3} \sum_{K \in \mathscr{M}_{\sigma \in \mathscr{E}} \sum_{(j)}(K) \cap \mathscr{E}_{\text {int }}} h_{K}|\sigma|\left|\varrho_{\sigma}^{n, \text { up }}-r_{\sigma}^{n, \text { up }} \|\left(\mathscr{R}_{\mathscr{E}}^{(i, j)}\left(u_{i}^{n}-\mathscr{P}_{\mathscr{E}}^{(i)}\left(U_{i}^{n}\right)\right)\right)_{\sigma}\right| \\
& \leq c\left[\left(\sum_{K \in \mathscr{M}} \sum_{\sigma \in \mathscr{E}(K) \cap \mathscr{E}_{\text {int }}}|\sigma| h_{\sigma}\left(E\left(\varrho_{\sigma}^{n, \text { up }} \mid r_{\sigma}^{n, \text { up }}\right)\right)^{1 / 2}+\right.\right. \\
& \left.\left(\sum_{K \in \mathscr{M}} \sum_{\sigma \in \mathscr{E}(K) \cap \mathscr{E}_{\mathrm{int}}}|\sigma| h_{\sigma} E\left(\varrho_{\sigma}^{n, \mathrm{up}} \mid r_{\sigma}^{n, \mathrm{up}}\right)\right)^{2 / 3}\right] \\
& \times\left(\sum_{i=1}^{3} \sum_{j=1}^{3} \sum_{K \in \mathscr{M}_{\sigma \in \mathscr{E}_{\text {int }}^{(j)}}} \sum_{\sigma)} h_{\sigma}|\sigma|\left|\left(\mathscr{R}_{\mathscr{E}}^{(i, j)}\left(u_{i}^{n}-\mathscr{P}_{\mathscr{E}}^{(i)}\left(U_{i}^{n}\right)\right)\right)_{\sigma}\right|^{6}\right)^{1 / 6}
\end{aligned}
$$

where $c$ depends on $\|\boldsymbol{U}\|_{L^{\infty}((0, T) \times \Omega)^{3}},\|\nabla \boldsymbol{U}\|_{L^{\infty}((0, T) \times \Omega)^{9}}$ and on $\eta_{\mathscr{M}}$. Next, we observe that the contribution of the face $\sigma=K \mid L$ to the sums $\sum_{K \in \mathscr{M}} \sum_{\sigma \in \mathscr{C}(K)}|\sigma| h_{\sigma} E\left(\varrho_{\sigma}^{n \text {,up }} \mid r_{\sigma}^{n \text {,up }}\right)$ is less or equal than $2|\sigma| h_{\sigma}\left(E\left(\varrho_{K}^{n} \mid r_{K}^{n}\right)+E\left(\varrho_{L}^{n} \mid r_{L}^{n}\right)\right)$. Moreover using (3.59) and (3.65) we have

$$
\left(\sum_{i=1}^{3} \sum_{j=1}^{3} \sum_{K \in \mathscr{M}_{\sigma \in \mathscr{C}_{\text {int }}^{(j)}}} \sum_{K)} h_{K}\left|\sigma \|\left(\mathscr{R}_{\mathscr{E}}^{(i, j)}\left(u_{i}^{n}-\mathscr{P}_{\mathscr{E}}^{(i)}\left(U_{i}^{n}\right)\right)\right)_{\sigma}\right|^{6}\right)^{1 / 6} \leq c\left\|\boldsymbol{u}^{n}-\boldsymbol{U}_{\mathscr{E}}^{n}\right\|_{L^{6}(\Omega)^{3}} \leq c\left\|\boldsymbol{u}^{n}-\boldsymbol{U}_{\mathscr{E}}^{n}\right\|_{1, \mathscr{E}, 0}
$$

where the constant $c$ depends on $\eta_{\mathscr{M}}$ in a nondecreasing way.

Consequently, we get by the same reasoning as in the previous step, under assumption $\gamma \geq 3 / 2$,

$$
\left|\mathscr{P}_{2}\right| \leq \frac{c}{\delta} \delta t \sum_{n=1}^{m} \mathfrak{E}\left(\varrho^{n}, \boldsymbol{u}^{n} \mid r_{\mathscr{M}}^{n}, \boldsymbol{U}_{\mathscr{E}}^{n}\right)+\delta \delta t \sum_{n=1}^{m}\left\|\boldsymbol{u}^{n}-\boldsymbol{U}_{\mathscr{C}}^{n}\right\|_{1, \mathscr{E}, 0}^{2}
$$

where $c$ depends on $\underline{r}, \bar{r}\|\boldsymbol{U}\|_{L^{\infty}((0, T) \times \Omega)^{3}},\|\nabla \boldsymbol{U}\|_{L^{\infty}((0, T) \times \Omega)^{9}}$ and $\eta_{\mathscr{M}}$ in a nondecreasing way.

Step 3: Term $\mathscr{P}_{3}$. Since the pair $(r, \boldsymbol{U})$ satisfies continuity equation (1.1a) in the classical sense, we have for all $n=1, \ldots, N$,

$$
\left[\partial_{t} r\right]^{n}+\boldsymbol{U}^{n} \cdot \nabla r^{n}=-r^{n} \operatorname{div} \boldsymbol{U}^{n},
$$

where we recall that $\left[\partial_{t} r\right]^{n}(x)=\partial_{t} r\left(t^{n}, x\right)$ in accordance with (3.77). Using this identity, we write

$$
\begin{aligned}
& \mathscr{P}_{3}=\mathscr{P}_{3,1}+\mathscr{P}_{3,2}+\mathscr{P}_{3,3}, \quad \mathscr{P}_{3, i}=\delta t \sum_{n=1}^{m} \mathscr{P}_{3, i}^{n}, \\
& \text { with } \mathscr{P}_{3,1}^{n}=-\int_{\Omega}\left(p\left(\varrho^{n}\right)-p^{\prime}\left(r_{\mathscr{M}}^{n}\right)\left(\varrho^{n}-r_{\mathscr{M}}^{n}\right)-p\left(r_{\mathscr{M}}^{n}\right)\right) \operatorname{div} \boldsymbol{U}^{n} \mathrm{~d} \boldsymbol{x} \\
& \mathscr{P}_{3,2}^{n}=\int_{\Omega} \frac{r_{\mathscr{M}}^{n}-\varrho^{n}}{r_{\mathscr{M}}^{n}} p^{\prime}\left(r_{\mathscr{M}}^{n}\right)\left(\mathscr{R}_{\mathscr{M}}\left(\boldsymbol{u}^{n}\right)-\boldsymbol{U}^{n}\right) \cdot \nabla r^{n} \mathrm{~d} \boldsymbol{x}, \\
& \text { and } \mathscr{P}_{3,3}^{n}=\int_{\Omega} \frac{r_{\mathscr{M}}^{n}-\varrho^{n}}{r_{\mathscr{M}}^{n}} p^{\prime}\left(r_{\mathscr{M}}^{n}\right)\left(r_{\mathscr{M}}^{n}-r^{n}\right) \operatorname{div} \boldsymbol{U}^{n} \mathrm{~d} \boldsymbol{x} .
\end{aligned}
$$

From the asympototic behaviour (1.5) for large values of density and Lemma 2.1 we easily deduce that

$$
\left|\mathscr{P}_{3,1}\right| \leq c \delta t \sum_{n=1}^{m} \mathfrak{E}\left(\varrho^{n}, \boldsymbol{u}^{n} \mid r_{\mathscr{M}}^{n}, \boldsymbol{U}_{\mathscr{C}}^{n}\right)
$$

where $c$ depends on $\underline{r}, \bar{r}, \min _{[\underline{r}, \bar{r}]} p, \min _{[\underline{r} / 2,2 \bar{r}]} p^{\prime}$ and $\|\nabla \boldsymbol{U}\|_{L^{\infty}((0, T) \times \Omega)}$. From the total mass conservation (3.16) and (3.50) we deduce

$$
\left|\mathscr{P}_{3,3}\right| \leq \operatorname{ch}_{\mathscr{M}},
$$

where $c$ depends on $\underline{r}, \bar{r},\|\nabla r\|_{L^{\infty}((0, T) \times \Omega)^{3}},\|\nabla \boldsymbol{U}\|_{L^{\infty}((0, T) \times \Omega)^{3}}$ and on $\mathscr{E}_{0, \mathscr{M}}$. 
Last but not least, the same reasoning as in Step 2 leads to the estimate

$$
\left|\mathscr{P}_{3,2}\right| \leq \frac{c}{\delta}\left(h_{\mathscr{M}}+\delta t \sum_{n=1}^{m} \mathfrak{E}\left(\varrho^{n}, \boldsymbol{u}^{n} \mid r_{\mathscr{M}}^{n}, \boldsymbol{U}_{\mathscr{E}}^{n}\right)\right)+\delta \delta t \sum_{n=1}^{m}\left\|\boldsymbol{u}^{n}-\boldsymbol{U}_{\mathscr{E}}^{n}\right\|_{1, \mathscr{E}, 0}^{2} .
$$

with any $\delta>0$, where $c$ depends on $\underline{r}, \bar{r},\|\nabla r\|_{L^{\infty}((0, T) \times \Omega)^{3}},\|\nabla \boldsymbol{U}\|_{L^{\infty}((0, T) \times \Omega)^{3}}$ and on $\mathscr{E}_{0, \mathcal{M}}$ in a non increasing way. Gathering the formulae (7.1)-(7.6) with $\delta$ sufficiently small (with respect to $\mu$ ), we conclude the proof of Lemma 7.1.

Finally, Lemma 7.1 in combination with the bound (4.14) yields

$$
\mathfrak{E}\left(\varrho^{m}, \boldsymbol{u}^{m} \mid r_{\mathscr{M}}^{m}, \boldsymbol{U}_{\mathscr{C}}^{m}\right) \leq c\left(h_{\mathscr{M}}^{A}+\delta t+\mathfrak{E}\left(\varrho^{0}, \boldsymbol{u}^{0} \mid r_{\mathscr{M}}^{0}, \boldsymbol{U}_{\mathscr{C}}^{0}\right)\right)+c \delta t \sum_{n=1}^{m-1} \mathfrak{E}\left(\varrho^{n}, \boldsymbol{u}^{n} \mid r_{\mathscr{M}}^{n}, \boldsymbol{U}_{\mathscr{C}}^{n}\right)
$$

whence Theorem 3.2 is a direct consequence of the standard discrete version of Gronwall's lemma. Theorem 3.2 is thus proved.

\section{A Existence of a discrete solution}

This section is devoted to the proof of Theorem 3.1. Indeed, Theorem 3.1 is an easy consequence of Proposition A.1 below.

Proposition A.1. Consider a $M A C$ grid $\mathscr{D}=(\mathscr{M}, \mathscr{E})$ of $\Omega$ of size $h_{\mathscr{M}}$. Let $\delta t>0$. Let $p: \mathbb{R} \rightarrow \mathbb{R}$ such that $p \in C^{1}\left(\mathbb{R}_{+}^{\star}\right)$. Let $\left(\varrho^{\star}, \boldsymbol{u}^{\star}\right) \in L_{\mathscr{M}} \times \mathbf{H}_{\mathscr{E}, 0}$ such that $\varrho^{\star}>0$ a.e in $\Omega$. Then there exists $(\varrho, \boldsymbol{u}) \in$ $L_{\mathscr{M}} \times \mathbf{H}_{\mathscr{E}, 0}$ such that $\varrho>0$ a.e in $\Omega$ which satisfies

$$
\begin{aligned}
& \frac{1}{\delta t}\left(\varrho-\varrho^{\star}\right)+\operatorname{div}_{\mathscr{M}}^{\text {up }}(\varrho \boldsymbol{u})=0, \\
& \frac{1}{\delta t}\left(\varrho^{(i)} u_{i}-\widehat{\varrho}^{\star}(i) u_{i}^{\star}\right)+\operatorname{div}_{\mathscr{E}}^{(i)}\left(\varrho \boldsymbol{u} u_{i}\right)-\mu \Delta_{\mathscr{E}}^{(i)} u_{i} \\
& -(\mu+\lambda) \Im_{i} \operatorname{div}_{\mathscr{M}} \boldsymbol{u}+\widetilde{\partial}_{i} p(\varrho)=0, \forall i=1, \ldots d .
\end{aligned}
$$

Let us state the abstract theorem which will be used hereafter to prove Proposition A.1.

Theorem A.1. Let $N$ and $M$ be two positive integers. Let $\epsilon, C_{1}$ and $C_{2}$ three real numbers with $0<\epsilon<C_{2}, C_{1}>0$. Let $\|\cdot\|$ be a norm over $\mathbb{R}^{N}$. One defines $V$ and $W$ as follows:

$$
\begin{gathered}
V=\left\{(x, y) \in \mathbb{R}^{N} \times \mathbb{R}^{M}, y>0\right\}, \\
W=\left\{(x, y) \in \mathbb{R}^{N} \times \mathbb{R}^{M},\|x\|<C_{1} \text { and } \epsilon<y<C_{2}\right\},
\end{gathered}
$$

where, for any real number $c$, the notation $y>c$ means that each component of $y$ is greater than $c$. Let $F$ be a continuous function from $V \times[0,1]$ to $\mathbb{R}^{N} \times \mathbb{R}^{M}$ satisfying:

1. $\forall \zeta \in[0,1]$, if $v \in V$ is such that $F(v, \zeta)=0$ then $v \in W$,

2. The equation $F(v, 0)=0$ is a linear system on $v$ and has a solution in $W$.

Then there exists at least a solution $v \in W$ such that $F(v, 1)=0$.

Theorem A.1 is an easy consequence of the topological degree theory (see, for instance [8]). Indeed, $F$ is a continuous mapping from $\bar{W} \times[0,1]$ to $\mathbb{R}^{N} \times \mathbb{R}^{M}$. The set $W$ is a bounded open subset of $\mathbb{R}^{N} \times \mathbb{R}^{M}$. Hypothesis 1 gives that the degree of the application $F(\cdot, \zeta)$ on the set $W$ with point 0 is independent of $\zeta$, for $\zeta \in[0,1]$ (this is due to the fact that $F(v, \zeta)=0$ has no solution on the boundary of $W$ ). Hypothesis 2 gives that the degree of the application $F(\cdot, 0)$ on the set $W$ with point 0 is non zero. The conclusion is that the degree of the application $F(\cdot, 1)$ on the set $W$ with point 0 is non zero and this gives that there exists $v \in W$ such that $F(v, 1)=0$. 
Proof. The proof Proposition A.1 uses Theorem A.1. First, we remark that an element $\boldsymbol{v}$ of $\mathbf{H}_{\mathscr{E}, 0}$ can be identified to its degrees of freedom, namely the set of $v_{\sigma}, \sigma \in \mathscr{E}_{\text {int }}^{(i)}, i \in[1, d]$, so that $\mathbf{H}_{\mathscr{E}, 0}$ is identified to $\mathbb{R}^{N}$ where $N$ is the number of degrees of freedom. Similarly an element $q$ of $L_{\mathscr{M}}$ can be identified to its degrees of freedom, namely the set of $q_{K}, K \in \mathscr{M}$, so that $L_{\mathscr{M}}$ is identified to $\mathbb{R}^{M}$ where $M$ is the number of degrees of freedom.

Let us define

$$
V=\left\{(\boldsymbol{u}, \varrho) \in \mathbf{H}_{\mathscr{\delta}, 0} \times L_{\mathscr{M}}, \varrho_{K}>0 \forall K \in \mathscr{M}\right\} .
$$

and consider the mapping

$$
\begin{aligned}
F: & V \times[0,1] \longrightarrow \mathbf{H}_{\mathscr{E}, 0} \times L_{\mathscr{M}} \\
& (\boldsymbol{u}, \varrho, \zeta) \mapsto F(\boldsymbol{u}, \varrho, \zeta)=(\hat{\boldsymbol{u}}, \hat{\varrho}),
\end{aligned}
$$

where $(\hat{\boldsymbol{u}}, \hat{\varrho}) \in \mathbf{H}_{\mathscr{C}, 0} \times L_{\mathscr{M}}$ is such that

$$
\begin{aligned}
\int_{\Omega} \hat{\boldsymbol{u}} \cdot \boldsymbol{v} \mathrm{d} \boldsymbol{x}= & \int_{\Omega} \frac{\varrho \boldsymbol{u}-\varrho^{\star} \boldsymbol{u}^{\star}}{\delta t} \cdot \boldsymbol{v} \mathrm{d} \boldsymbol{x}+\mu[\boldsymbol{u}, \boldsymbol{v}]_{1, \mathscr{E}, 0}+(\mu+\lambda) \int_{\Omega} \operatorname{div}_{\mathscr{M}} \boldsymbol{u} \operatorname{div} \mathscr{M} \boldsymbol{v} \mathrm{d} \boldsymbol{x} \\
& +\zeta \int_{\Omega} \operatorname{div}_{\mathscr{E}}(\varrho \boldsymbol{u} \otimes \boldsymbol{u}) \cdot \boldsymbol{v} \mathrm{d} \boldsymbol{x}-\zeta \int_{\Omega} p(\varrho) \operatorname{div}_{\mathscr{M}} \boldsymbol{v} \mathrm{d} \boldsymbol{x}, \forall \boldsymbol{v} \in \mathbf{H}_{\mathscr{E}, 0}, \\
\int_{\Omega} \hat{\varrho} q \mathrm{~d} \boldsymbol{x}= & \int_{\Omega} \frac{\varrho-\varrho^{\star}}{\delta t} q \mathrm{~d} \boldsymbol{x}+\zeta \int_{\Omega} \operatorname{div}_{\mathscr{M}}^{\mathrm{up}}(\varrho \boldsymbol{u}) q \mathrm{~d} \boldsymbol{x}, \forall q \in L_{\mathscr{M}} .
\end{aligned}
$$

Any solution of $F(\boldsymbol{u}, \varrho, 1)=0$ is a solution of Problem A.1. Note also that in $(A .2 a)$ the fluxes $F_{\epsilon, \sigma}(\varrho, \boldsymbol{u})$ which determine $\operatorname{div}_{\mathscr{E}}(\varrho \boldsymbol{u} \otimes \boldsymbol{u})$ are constructed from the fluxes $F_{\sigma, K}(\varrho, \boldsymbol{u})$ which determine $\operatorname{div}_{\mathscr{M}}^{\mathrm{up}}(\varrho \boldsymbol{u})$ as in (3.19) and (3.20).

It is easily checked that $F$ is indeed a one to one mapping, since the values of $\hat{u}_{i} ; i=1, \cdots, d$, and $\hat{\varrho}$ are readily obtained by setting in this system $v_{i}=1_{D_{\sigma}}, v_{j}=0, j \neq i$ in (A.2a) and $q=1_{K}$ in (A.2b). Moreover, the mapping $F$ is clearly continuous.

Let $(\boldsymbol{u}, \varrho) \in \mathbf{H}_{\mathscr{E}, 0} \times L_{\mathscr{M}}$ and $\zeta \in[0,1]$ such that $F(\boldsymbol{u}, \varrho, \zeta)=(0,0)$ (in particular $\varrho>0$ ). Then for any $(\boldsymbol{v}, q) \in \mathbf{H}_{\mathscr{E}, 0} \times L_{\mathscr{M}}$,

$$
\begin{aligned}
\int_{\Omega} \frac{\varrho \boldsymbol{u}-\varrho^{\star} \boldsymbol{u}^{\star}}{\delta t} & \cdot \boldsymbol{v} \mathrm{d} \boldsymbol{x}+\zeta \int_{\Omega} \operatorname{div} \mathscr{E}(\varrho \boldsymbol{u} \otimes \boldsymbol{u}) \cdot \boldsymbol{v} \mathrm{d} \boldsymbol{x}+\mu[\boldsymbol{u}, \boldsymbol{v}]_{1, \mathscr{E}, 0} \\
& +(\mu+\lambda) \int_{\Omega} \operatorname{div} \mathscr{M} \boldsymbol{u} \operatorname{div} \mathscr{M} \boldsymbol{v} \mathrm{d} \boldsymbol{x}-\zeta \int_{\Omega} p(\varrho) \operatorname{div}_{\mathscr{M}} \boldsymbol{v} \mathrm{d} \boldsymbol{x}=0 \\
\int_{\Omega} \frac{\varrho-\varrho^{\star}}{\delta t} q \mathrm{~d} \boldsymbol{x} & +\zeta \int_{\Omega} \operatorname{div}_{\mathscr{M}}^{\mathrm{up}}(\varrho \boldsymbol{u}) q \mathrm{~d} \boldsymbol{x}=0 .
\end{aligned}
$$

Taking $q=1$ as a test function in $(A .3 b)$, and using the conservativity of the fluxes we obtain

$$
\int_{\Omega} \varrho \mathrm{d} \boldsymbol{x}=\|\varrho\|_{L^{1}(\Omega)}=\int_{\Omega} \varrho^{\star} \mathrm{d} \boldsymbol{x}>0 .
$$

This relation provides a bound for $\varrho$ in the $L^{1}$ norm, and therefore in all norms since the problem is of finite dimension.

Taking $\boldsymbol{u}$ as a test function in $(A .3 a)$ and following the proof of Theorem 4.1 gives

$$
\|\boldsymbol{u}\|_{1, \mathscr{E}, 0}<C_{1}
$$

where $C_{1}$ depends only on the data of the problem. Now a straightforward computation gives

$$
\varrho_{K} \geq \frac{\min _{K \in \mathscr{M}}|K| \min _{K \in \mathscr{M}} \varrho_{K}^{\star}}{|\Omega|+\delta t \sum_{\sigma \in \mathscr{E}_{\mathrm{int}}, \sigma=K \mid L}\left|u_{\sigma, K}\right|} .
$$

Consequently by virtue of $(A .5)$ there exists $\epsilon>0$ such that

$$
\varrho_{K}>\epsilon, \forall K \in \mathscr{M}
$$


where $\epsilon$ depends only on the data of the problem. Clearly from (A.4) one has also

$$
\varrho_{K} \leq \frac{\int_{\Omega} \varrho^{\star} \mathrm{d} \boldsymbol{x}}{\min _{K \in \mathscr{M}}|K|} \forall K \in \mathscr{M}
$$

Then, there exists $C_{2}>0$ such that

$$
\varrho_{K}<C_{2} \forall K \in \mathscr{M} \text {. }
$$

This shows that Hypothesis 1 of Theorem A.1 is satisfied with $W$ defined by

$$
W=\left\{(\boldsymbol{u}, \varrho) \in \mathbf{H}_{\mathscr{C}, 0} \times L_{\mathscr{M}} \text { such that }\|\boldsymbol{u}\|<C_{1}, \epsilon<\varrho<C_{2}\right\} .
$$

We prove now that Hypothesis 2 is satisfied. Let $\zeta=0$ the system $F(\boldsymbol{u}, \varrho, 0)=0$ reads:

$$
\begin{aligned}
& \int_{\Omega} \frac{\varrho \boldsymbol{u}-\varrho^{\star} \boldsymbol{u}^{\star}}{\delta t} \cdot \boldsymbol{v} \mathrm{d} \boldsymbol{x}+\mu[\boldsymbol{u}, \boldsymbol{v}]_{1, \mathscr{\ell}, 0}+(\mu+\lambda) \int_{\Omega} \operatorname{div}_{\mathscr{M}} \boldsymbol{u} \operatorname{div}_{\mathscr{M}} \boldsymbol{v} \mathrm{d} \boldsymbol{x}=0, \forall \boldsymbol{v} \in \mathbf{H}_{\mathscr{C}, 0}, \\
& \varrho_{K}=\varrho_{K}^{\star}, \forall K \in \mathscr{M} .
\end{aligned}
$$

Equation (A.8b) gives $\rho$ and Equation (A.8a) is a linear system on $u$ which has clearly one and only one solution. This solution $(u, \rho)$ belongs to $V$ (since $\left.\rho^{\star}>0\right)$. Then, thanks to the previous estimates, one has $(u, \rho) \in W$. This proves that Hypothesis 2 of Theorem A.1 is satisfied. We may apply Theorem A.1 and this concludes the proof of Proposition A.1.

\section{B Error estimates for a class of staggered schemes}

In this section we present some alternative numerical schemes for the approximation of problem (1.1)(1.5), called staggered schemes. It is intended to provide basic notions for these schemes in order to be able to state the main result. It is not intend to be self contained.

The space discretization in these schemes is staggered using nonconforming low-order finite element approximations, namely the Rannacher and Turek element (RT) [39] for quadrilateral or hexahedric meshes, or the lowest degree Crouzeix-Raviart element (CR) [7] for simplicial meshes. We invite the reader wishing to read more about the discretizations of compressible flows via the staggered schemes to consult [21], [12], [20], [26].

By the approach presented in this paper, it is possible to establish for these schemes similar error estimates as those established in Theorem 3.2 for the MAC scheme. The exact result is stated in Theorem B.1. This is possible due to the fact that the structure of staggered schemes and the MAC scheme present many common features as was shown in [30], where the authors have developed a uniform formalism for all above mentioned staggered schemes. To prove Theorem B.1, it is therefore enough to follow for these discretizations the same process whose great lines are described after Remark 2, bringing to light the same leading terms both in the relative energy inequality and in the consistency error estimates. This is the main idea beyond the proof of Theorem B.1. Its realization, however, remains still laborious.

\section{B.1 Space and time discretization}

From now, let $\Omega \subset \mathbb{R}^{3}$ be a bounded polyhedral domain. Let $\mathscr{M}$ be a decomposition of the domain $\Omega$ in non-degenerate quadrilaterals $(\mathrm{d}=2)$ or hexahedra $(\mathrm{d}=3)$ or simplices, both type of cells being possibly combined in a same mesh. By $\mathscr{E}(K)$, we denote the set of the edges $(\mathrm{d}=2)$ or faces $(\mathrm{d}=3) \sigma$ of the element $K \in \mathscr{M}$; for short, each edge or face will be called an edge hereafter. The set of all edges of the mesh is denoted by $\mathscr{E}$; the set of edges included in the boundary of $\Omega$ is denoted by $\mathscr{E}_{\text {ext }}$ and the set of internal edges (i.e $\mathscr{E} \backslash \mathscr{E}_{\text {ext }}$ ) is denoted by $\mathscr{E}_{\text {int }}$. The decomposition $\mathscr{M}$ verifies the following assumption: $\bar{\Omega}=\cup_{K \in \mathscr{M}} \bar{K}$; if $K, L \in \mathscr{M}$, then $\bar{K} \cap \bar{L}=\emptyset, \bar{K} \cap \bar{L}$ is a vertex or $\bar{K} \cap \bar{L}$ is a common edge of $K$ and $L$, which is denoted by $K \mid L$. For each internal edge of the mesh $\sigma=K \mid L, \boldsymbol{n}_{K L}$ stands for the normal vector of $\sigma$, oriented form $K$ to $L$ (so that $\left.\boldsymbol{n}_{K L}=-\boldsymbol{n}_{L K}\right)$. By $|K|$ and $|\sigma|$ we denote the $(d$ and $d-1$ dimensional) measure, respectively, of an element $K$ and of an edge $\sigma$, and $h_{K}$ and $h_{\sigma}$ stand for the diameter of $K$ and $\sigma$, respectively. As in the MAC case, we measure the size of the mesh through the parameter $h_{\mathscr{M}}$ defined by

$$
h_{\mathscr{M}}=\max \left\{h_{K}, K \in \mathscr{M}\right\},
$$


where $h_{K}$ stands for the diameter of $K$. We measure the regularity of the mesh through the parameter $\theta_{\mathscr{M}}$ defined by

$$
\theta_{\mathscr{M}}=\min \left\{\frac{\xi_{K}}{h_{K}}, K \in \mathscr{M}\right\}
$$

where $\xi_{K}$ stands for the diameter of the largest ball included in $K$.

Let us briefly describe the Crouzeix-Raviart element for simplicial meshes (see [7] for the seminal paper and, for instance, [9, p. 83-85], for a synthetic presentation), and the so-called 'rotated bilinear element' introduced by Rannacher and Turek for quadrilateral or hexahedric meshes [39]. The reference element for the Crouzeix-Raviart element is the unit d-simplex and the discrete function space is the space $\mathbb{P}_{1}$ of affine polynomials. The reference element $\hat{K}$ for the rotated bilinear element is the unit $d$-cube (with edges parallel to the coordinate axes); the discrete function space on $\hat{K}$ is $\tilde{\mathbb{Q}}_{1}(\hat{K})$, where $\tilde{\mathbb{Q}}_{1}(\hat{K})$ is defined as follows

$$
\tilde{\mathbb{Q}}_{1}(\hat{K})=\operatorname{span}\left\{1,\left(x_{i}\right)_{i=1, \ldots, d},\left(x_{i}^{2}-x_{i+1}^{2}\right)_{i=1, \ldots, d-1}\right\} .
$$

For both velocity elements used here, the degrees of freedom are determined by the following set of nodal functionals:

$$
\left.\left\{m_{\sigma, i}, \sigma \in \mathscr{E}(K), i=1, \ldots d\right\}, \quad m_{\sigma, i}(\boldsymbol{v})=\frac{1}{|\sigma|} \int_{\sigma} v_{i} \mathrm{~d} \boldsymbol{x}, \boldsymbol{v}=\left(v_{1}, \ldots, v_{d}\right)\right\} .
$$

The mapping from the reference element to the actual one is, for the Rannacher-Turek element, the standard $Q_{1}$ mapping and, for the Crouzeix-Raviart element, the standard affine mapping. Finally, in both cases, the continuity of the average value of discrete velocities (i.e., for a discrete velocity field $\boldsymbol{v}$, $\left.m_{\sigma, i}(\boldsymbol{v}), 1 \leq i \leq d\right)$ across each edge of the mesh is required, thus the discrete space $\boldsymbol{W}_{\mathscr{E}, 0}(\Omega)$ is defined as follows:

$$
\begin{aligned}
\boldsymbol{W}_{\mathscr{E}, 0}(\Omega)=\left[W_{\mathscr{E}, 0}(\Omega)\right]^{d}=\left\{\boldsymbol{v} \in L^{2}(\Omega)^{d}, \forall K \in \mathscr{M}, \boldsymbol{v}_{\mid K} \in W(K)^{d} \text { and } \forall \sigma=K \mid L \in \mathscr{E}_{\text {int }},\right. \\
\left.m_{\sigma, i}\left(\boldsymbol{v}_{\mid K}\right)=m_{\sigma, i}\left(\boldsymbol{v}_{\mid L}\right), \forall \sigma \in \mathscr{E}_{\text {ext }}, m_{\sigma, i}(\boldsymbol{v})=0\right\}
\end{aligned}
$$

where $W(K)$ is the space of functions on $\mathrm{K}$ generated by $\tilde{\mathbb{Q}}_{1}(\hat{K})$ through the $Q_{1}$ mapping from $\hat{K}$ to $K$ for the Rannacher-Turek element and the space of affine functions on $K$ for the Crouzeix-Raviart element.

From the definition (B.3), each velocity degree of freedom can be uniquely associated to an element edge. More precisely the degrees of freedom for the velocity components are located at the center of the faces of the mesh. Hence, the velocity degrees of freedom may be indexed by the number of the component and the associated edge, and the set of velocity degrees of freedom reads:

$$
\left\{\boldsymbol{u}_{\sigma}, \sigma \in \mathscr{E}\right\} .
$$

Finally, we need to deal with the Dirichlet boundary condition. Since the velocity unknowns lie on the boundary (and not inside the cells), these conditions are taken into account in the definition of the discrete spaces by setting zero to the velocity unknows that lie on the boundary

$$
\forall \sigma \in \mathscr{E}_{\text {ext }}, \quad \boldsymbol{u}_{\sigma}=0
$$

Since only the continuity of the integral over each edge of the mesh is imposed, the functions of $\boldsymbol{W}_{\mathscr{E}, 0}(\Omega)$ are discontinous through each edge; the discretization is thus nonconforming in $H^{1}(\Omega)^{d}$.

We denote by $\varphi_{\sigma}$ the function of $W_{\mathscr{E}, 0}(\Omega)$ such that

$$
\int_{\sigma^{\prime}} \varphi_{\sigma} \mathrm{d} \gamma=\left|\sigma^{\prime}\right| \delta_{\sigma, \sigma^{\prime}} \text { for any } \sigma, \sigma^{\prime} \in \mathscr{E}_{\text {int }} .
$$

The degrees of freedom for the density (i.e. the discrete density unknowns) are associated to the cells of the mesh $\mathscr{M}$, and are denoted by:

$$
\left\{\varrho_{K}, K \in \mathscr{M}\right\} .
$$


We now introduce a dual mesh, which will be used for the finite volume approximation of the time derivative and the convective terms in the momentum balance equation. In contrast with the MAC scheme, the dual mesh is the same for all velocity components. When $K \in \mathscr{M}$ is a simplex, a rectangle or a cuboid, for $\sigma \in \mathscr{E}(K)$, we define $D_{\sigma, K}$ as the cone with basis $\sigma$ and with vertex the mass center of $K$. We thus obtain a partition of $K$ in $m$ sub-volumes, where $m$ is the number of faces of the mesh, each sub-volume having the same measure $\left|D_{\sigma, K}\right|=|K| / m$. We extend this definition to general quadrangles and hexahedra, by supposing that we have built a partition still of equal-volume sub-cells, and with the same connectivities. Note that this is of course always possible, but that such a volume $D_{\sigma, K}$ may be no longer a cone; indeed, if $K$ is far from a parallelogram, it may not be possible to build a cone having $\sigma$ as basis, the opposite vertex lying in $K$ and a volume equal to $|K| / m$. The volume $D_{\sigma, K}$ is referred to as the half-diamond cell associated to $K$ and $\sigma$.

For $\sigma \in \mathscr{E}_{\text {int }}, \sigma=K \mid L$, we now define the diamond cell $D_{\sigma}$ associated to $\sigma$ by $D_{\sigma}=D_{\sigma, K} \cup D_{\sigma, L}$; for an external face $\sigma \in \mathscr{E}_{\text {ext }} \cap \mathscr{E}(K), D_{\sigma}$ is just the same volume as $D_{\sigma, K}$. We define the space $\boldsymbol{S}_{\mathscr{E}}(\Omega)$ of vector valued functions constant on every $D_{\sigma}, \sigma \in \mathscr{E}$. We denote by $\boldsymbol{S}_{\mathscr{E}, 0}(\Omega)$ the subspace of functions from $\boldsymbol{S}_{\mathscr{E}}(\Omega)$ that are zero on every $D_{\sigma}, \sigma \in \mathscr{E}_{\text {ext }}$. We then introduce the following operator

$$
\mathscr{P}_{\mathscr{E}}: \mid \begin{aligned}
& \boldsymbol{W}_{\mathscr{E}, 0}(\Omega) \longrightarrow \boldsymbol{S}_{\mathscr{E}, 0}(\Omega) \\
& \boldsymbol{u} \longmapsto \mathscr{P}_{\mathscr{E}} \boldsymbol{u}=\sum_{\sigma \in \mathscr{E}_{\text {int }}} \boldsymbol{u}_{\sigma} \mathscr{X}_{D_{\sigma}}(\boldsymbol{x}),
\end{aligned}
$$

which is clearly a one to one mapping.

The density on a dual cell is given by:

$$
\begin{array}{ll}
\text { for } \sigma \in \mathscr{E}_{\text {int }}, \sigma=K \mid L & \left|D_{\sigma}\right| \varrho_{D_{\sigma}}=\left|D_{\sigma, K}\right| \varrho_{K}+\left|D_{\sigma, L}\right| \varrho_{L}, \\
\text { for } \sigma \in \mathscr{E}_{\text {ext }}, \sigma \in \mathscr{E}(K), \quad & \varrho_{D_{\sigma}}=\varrho_{K} .
\end{array}
$$

and we denote

$$
\hat{\varrho}=\sum_{\sigma \in \mathscr{E}} \varrho_{D_{\sigma}} \mathscr{X}_{D_{\sigma}}(\boldsymbol{x})
$$

For the the time discretization of problem (1.1)-(1.5), we consider a partition $0=t^{0}<t^{1}<\cdots<$ $t^{N}=T$ of the time interval $(0, T)$, and, for the sake of simplicity, a constant time step $\delta t=t^{n}-t^{n-1}$; hence $t^{n}=n \delta t$ for $n \in\{0, \cdots, N\}$. We denote respectively by $\left\{\boldsymbol{u}_{\sigma}^{n}, \sigma \in \mathscr{E}_{\text {int }}, n \in\{0, \cdots, N\}\right\}$, and $\left\{\varrho_{K}^{n}, K \in \mathscr{M}, n \in\{1, \cdots, N\}\right)$ the sets of discrete velocity and density unknowns. For $\sigma \in \mathscr{E}_{\text {int }}$, the value $\boldsymbol{u}_{\sigma}^{n}$ is an expected approximation of the mean value over $\left(t^{n-1}, t^{n}\right) \times D_{\sigma}$ of the velocity of a weak solution, while for $K \in \mathscr{M}$ the value $\varrho_{K}^{n}$ is an expected approximation of the mean value over $\left(t^{n-1}, t^{n}\right) \times K$ of the density of a weak solution. To the discrete unknowns, we associate piecewise constant functions on time intervals and on primal or dual meshes, which are expected approximation of weak solutions, For the velocity, this constant function is of the form:

$$
\boldsymbol{u}(t, \boldsymbol{x})=\sum_{n=1}^{N} \sum_{\sigma \in \mathscr{E}_{\mathrm{int}}} \boldsymbol{u}_{\sigma}^{n} \mathscr{X}_{D_{\sigma}}(\boldsymbol{x}) \mathscr{X}_{\left(t^{n-1}, t^{n}\right)}(t),
$$

where $\mathscr{X}_{\left(t^{n-1}, t^{n}\right)}$ is the characteristic function of the interval $\left(t^{n-1}, t^{n}\right)$. We denote by $\boldsymbol{X}_{\mathscr{E}, \delta t}$ the set of such piecewise constant functions on time intervals and dual cells. For the density, the constant function is of the form:

$$
\varrho(t, \boldsymbol{x})=\varrho_{K}^{n} \text { for } \boldsymbol{x} \in K \text { and } t \in\left(t^{n-1}, t^{n}\right),
$$

and we denote by $Y_{\mathscr{M}, \delta t}$ the space of such piecewise constant functions.

For a given $\boldsymbol{u} \in \boldsymbol{X}_{\mathscr{E}, \delta t}$ associated to the set of discrete velocity unknowns $\left\{\boldsymbol{u}_{\sigma}^{n}, \sigma \in \mathscr{E}_{\text {int }}, n \in\right.$ $\{1, \cdots, N\}\}$, and for $n \in\{1, \cdots, N\}$, we denote by $\boldsymbol{u}^{n} \in \boldsymbol{S}_{\mathscr{E}, 0}(\Omega)$ the piecewise constant function defined by $\boldsymbol{u}^{n}(\boldsymbol{x})=\boldsymbol{u}_{\sigma}^{n}$ for $\boldsymbol{x} \in D_{\sigma}, \sigma \in \mathscr{E}_{\text {int }}$. In a same way, given $\varrho \in Y_{\mathscr{M}, \delta t}$ associated to the discrete density unknows $\left\{\varrho_{K}^{n}, K \in \mathscr{M}, n \in\{1, \cdots, N\}\right\}$ we denote by $\varrho^{n} \in L_{\mathscr{M}}$ the piecewise constant function defined by $\varrho^{n}(\boldsymbol{x})=\varrho_{K}^{n}$ for $\boldsymbol{x} \in K, K \in \mathscr{M}$. 
We consider an implicit-in-time scheme, which reads in its fully discrete form, for $1 \leq n \leq N$ and $1 \leq i \leq d:$

$$
\begin{aligned}
& \frac{1}{\delta t}\left(\varrho^{n}-\varrho^{n-1}\right)+\operatorname{div}_{\mathscr{M}}^{\mathrm{up}}\left(\varrho^{n} \boldsymbol{u}^{n}\right)=0, \\
& \begin{array}{r}
\frac{1}{\delta t}\left(\varrho^{n} \boldsymbol{u}^{n}-\widehat{\varrho^{n-1}} \boldsymbol{u}^{n-1}\right)+\operatorname{div}_{\mathscr{E}}\left(\varrho^{n} \boldsymbol{u}^{n} \otimes \boldsymbol{u}^{n}\right)-\mu \Delta_{\mathscr{E}} \boldsymbol{u}^{n} \\
\quad-(\mu+\lambda) \nabla_{\mathscr{E}} \operatorname{div} \mathscr{M} \boldsymbol{u}^{n}+\nabla_{\mathscr{E}} p\left(\varrho^{n}\right)=0,
\end{array}
\end{aligned}
$$

where the terms introduced for each discrete equation are defined hereafter.

\section{B.1.1 Mass balance equation}

As for the MAC scheme, equation (B.8a) is a finite volume discretization of the mass balance (1.1a) over the primal mesh. The discrete "upwind" divergence is defined by

$$
\begin{array}{l|l}
\operatorname{div}_{\mathscr{M}}^{\text {up }}: & S_{\mathscr{M}}(\Omega) \times \boldsymbol{S}_{\mathscr{E}, 0}(\Omega) \longrightarrow S_{\mathscr{M}}(\Omega) \\
& (\varrho, \boldsymbol{u}) \longmapsto \operatorname{div}_{\mathscr{M}}^{\mathrm{up}}(\varrho \boldsymbol{u})=\sum_{K \in \mathscr{M}} \frac{1}{|K|} \sum_{\sigma \in \mathscr{E}(K)} F_{\sigma, K}(\varrho, \boldsymbol{u}) \mathscr{X}_{K},
\end{array}
$$

where $F_{\sigma, K}(\varrho, \boldsymbol{u})$ stands for the mass flux across $\sigma$ outward $K$, which, because of the Dirichlet boundary conditions, vanishes on external faces and is given on the internal faces by:

$$
\forall \sigma=K\left|L \in \mathscr{E}_{\text {int }}, \quad F_{\sigma, K}(\varrho, \boldsymbol{u})=\right| \sigma \mid \varrho_{\sigma}^{\text {up }} u_{\sigma, K},
$$

where $u_{\sigma, K}$ is an approximation of the normal velocity to the face $\sigma$ outward $K$, defined by:

$$
u_{\sigma, K}=\boldsymbol{u}_{\sigma} \cdot \boldsymbol{n}_{\sigma, K} \text { for } \sigma \in \mathscr{E}(K) .
$$

Thanks to the boundary conditions, $u_{\sigma, K}$ vanishes for any external face $\sigma$. The density at the internal face $\sigma=K \mid L$ is obtained by an upwind technique:

$$
\varrho_{\sigma}^{\text {up }}=\mid \begin{array}{ll}
\varrho_{K} & \text { if } u_{\sigma, K} \geq 0 \\
\varrho_{L} & \text { otherwise }
\end{array}
$$

\section{B.1.2 The momentum equation}

We now turn to the discrete momentum balances (B.8b), which are obtained by discretizing the momentum balance equation (1.1b) on the dual cells associated to the faces of the mesh.

The discrete convective operator - The discrete divergence of the convective term $\varrho u \otimes u$ is defined by

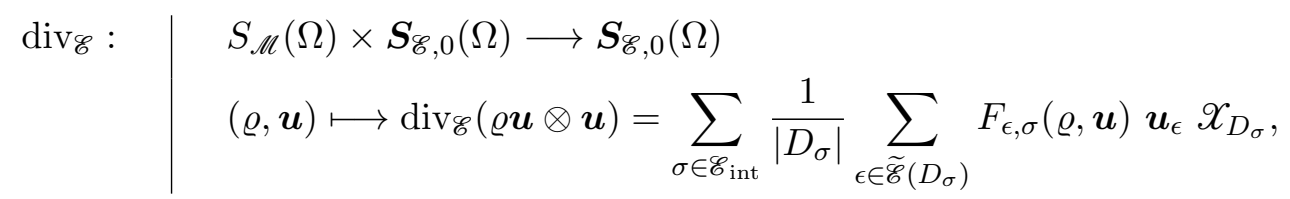

where for $\sigma \in \mathscr{E}_{\text {int }}$ and $\epsilon \in \mathscr{E}\left(D_{\sigma}\right)$ the quantity $F_{\epsilon, \sigma}=F_{\epsilon, \sigma}(\varrho, \boldsymbol{u})$ stands for a mass flux through the dual faces of the mesh and is defined hereafter while $u_{\epsilon}$ stands for an approximation of $i^{t h}$ component of the velocity over $\epsilon$ in the case of $\sigma \in \mathscr{E}^{(i)}$. First of all by virtue of the Dirichlet boundary condition, that the flux through a dual face included in the boundary is taken equal to zero. For $K \in \mathscr{M}$ and $\sigma \in \mathscr{E}(K)$, let $\xi_{K}^{\sigma}$ be given by:

$$
\xi_{K}^{\sigma}=\frac{\left|D_{\sigma, K}\right|}{|K|} .
$$

With the definition of the dual mesh adopted here, the value of the coefficients $\xi_{K}^{\sigma}$ is independent of the cell and the face. For the Rannacher-Turek elements, we have $\xi_{K}^{\sigma}=1 /(2 d)$ and, for the Crouzeix-Raviart elements, $\xi_{K}^{\sigma}=1 /(d+1)$. We suppose first that the flux through the external dual faces, which are also faces of the primal mesh, is equal to zero.

Then the mass fluxes through the inner dual faces are supposed to satisfy the following properties. 
Definition B.1 (Definition of the dual fluxes from the primal ones). The fluxes through the faces of the dual mesh are defined so as to satisfy the following three constraints:

(H1) The discrete mass balance over the half-diamond cells is satisfied, in the following sense. For all primal cell $K$ in $\mathscr{M}$, the set $\left(F_{\epsilon, \sigma}\right)_{\epsilon \subset K}$ of dual fluxes included in $K$ solves the following linear system

$$
F_{\sigma, K}+\sum_{\epsilon \in \widetilde{\mathscr{E}}\left(D_{\sigma}\right), \epsilon \subset K} F_{\epsilon, \sigma}=\xi_{K}^{\sigma} \sum_{\sigma^{\prime} \in \mathscr{E}(K)} F_{\sigma^{\prime}, K}, \quad \sigma \in \mathscr{E}(K) .
$$

(H2) The dual fluxes are conservative, i.e. for any dual face $\epsilon=D_{\sigma} \mid D_{\sigma}^{\prime}$, we have $F_{\epsilon, \sigma}=-F_{\epsilon, \sigma^{\prime}}$.

(H3) The dual fluxes are bounded with respect to the primal fluxes $\left(F_{\sigma, K}\right)_{\sigma \in \mathscr{E}(K)}$, in the sense that there exists a constant real number $C$ such that:

$$
\left|F_{\epsilon, \sigma}\right| \leq C \max \left\{\left|F_{\sigma, K}\right|, \sigma \in \mathscr{E}(K)\right\}, \quad K \in \mathscr{M}, \sigma \in \mathscr{E}(K), \epsilon \in \widetilde{\mathscr{E}}\left(D_{\sigma}\right), \epsilon \subset K .
$$

In fact, definition B.1 is not complete, since the system of equations (B.15) has an infinite number of solutions, which makes necessary to impose in addition the constraint (B.16); however, assumptions (H1)-(H3) are sufficient for the subsequent developments of this paper. A detailed process of the dual fluxes construction can be found in [1,25].

Since the flux across a dual face lying on the boundary is zero, the values $u_{\epsilon}$ are only needed at the internal dual faces, and we make the centered choice for their discretization, i.e. for $\epsilon=D_{\sigma} \mid D_{\sigma^{\prime}} \in \tilde{\mathscr{E}}_{\text {int }}$,

$$
\boldsymbol{u}_{\epsilon}=\frac{\boldsymbol{u}_{\sigma}+\boldsymbol{u}_{\sigma^{\prime}}}{2}
$$

The discrete divergence and gradient - The discrete divergence $\operatorname{div} \mathscr{M} \in \mathscr{L}\left(\boldsymbol{S}_{\mathscr{C}, 0}(\Omega), S_{\mathscr{M}}(\Omega)\right)$ of the velocity (or more generally of a function $\boldsymbol{S}_{\mathscr{E}, 0}(\Omega)$ ) has a natural approximation:

$$
\text { for } K \in \mathscr{M}, \quad\left(\operatorname{div}_{\mathscr{M}} \boldsymbol{u}\right)_{K}=\frac{1}{|K|} \sum_{\sigma \in \mathscr{E}(K)}|\sigma| u_{\sigma, K}
$$

The term $\left(\nabla_{\mathscr{E}} p\right)_{\sigma}$ stands for the discrete pressure gradient at the face $\sigma$. This gradient operator, which belongs to $\mathscr{L}\left(S_{\mathscr{M}}(\Omega), \boldsymbol{S}_{\mathscr{E}, 0}(\Omega)\right)$ is built as the transpose of the discrete operator for the divergence of the velocity, i.e. in such a way that the following duality relation with respect to the $\mathrm{L}^{2}$ inner product holds:

$$
\sum_{K \in \mathscr{M}}|K| p_{K}\left(\operatorname{div}_{\mathscr{M}} \boldsymbol{u}\right)_{K}+\sum_{\sigma \in \mathscr{E}_{\text {int }}}\left|D_{\sigma}\right| \boldsymbol{u}_{\sigma} \cdot\left(\nabla_{\mathscr{E}} p\right)_{\sigma}=0
$$

This yields to the following expression:

$$
\text { for } \sigma=K \mid L \in \mathscr{E}_{\text {int }}, \quad\left(\nabla_{\mathscr{E}} p\right)_{\sigma}=\frac{|\sigma|}{\left|D_{\sigma}\right|}\left(p_{L}-p_{K}\right) \boldsymbol{n}_{\sigma, K}
$$

Note that, because of the Dirichlet boundary conditions, the discrete gradient is not defined at the external faces.

Discrete Laplace operator - The discrete Laplace operator $\Delta_{\mathscr{C}} \in \mathscr{L}\left(\boldsymbol{S}_{\mathscr{E}, 0}(\Omega), \boldsymbol{S}_{\mathscr{\delta}, 0}(\Omega)\right)$ reads for $\boldsymbol{u} \in \boldsymbol{S}_{\mathscr{E}, 0}(\Omega)$ and $\sigma \in \mathscr{E}_{\text {int }}$ :

$$
\left(-\Delta_{\mathscr{E}} \boldsymbol{u}\right)_{\sigma}=\int_{\Omega} \nabla_{\mathscr{M}} \mathscr{P}_{\mathscr{E}}^{-1} \boldsymbol{u}: \nabla_{\mathscr{M}} \boldsymbol{\varphi}_{\sigma} \mathrm{d} \boldsymbol{x},
$$

where $\boldsymbol{\varphi}_{\sigma}=\left(\varphi_{\sigma}, \ldots, \varphi_{\sigma}\right) \in \boldsymbol{W}_{\mathscr{E}, 0}(\Omega)$, where the shape function $\varphi_{\sigma}$ is introduced in $(B .5)$ and where $\mathscr{P}_{\mathscr{C}}$ is defined in (B.6). In the above formula and for a function $\boldsymbol{v} \in \boldsymbol{W}_{\mathscr{E}, 0}(\Omega)$, the quantity $\nabla \boldsymbol{v}$ is equal to the gradient of the function $\boldsymbol{v}$ almost everywhere in $\Omega$.

Here again let us introduce the discrete relative energy functional

$$
\mathscr{E}\left(\varrho^{n}, \boldsymbol{u}^{n} \mid r_{\mathscr{M}}^{n}, \boldsymbol{U}_{\mathscr{E}}^{n}\right)=\sum_{\sigma \in \mathscr{E}_{\text {int }}} \frac{1}{2}\left|D_{\sigma}\right| \varrho_{D_{\sigma}}^{n}\left|\boldsymbol{u}_{\sigma}^{n}-\boldsymbol{U}_{\sigma}^{n}\right|^{2}+\sum_{K \in \mathscr{M}}|K| E\left(\varrho_{K}^{n} \mid r_{K}^{n}\right)
$$

where

$$
r^{n}=r\left(t^{n}, \cdot\right), \boldsymbol{U}^{n}=\boldsymbol{U}\left(t^{n}, \cdot\right), r_{K}^{n}=\frac{1}{|K|} \int_{K} r^{n} \mathrm{~d} \boldsymbol{x}, \boldsymbol{U}_{\sigma}^{n}=\frac{1}{|\sigma|} \int_{\sigma} \boldsymbol{U}^{n} \mathrm{~d} \gamma
$$

Now, we are ready to state the result about the error estimate for these alternative discretizations. 
Theorem B.1 (Error estimate). Let $\Omega \subset \mathbb{R}^{3}$ be a bounded polyhedral domain. Assume that the viscosity coefficients satisfy assumptions (1.4) and that the pressure $p$ satisfy (1.5) with $\gamma>3 / 2$. Let $\mathscr{M}$ be a decomposition of the domain $\Omega$ in simplices, with step size $h_{\mathscr{M}}$ (see (B.1)) and regularity $\theta_{\mathscr{M}}$ where $\theta_{\mathscr{M}}$ is defined in (B.2). Let us consider a partition $0=t^{0}<t^{1}<\ldots<t^{N}=T$ of the time interval $[0, T]$, which, for the sake of simplicity, we suppose uniform where $\delta t$ stands for the constant time step. Let $(\varrho, \boldsymbol{u}) \in$ $Y_{\mathscr{M}, \delta t} \times \boldsymbol{X}_{\mathscr{E}, \delta t}$ be a solution of the discrete problem (B.8) emanating from $\left(\varrho^{0}, \boldsymbol{u}^{0}\right) \in S_{\mathscr{M}}(\Omega) \times \boldsymbol{S}_{\mathscr{E}, 0}(\Omega)$ such that $\varrho^{0}>0$ and $(r, \boldsymbol{U}) \in \mathscr{F}$ (see (3.73)) be a (strong) solution of problem (1.1)-(1.5). Then there exists a constant $c>0$ only depending on $T, \Omega, p_{0}, p_{\infty}, \mu, \gamma, \alpha, \underline{r}, \min _{[\underline{r}, \bar{r}]} p, \min _{[\underline{r} / 2,2 \bar{r}]} p^{\prime}$, on $\|(r, \boldsymbol{U})\|_{\mathscr{F}}$, on $\mathscr{E}_{0, \mathscr{M}}$ in a nondecreasing way and on $\theta_{\mathscr{M}}$ in a nonincreasing way such that

$$
\max _{0 \leq n \leq N} \mathscr{E}\left(\varrho^{n}, \boldsymbol{u}^{n} \mid r_{\mathscr{M}}^{n}, \boldsymbol{U}_{\mathscr{E}}^{n}\right) \leq c\left(\mathscr{E}\left(\varrho^{0}, \boldsymbol{u}^{0} \mid r_{\mathscr{M}}^{0}, \boldsymbol{U}_{\mathscr{C}}^{0}\right)+h_{\mathscr{M}}^{A}+\sqrt{\delta t}\right),
$$

where $A$ is given by

$$
A=\min \left(\frac{2 \gamma-3}{\gamma}, \frac{1}{2}\right)
$$

Remark 4. 1. The discrete problem (B.8) admits a solution. As for the MAC case, the proof is based on a topological degree argument.

2. Note that the exponent $A$ is the same for all discretizations investigated in this paper.

3. The items listed in Remark 2 remain valid also for discretizations described in this section.

\section{References}

[1] G. Ansanay-Alex, F. Babik, J-C. Latché, and D. Vola. An L²-stable approximation of the NavierStokes convection operator for low-order non-conforming finite elements. International Journal for Numerical Methods in Fluids., 66:555-580, 2011.

[2] H. Bijl and P. Wesseling. A unified method for computing incompressible and compressible flows in boundary-fitted coordinates. J. Comp. Phys., 141:153-173, 1998.

[3] CALIF3S. A software components library for the computation of reactive turbulent flows. https: //gforge.irsn.fr/gf/project/calif3s

[4] C. Cancès, H. Mathis, and N. Seguin. Relative entropy for the finite volume approximation of strong solutions to systems of conservation laws. SIAM J. Numer. Anal., 54(2):263-1287, 2016.

[5] V. Casulli and D. Greenspan. Pressure method for the numerical solution of transient, compressible fluid flows. Intern. J. Numer. Method. Fluid., 4:1001-1012, 1984.

[6] P. Colella and K. Pao. A projection method for low speed flows. J. Comp. Phys., 149:245-269, 1999.

[7] P. Colella and K. Pao. Conforming and nonconforming finite element methods for solving the stationary Stokes equations. Revue française d'automatique, informatique, recherche opérationnelle. Mathématique., 7(3):33-75, 1973.

[8] K. Deimling. Nonlinear functional analysis. Springer-Verlag, Berlin, 1985.

[9] A. Ern and J-L. Guermond. Eléments finis: théorie, applications, mise en oeuvre. Springer Science, Business Media, 36, 2002.

[10] R. Eymard, T. Gallouët, M. Ghilani, and R. Herbin. Error estimates for the approximate solutions of a nonlinear hyperbolic equation given by finite volume schemes. IMA J. Numer. Anal., 18(4):563594, 1998.

[11] R. Eymard, T. Gallouët, and R. Herbin. Finite volume methods. Handbook of numerical analysis, 7:713-1018. North-Holland, Amsterdam, 2000. 
[12] R. Eymard, T. Gallouët, R. Herbin J-C. Latché. A convergent finite element-finite volume scheme for the compressible Stokes problem. II. The isentropic case. Math. Comp., 270(79):649-675, 2010.

[13] E. Feireisl. Dynamics of viscous compressible fluids, volume 26 of Oxford Lecture Series in Mathematics and its Applications. Oxford University Press, Oxford, 2004.

[14] E. Feireisl, R. Hošek, D. Maltese, and A. Novotný. Error estimates for a numerical method for the compressible Navier-Stokes system on sufficiently smooth domains. ESAIM : Mathematical Modelling and Numerical Analysis, 51(1):279-319, 2017.

[15] E. Feireisl, B J. Jin, and A. Novotný. Relative entropies, suitable weak solutions, and weak-strong uniqueness for the compressible Navier-Stokes system. J. Math. Fluid. Mech., 14(4):717-730, 2012.

[16] E. Feireisl and M. Lukačová-Medvidová. Convergence of a mixed finite element finite volume scheme for the isentropic Navier-Stokes system via dissipative measure-valued solutions. Foundations of Computational Mathematics, 18(3):703-730, 2018.

[17] E. Feireisl, A. Novotný, and H. Petzeltová. On the existence of globally defined weak solutions to the Navier - Stokes equations. Journal of Mathematical Fluid Mechanics, 3(4):358-392, 2001.

[18] E. Feireisl, A. Novotný. Weak-strong uniqueness property for the full Navier-Stokes-Fourier system Archive for Rational Mechanics and Analysis, 204(2), 683-706, 2012.

[19] E. Feireisl, A. Novotný, and Y. Sun. Suitable weak solutions to the navier-stokes equations of compressible viscous fluids. Indiana Univ. Math. J, 60(2):611-631, 2011.

[20] A. Fettah and T. Gallouët. Numerical approximation of the general compressible Stokes problem. IMA J. Numer. Anal., 33(3):922-951, 2013.

[21] T. Gallouët, L. Gastaldo, J-C. Latché, and R. Herbin. An unconditionally stable pressure correction scheme for compressible barotropic Navier-Stokes equations. M2AN Math. Model. Numer. Anal. $44(2): 251-287,2010$.

[22] T. Gallouët, J-C. Latché, and R. Herbin. $W^{1, q}$ stability of the Fortin operator for the MAC scheme. Calcolo 49(1):63-71, 2012.

[23] T. Gallouët, R. Herbin, J-C Latché, and K Mallem. Convergence of the MAC scheme for the incompressible Navier-Stokes equations. Foundations of Computational Mathematics, 18(1): 249$289,2018$.

[24] T. Gallouët, R. Herbin, D. Maltese, and A. Novotny. Error estimates for a numerical approximation to the compressible barotropic Navier-Stokes equations. IMA J. Numer. Anal., 36(2):543-592, 2016.

[25] L. Gastaldo, R. Herbin, W. Kheriji, C. Lapuerta, and J-C. Latché. Staggered discretizations, pressure correction schemes and all speed barotropic flows. Finite Volumes for Complex Applications VI Problems and Perspectives - Prague, Czech Republic, 2:39-56, 2011.

[26] L. Gastaldo, R. Herbin, J-C. Latché, and N. Therme. Consistent explicit staggered schemes with muscle and artificial viscosity techniques for the Euler equations. Personnal communication, 2015.

[27] F.H. Harlow and A.A. Amsden. Numerical calculation of almost incompressible flow. J. Comp. Phys., 3:80-93, 1968.

[28] F.H. Harlow and A.A. Amsden. A numerical fluid dynamics calculation method for all flow speeds. J. Comp. Phys., 8:197-213, 1971.

[29] F.H Harlow, J E Welch, and al. Numerical calculation of time-dependent viscous incompressible flow of fluid with free surface. Phys. Fluid., 8(12):21-82, 1965.

[30] R. Herbin, J-C. Latché, and N. Therme. Consistency results of a class of staggered schemes for the compressible Euler equations. Article in preparation, Chapter 3 of the PhD thesis of N. Therme, https://old.i2m.univ-amu.fr/ nicolas.therme/Manuscrit_These.pdf 
[31] R. Hošek and B. She. Stability and consistency of a finite difference scheme for compressible viscous isentropic flow in multi-dimension. Journal of Numerical Mathematics, 26(3):111-140, 2018.

[32] R.I. Issa. Solution of the implicitly discretised fluid flow equations by operator splitting. J. Comp. Phys., 62:40-65, 1985.

[33] R.I. Issa, A.D. Gosman, and A.P. Watkins. The computation of compressible and incompressible recirculating flows by a non-iterative implicit scheme. J. Comp. Phys., 62:66-82, 1986.

[34] V. Jovanović and C. Rohde. Finite-volume schemes for friedrichs systems in multiple space dimensions: A priori and a posteriori error estimates. Numerical Methods for Partial Differential Equations, 21(1):104-131, 2005.

[35] K.C. Karki and S.V. Patankar. Pressure based calculation procedure for viscous flows at all speeds in arbitrary configurations. AIAA J., 27:1167-1174, 1989.

[36] T K. Karper. A convergent FEM-DG method for the compressible Navier-Stokes equations. Numer. Math., 125(3) : 441-510, 2013.

[37] P-L. Lions. Mathematical topics in fluid mechanics. Vol. 2, volume 10 of Oxford Lecture Series in Mathematics and its Applications. Oxford University Press, New York, 1998.

[38] A. Novotný and I. Straskraba. Introduction to the mathematical theory of compressible flow. volume 27 of Oxford Lecture Series in Mathematics and its Applications. Oxford University Press, New York, 2004.

[39] R. Rannacher and S. Turek. Simple nonconforming quadrilateral Stokes element. Numerical Methods for Partial Differential Equations, 8(2):97-111, 1992.

[40] A. Valli and W. M. Zajaczkowski. Navier-Stokes equations for compressible fluids: global existence and qualitative properties of the solutions in the general case. Communications in Mathematical Physics, 103(2):259-296, 1986.

[41] D.R. van der Heul, C. Vuik, and P. Wesseling. Stability analysis of segregated solution methods for compressible flow. App. Numer. Math., 38:257-274, 2001.

[42] D.R. van der Heul, C. Vuik, and P. Wesseling. A conservative pressure-correction method for flow at all speeds. Comp. Fluid., 32:1113-1132, 2003.

[43] D. Vidović, A. Segal, and P. Wesseling. A superlinearly convergent Mach-uniform finite volume method for the Euler equations on staggered unstructured grids. J. Comp. Phys., 217:277-294, 2006 .

[44] J-P. Vila and P. Villedieu. Convergence of an explicit finite volume scheme for first order symmetric systems. Numerische Mathematik, 94(3):573-602, 2003.

[45] C. Wall, C.D. Pierce, and P. Moin. A semi-implicit method for resolution of acoustic waves in low Mach number flows. J. Comp. Phys., 181:545-563, 2002.

[46] I. Wenneker, A. Segal, and P. Wesseling. A Mach-uniform unstructured staggered grid method. Intern. J. Numer. Method. Fluid., 40:1209-1235, 2002.

[47] P. Wesseling. Principles of computational fluid dynamics, volume 29 of Springer Series in Computational Mathematics. Springer-Verlag, Berlin, 2001.

[48] V Yovanovic. An error estimate for a numerical scheme for the compressible Navier-Stokes system. Kragujevac J. Math, 30(1):263-275, 2007. 